\title{
Copper-Catalyzed Alkoxycarbonylation of Alkyl Iodides for the Synthesis of Aliphatic Esters: Hydrogen Makes the Difference
}

Hui-Qing Geng ${ }^{[a]}$ and Xiao-Feng $\mathrm{Wu}^{*[a, b]}$

${ }^{[a]}$ Leibniz-Institut für Kataly see.V., Albert-Einstein-Straße 29a, 18059 Rostock, Germany

${ }^{[b]}$ Da lian National Laboratory for Clean Energy, Dalian Institute of ChemicalPhy sics, Chinese Academy of Sciences, 116023, Dalian, Lia oning, China

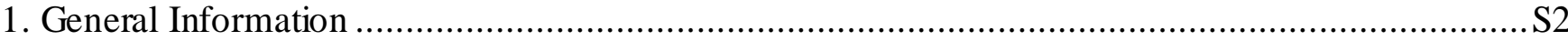

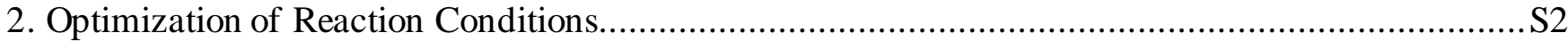

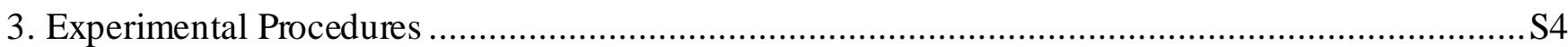

4. General Procedure for Synthesis of Starting Materials......................................................... S5

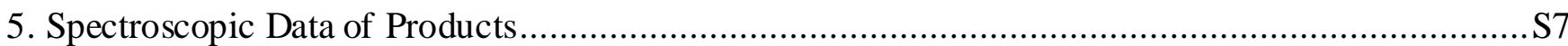

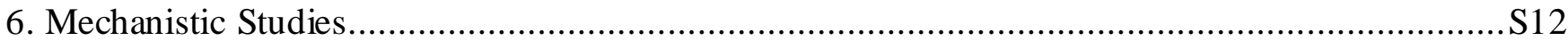

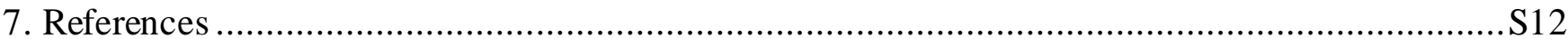

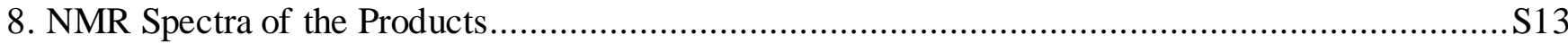




\section{General Information}

Unless otherwise noted, all commercial reagents were ordered from Sigma-Aldrich, TCI, ABCR, or Acros and used as such unless stated otherwise. All solvents (Anhydrous and under inert atmosphere) were collected from the solvent purification system by MBRAUN and used under standard Schlenk technique. Alkyl iodides were synthesized according to the known literature. Column chromatography was performed on silica gel (200-300 meshes) using $n$-pentane (bp. $36.1{ }^{\circ} \mathrm{C}$ ), dichloromethane and ethyl acetate as eluent. All NMR spectra were recorded at ambient temperature using Bruker Advance 300 NMR, Bruker ARX 400 NMR spectrometers. Multiplets were assigned as follows: chemical shift $(\delta \mathrm{ppm})$, multiplicity ( $\mathrm{s}=$ singlet, $\mathrm{d}=$ doublet, $\mathrm{t}=$ triplet, $\mathrm{q}=$ quartet, $\mathrm{m}=$ multiplet, $\mathrm{br}=$ broad), coupling constant $(\mathrm{Hz})$, and integration. All ${ }^{13} \mathrm{C}$ NMR spectra were broad-band ${ }^{1} \mathrm{H}$ decoupled. Gas chromatography (GC) analyses were performed on an Agilent HP-7890A instrument with a FID detector and HP-5 capillary column (polydimethylsiloxane with $5 \%$ phenyl groups, $30 \mathrm{~m}, 0.32 \mathrm{~mm}$ i.d. $0.25 \mu \mathrm{m}$ film thickness) using argon as carrier gas. High resolution mass spectra (HRMS) were recorded on an Agilent 6210 system. Because of the high toxicity of carbon monoxide, all reactions should be performed in an autoclave. The laboratory should be well-equipped with a $\mathrm{CO}$ detector and alarm system.

\section{Optimization of Reaction Conditions}

Table S1. Optimization of Liga nd.

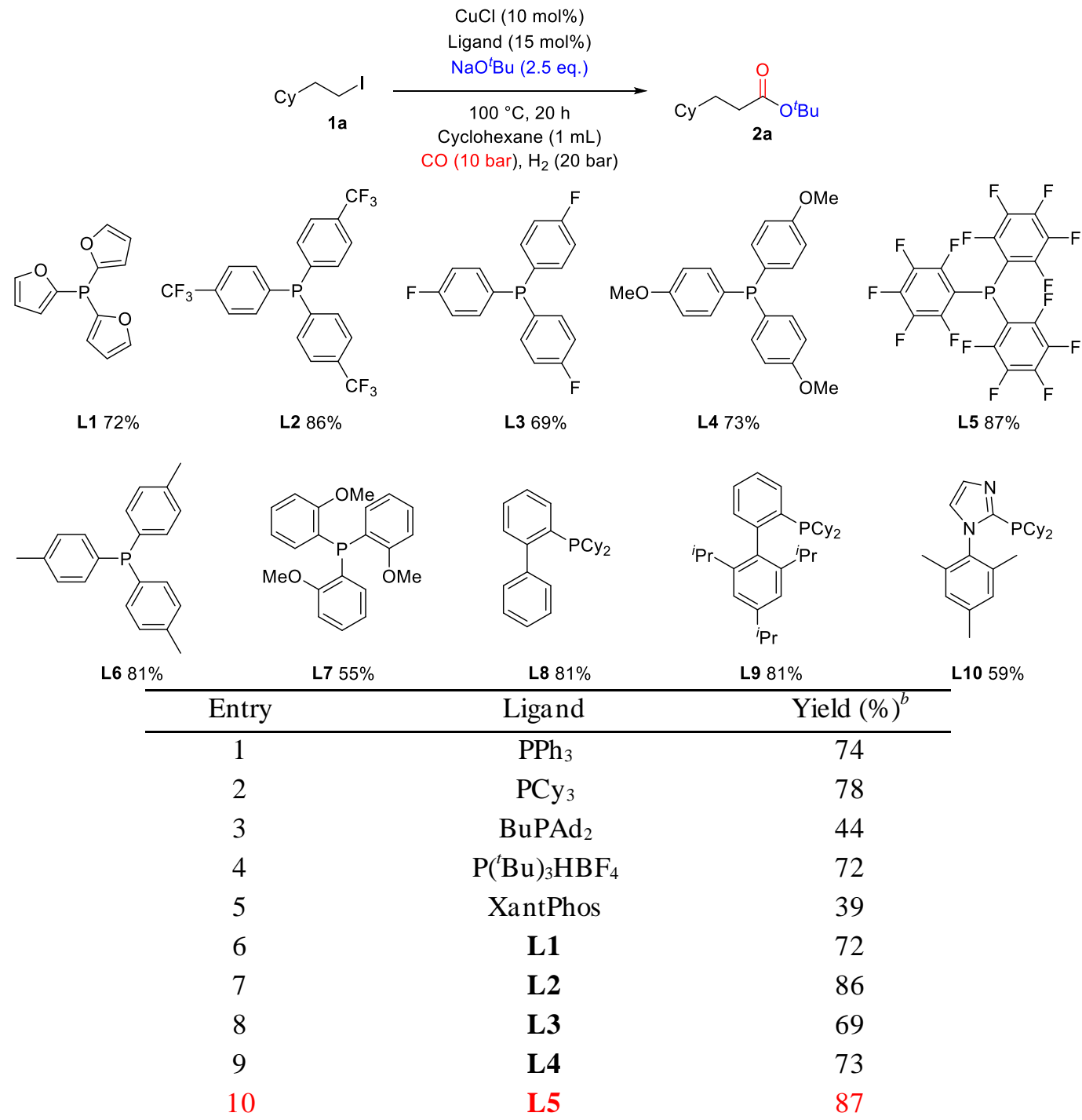




$\begin{array}{lcc}11 & \text { L6 } & 81 \\ 12 & \text { L7 } & 55 \\ 13 & \text { L8 } & 81 \\ 14 & \text { L9 } & 81 \\ 15 & \text { L10 } & 59 \\ 16 & \text { w/o } & 59\end{array}$

${ }^{a}$ Reaction condition: 1a $(0.2 \mathrm{mmol}), \mathrm{CuCl}(10 \mathrm{~mol} \%)$, Ligand $(15$
mol\%), $\mathrm{NaO} \mathrm{O}^{t} \mathrm{Bu}(2.5$ eq. $)$, Cyclohexane (1 mL), $\mathrm{CO}(10 \mathrm{bar}), \mathrm{H}_{2}(20$
bar), $100{ }^{\circ} \mathrm{C}, 20 \mathrm{~h} .{ }^{b} \mathrm{GC}$ yield.

Table S2. Optimization of base.

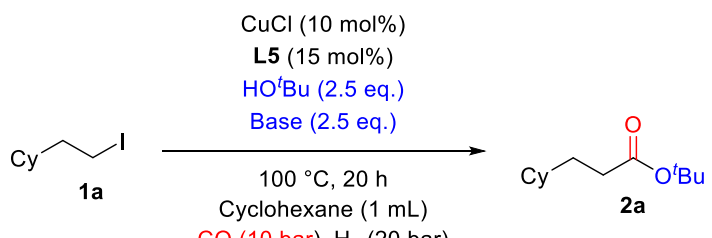

$\mathrm{CO}(10 \mathrm{bar}) \mathrm{H}_{2}(20$ bar $)$

\begin{tabular}{ccc}
\hline Entry & Base (2.5 eq.) & Yield $(\%)^{b}$ \\
\hline 1 & $\mathrm{~K}_{2} \mathrm{CO}_{3}$ & No Reaction \\
2 & $\mathrm{Cs}_{2} \mathrm{CO}_{3}$ & No Reaction \\
3 & $\mathrm{NaOH}$ & No Reaction
\end{tabular}

${ }^{a}$ Reaction condition: 1a $(0.2 \mathrm{mmol}), \mathrm{CuCl}(10 \mathrm{~mol} \%)$, Ligand $(15$ mol\%), Base (2.5 eq.), $\mathrm{NaO}^{\prime} \mathrm{Bu}$ (2.5 eq.), Cyclohexane (1 mL), $\mathrm{CO}(10$ bar), $\mathrm{H}_{2}(20 \mathrm{bar}), 100{ }^{\circ} \mathrm{C}, 20 \mathrm{~h} .{ }^{b} \mathrm{GC}$ yield.

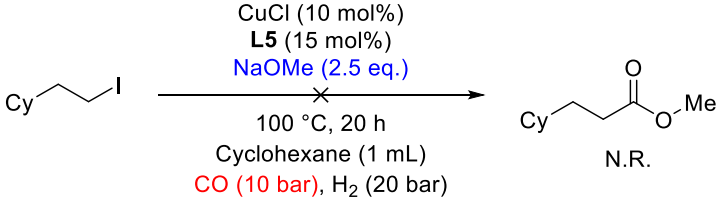

Table S3. Optimization of copper-cataly sis.

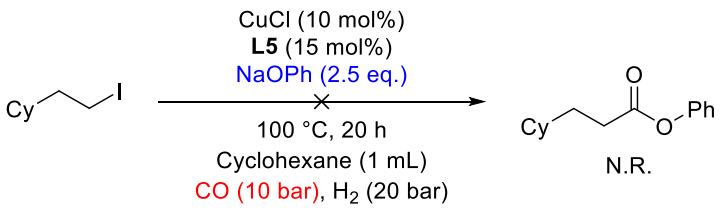

\begin{tabular}{|c|c|c|}
\hline \multirow{2}{*}{ cy } & $\begin{array}{l}{[\mathrm{Cu}](10 \mathrm{~mol} \%)} \\
\mathrm{L} 5(15 \mathrm{~mol} \%) \\
\text { NaOtBu (2.5 eq.) }\end{array}$ & \multirow{2}{*}{ If } \\
\hline & $\begin{array}{c}100^{\circ} \mathrm{C}, 20 \mathrm{~h} \\
\text { Cyclohexane }(1 \mathrm{~mL}) \\
\text { CO }(10 \mathrm{bar}), \mathrm{H}_{2}(20 \mathrm{bar})\end{array}$ & \\
\hline Entry & {$[\mathrm{Cu}]$} & Yield $(\%)^{b}$ \\
\hline 1 & $\mathrm{CuCl}$ & 77 \\
\hline 2 & $\mathrm{CuCl}_{2}$ & 77 \\
\hline 3 & $\mathrm{Cu}(\mathrm{OTf})_{2} \cdot$ Toluene & 61 \\
\hline 4 & $\mathrm{CuI}$ & 68 \\
\hline 5 & $\mathrm{CuBr}$ & 67 \\
\hline 6 & $\mathrm{Cu}(\mathrm{OAc})_{2}$ & 74 \\
\hline 7 & $\mathrm{CuCN}$ & $91\left(87 \%^{c}\right)$ \\
\hline 8 & $\mathrm{CuCN}$ & $67^{d}$ \\
\hline 9 & $\mathrm{Cu}(\mathrm{acac})_{2}$ & 62 \\
\hline 10 & ${ }^{\mathrm{Me}} \mathrm{IPrCuCl}$ & 6 \\
\hline 11 & IMesCuCl & 67 \\
\hline
\end{tabular}




$\begin{array}{lll}12 & \mathrm{CuNO}_{3}\left(\mathrm{PPh}_{3}\right)(1,10-\text { phen }) & 77 \\ 13 & \mathrm{CuBr}\left(\mathrm{PPh}_{3}\right)(1,10-\text { phen }) & 81\end{array}$

${ }^{a}$ Reaction condition: 1a $(0.2 \mathrm{mmol}),[\mathrm{Cu}](10 \mathrm{~mol} \%), \mathbf{L 5}(15 \mathrm{~mol} \%)$,

$\mathrm{NaO}^{t} \mathrm{Bu}$ (2.5 eq.), Cyclohexane (1 mL), CO (10 bar), $\mathrm{H}_{2}$ (20 bar), $100{ }^{\circ} \mathrm{C}, 20 \mathrm{~h}^{b}{ }^{b} \mathrm{GC}$ yield. ${ }^{c}$ Isolated yield. ${ }^{d} 80{ }^{\circ} \mathrm{C}$

Table S4. Optimization of solvent.

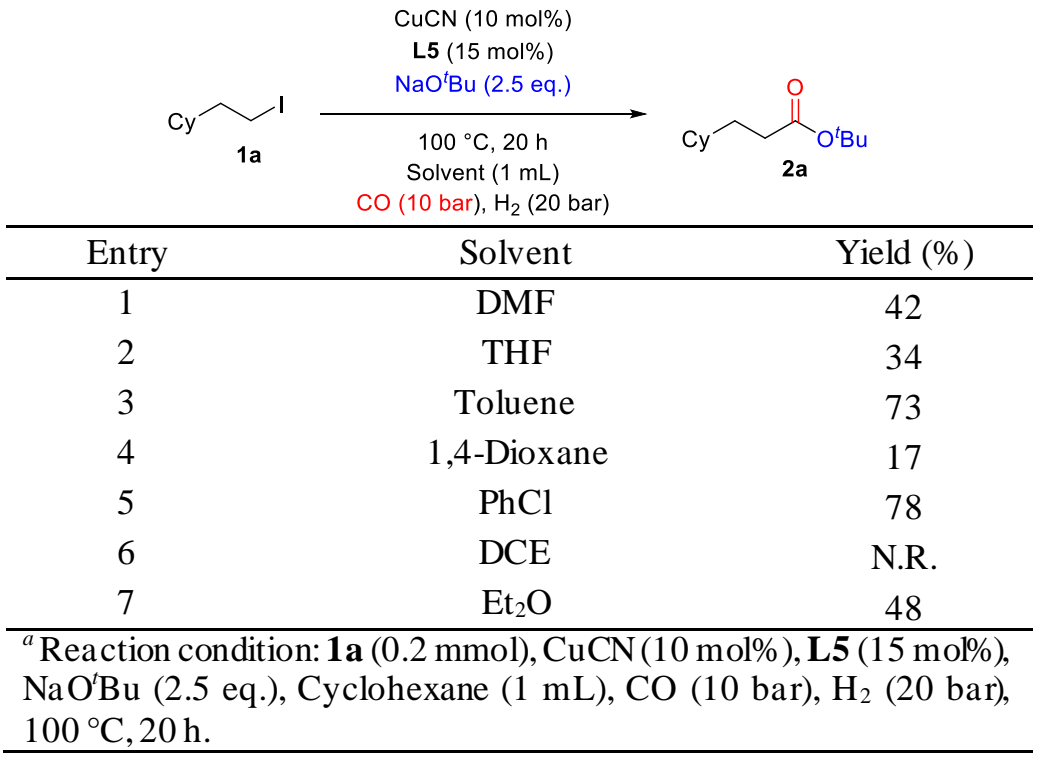

\section{Experimental Procedures}

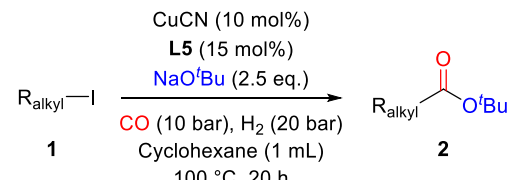

\section{General Procedure A}

A dried $4 \mathrm{~mL}$ screw-cap vial equipped with a septum and a stirring bar was charged with $\mathrm{CuCN}(1.8 \mathrm{mg}, 10 \mathrm{~mol} \%), \mathrm{L} 5$ (15 $\mathrm{mol} \%$ ), and $\mathrm{NaO}^{t} \mathrm{Bu}(48.1 \mathrm{mg}, 2.5$ equiv). The vial wa s sealed, connected to atmosphere with a small cannula, evacuated, and backfilled with argon three times. Cyclohexane $(1.0 \mathrm{~mL})$ were added via syringe. To this su spension, substrate $\mathbf{1}(0.2 \mathrm{mmol})$ was a dded via Hamilton ${ }^{\circledR}$ syringe. The vial was moved to an alloy plate and put into a Parr 4560 series autoclave $(300 \mathrm{~mL})$ under an argon atmosphere. At room temperature, the autoclave was flushed with $\mathrm{CO}$ three times and charged with $\mathrm{CO}$ (10 bar) and subsequent with $\mathrm{H}_{2}(20 \mathrm{bar})$ to reach the desired pressure of $30 \mathrm{bar}$. The a utoclave was placed on a heating plate equipped with a magnetic stirrer and an aluminum block. The reaction mixture was heated to $100^{\circ} \mathrm{C}$ for $20 \mathrm{~h}$. After the reaction wa s complete, the a utoclave was cooled down to room temperature and the pressure wa s released carefully. The solution was filtered through calite and concentrated in vacuo. The crude reaction mixture was purified by flash chromatograph on silica gel and evaporated to give the product 2.

$1 \mathrm{mmol}$ scale: A dried $12 \mathrm{~mL}$ screw-cap vial equipped with a septum and a stirring bar was charged with $\mathrm{CuCN}(10 \mathrm{~mol} \%)$, L5 (15 mol\%), and $\mathrm{NaO}^{t} \mathrm{Bu}$ (2.5 equiv). The vial was sealed, connected to a tmosphere with a small cannula, evacuated, and backfilled with argon three times. Cyclohexane $(5.0 \mathrm{~mL})$ were added via syringe. To this suspension, substrate $1 \mathbf{a}(1 \mathrm{mmol})$ was a dded via Hamilton ${ }^{\circledR}$ syringe. The vial was moved to an alloy plate and put into a Parr 4560 series autoclave ( $\left.300 \mathrm{~mL}\right)$ under an argon atmosphere. At room temperature, the autoclave was flushed with $\mathrm{CO}$ three times and charged with $\mathrm{CO}$ (10 
bar) and subsequent with $\mathrm{H}_{2}(20 \mathrm{bar})$ to reach the desired pressure of $30 \mathrm{bar}$. The a utoclave was placed on a heating plate equipped with a magnetic stirrer and an aluminum block. The reaction mix ture was heated to $100^{\circ} \mathrm{C}$ for $20 \mathrm{~h}$. After the reaction was complete, the a utoclave was cooled down to room temperature and the pressure wa s released carefully. The solution was filtered through calite and concentrated in vacuo. The crude reaction mixture was purified by flash chromatograph on silica gel and evaporated to give the product $\mathbf{2 a}(80 \%$ yield; $169.6 \mathrm{mg})$.

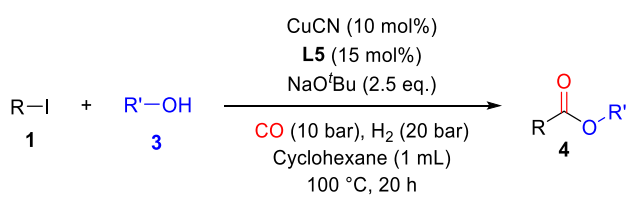

\section{General Procedure B}

A dried $4 \mathrm{~mL}$ screw-cap vialequipped with a septum and a stirring bar was charged with $\mathrm{CuCN}(1.8 \mathrm{mg}, 10 \mathrm{~mol} \%), \mathrm{L} 5$ (15 $\mathrm{mol} \%$ ), and $\mathrm{NaO}^{\prime} \mathrm{Bu}$ (48.1 mg, 2.5 equiv). The vial wa s sealed, connected to atmosphere with a small cannula, evacuated, and backfilled with argon three times. Cyclohexane $(1.0 \mathrm{~mL})$ were added via syringe. To this suspension, substrate $\mathbf{1}(0.2 \mathrm{mmol})$ and a dditional alcohol 3 was a dded via Hamilton ${ }^{\circledR}$ syringe. The vial was moved to an alloy plate and put into a Parr 4560 series autoclave $(300 \mathrm{~mL})$ under an argon atmosphere. At room temperature, the a utoclave was flushed with CO three times and charged with $\mathrm{CO}(10 \mathrm{bar})$ and subsequent with $\mathrm{H}_{2}(20 \mathrm{bar})$ to reach the desired pressure of $30 \mathrm{bar}$. The autoclave was placed on a heating plate equipped with a magnetic stirrer and a n aluminum block. The reaction mixture wa sheated to $100{ }^{\circ} \mathrm{C}$ for $20 \mathrm{~h}$. After the reaction was complete, the a utoclave was cooled down to room temperature and the pressure was released ca refully. The solution was filtered through calite and concentrated in vacuo. Thecrude reaction mixture was purified by flash chromatograph on silica gel and evaporated to give the product 4.

\section{General Procedure for Synthesis of Starting Materials.}

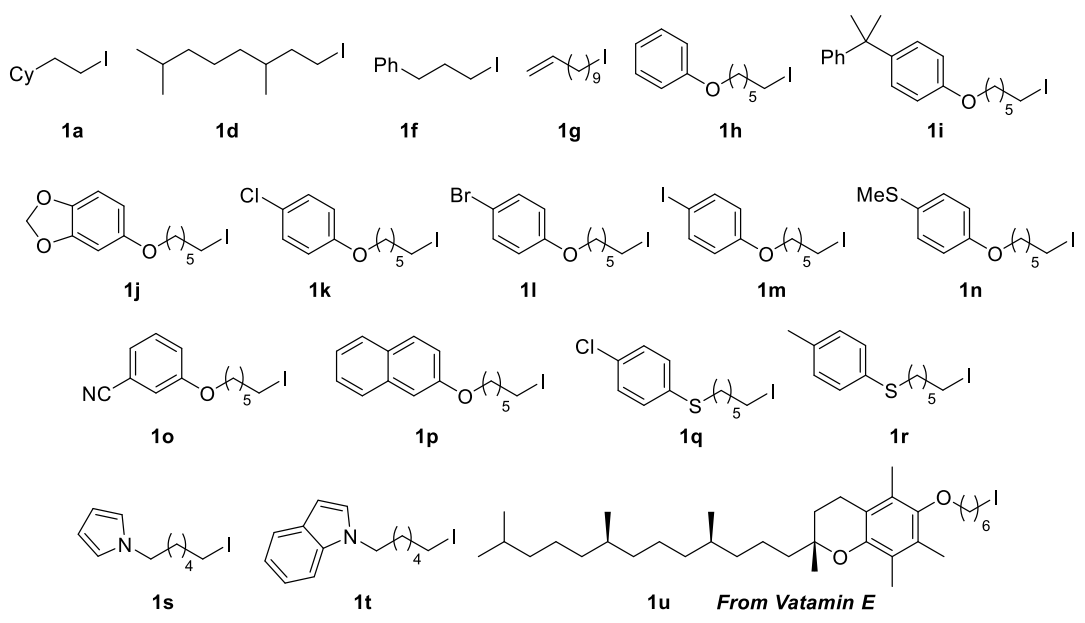

All of the starting materials were produced from the known literatures. 1a, $\mathbf{1 f}$ and $\mathbf{1 g}$ were prepared following the General Procedure C. ${ }^{[1]} 1 \mathrm{~d}$ wa s prepared following the General Procedure D. ${ }^{[1]} \mathbf{1 h}-\mathbf{1} \mathbf{p}$, and $\mathbf{1}$ u were prepared following the General Procedure E. ${ }^{[2]} \mathbf{1 q}$ and $1 \mathbf{r}$ were produced through General ProcedureF that was a dapted from the general procedure reported ${ }^{[3]} \mathbf{1 s}$ and $\mathbf{1 t}$ were syntheses through General Procedure $\mathbf{G}$ a ccording to the known litera tures. ${ }^{[4]}$

\section{General Procedure C}

$$
\mathrm{R}^{\prime} \widehat{\mathrm{OH}} \frac{\mathrm{I}_{2}(1.2 \text { equiv. }}{\mathrm{PPh}_{3}(1.2 \text { equiv. })} \underset{\begin{array}{c}
\text { imidazole }(1.2 \text { equiv. }) \\
\text { DCM, } \mathrm{rt}
\end{array}}{\longrightarrow} \mathrm{R}^{\prime} \widehat{\mathrm{I}}_{\mathrm{I}}
$$

A flame-dried flask with Teflon stir bar was charged with triphenylphosphine (1.2 equiv.) and $\mathrm{I}_{2}$ (1.2 equiv.) in DCM, the mixture was stirred for $10 \mathrm{~min}$ at room temperature. Imidazole (1.2 equiv.) was added to the resulting mix ture. After $10 \mathrm{~min}$ of stirring, the corresponding a lcohol (1.0 equiv.) was added and the reaction was stirred for additional $5 \mathrm{~h}$ at room temperature 
and was checked by TLC until completion. The reaction mix ture was quenched with $\mathrm{Sat}_{2} \mathrm{Na}_{2} \mathrm{SO}_{3}$. The aqueous and organic layers were separated and the aqueous layer wa s extracted by DCM $(3 \mathrm{x})$. The combined organic layers were washed with brine and dried over $\mathrm{Na}_{2} \mathrm{SO}_{4}$, filtered, and concentrated in vacuum. The crude reaction mixture was purified by fla sh chromatograph on silica gel and evaporated to give the alkyl iodide compound.

$$
\mathrm{R}^{\prime} \widehat{\mathrm{Br}} \underset{\text { acetone, reflux }}{\stackrel{\text { Nal (3 equiv.) }}{\longrightarrow}} \mathrm{R}^{\prime} \widehat{\mathrm{I}}
$$

\section{General Procedure D}

A flame-dried flask with Teflon stir bar was charged with alkyl bromide (1.0 equiv.) and NaI (3.0 equiv.) in acetone. The mixture was heated to reflux for $6 \mathrm{~h}$ and was checked by TLC until completion. After being a llowed to cool down to rom temperature, the reaction mixture was quenched with $\mathrm{Sat}_{2} \mathrm{Na}_{2} \mathrm{SO}_{3}$. The aqueous and organic layers were separated, and the aqueous la yer was extracted by DCM (3x). The combined organic layers were washed with brine and dried over $\mathrm{Na}_{2} \mathrm{SO}_{4}$, filtered, and concentrated in vacuum. The crude reaction mixture was purified by flash chromatograph on silica gel and eva porated to give the alkyl iodide compound.

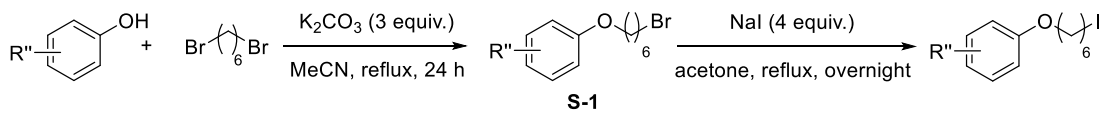

\section{General Procedure E}

A fla me-dried flask with Teflon stir bar wa scharged with the solution of phenol and 1,6-dibromopropane (5 equiv.) in MeCN. Then added $\mathrm{K}_{2} \mathrm{CO}_{3}$ (3 equiv.). The reaction mixture was stirred at reflux for $24 \mathrm{~h}$, and then potassium carbonate was removed by suction filtration and the solvent was removed under reduced pressure. The residue was purified by column chromatography (silica gel) using PE/EtOAc a seluent to obtain compound S-1. For iodination step, NaI (10 eq.) were dissolved in a cetone, and the crude mixture obtained from last step was added to the solution slowly. The mix ture was heated to reflux for $6 \mathrm{~h}$ and was checked by TLC until completion. After being a llowed to cool down to room temperature, the reaction mixture was quenched with Sat. $\mathrm{Na}_{2} \mathrm{SO}_{3}$. The a queous and organic la yers were separated, and the aqueous la yer was extracted by DCM (3x). The combined organic layers were washed with brine and dried over $\mathrm{Na}_{2} \mathrm{SO}_{4}$, filtered, and concentrated in vacuum. The crude reaction mix ture was purified by flash chromatograph on silica gel and evaporated to give the alkyl iodide compound.

$$
\text { R'" }
$$

\section{General Procedure F}

To a stirred solution of $\mathrm{NaH}$ (2 equiv.) in DMF at $0{ }^{\circ} \mathrm{C}$ was a dded Thiophenol. After $90 \mathrm{~min}$, the solution wastransferred into another flask containing a solution of 1,6-dibromopropane (5 equiv.) in DMF at $0{ }^{\circ} \mathrm{C}$. The reaction mixture was allowed to warm to room temperature and stirred at room temperature for $20 \mathrm{~h}$. Workup the reaction by adding $50 \mathrm{~mL}$ wa ter and $50 \mathrm{~mL}$ EtOAc to the reaction mix ture. The orga nic layer was wa shed with water $(5 \times 50 \mathrm{~mL})$ in a sepa ratory funnel then collected, dried over $\mathrm{Na}_{2} \mathrm{SO}_{4}$. Solvent wa s removed by a rotatory evaporator. And purified by column chromatography (silica gel) u sing $\mathrm{PE} /$ EtOAc as eluent to obtain compound S-2. For iodination step, $\mathrm{NaI}$ (10 eq.) were dissolved in acetone, and the crude mixture obtained from last step was added to the solution slowly. The mixture was heated to reflux for $6 \mathrm{~h}$ and was checked by TLC until completion. After being a llowed to cool down to room temperature, the reaction mix ture was quenched with $\mathrm{Sat} \mathrm{Na}_{2} \mathrm{SO}_{3}$. The aqueous and orga nic layers were separated, and the aqueous layer was extracted by DCM (3x). The combined organic layers were washed with brine and dried over $\mathrm{Na}_{2} \mathrm{SO}_{4}$, filtered, and concentrated in vacuum. The crude reaction mixture was purified by fla sh chromatograph on silica gel and evaporated to give the alkyliodide compound. 


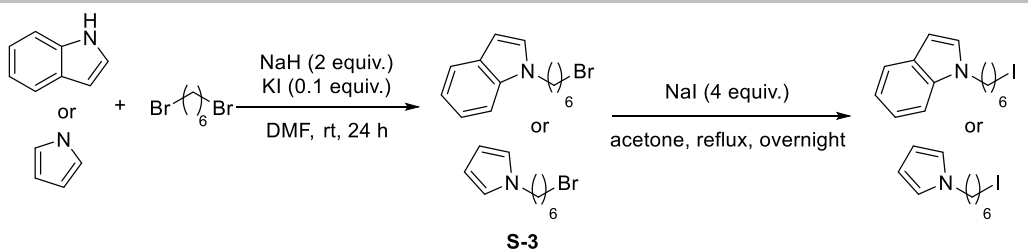

\section{General Procedure G}

Indole or furan (1.0 equiv.) was dissolved with dimethylformamide(DMF) in a round bottom charged with stir bar. Then NaH (2 equiv.) was added to the mix ture, stirring for half an hour at room temperature. 1,6-dibromohexane (5.0 equiv.) and KI (0.1 equiv.) were added. The mixture was left stirring overnight a troom temperature. Workup the reaction by a dding $50 \mathrm{~mL}$ water and $50 \mathrm{mLEtOAc}$ to the reaction mixture. The organic la yer was wa shed with water $(5 \times 50 \mathrm{~mL})$ in a separatory funnel then collected, dried over $\mathrm{Na}_{2} \mathrm{SO}_{4}$. Solvent was removed by a rotatory evaporator. Compound $\mathbf{S - 3}$ was purified by column chromatography (silica gel) using PE/EtOAc. For iodination step, $\mathrm{NaI}$ (10 eq.) were dissolved in acetone, and the crude mixture obtained from last step was added to the solution slowly. The mixture was heated to reflux for $6 \mathrm{~h}$ and was checked by TLC until completion. After being a llowed to cool down to room temperature, the reaction mixture was quenched with $\mathrm{Sat} \mathrm{Na}_{2} \mathrm{SO}_{3}$. The aqueous and orga nic layers were separated, and the aqueous layer was extracted by DCM (3x). The combined organic layers were washed with brine and dried over $\mathrm{Na}_{2} \mathrm{SO}_{4}$, filtered, and concentrated in vacuum. The crude reaction mixture was purified by fla sh chromatograph on silica gel and evaporated to give the alkyliodide compound.

\section{Spectroscopic Data of Products}

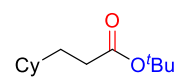

tert-butyl3-cyclohexylpropanoate $(\mathbf{2 a})^{[5]}$

The title compound was prepared from (2-iodoethyl)cy clohexane ( $47.6 \mathrm{mg}, 0.2 \mathrm{mmol}$ ), according to General Procedure A. The crude residue was purified by flash chromatography (pentane/EA $=100: 1, \mathrm{Rf}=0.30$ ) to give the product as a colorless oil $(36.9 \mathrm{mg}, 87 \%)$.

${ }^{1} \mathbf{H}$ NMR $(300 \mathrm{MHz}$, Chloroform- $d) \delta 2.20(\mathrm{t}, J=7.9 \mathrm{~Hz}, 2 \mathrm{H}), 1.70-1.64(\mathrm{~m}, 5 \mathrm{H}), 1.49-1.44(\mathrm{~m}, 2 \mathrm{H}), 1.42(\mathrm{~s}, 9 \mathrm{H}), 1.22-$ $1.14(\mathrm{~m}, 4 \mathrm{H}), 0.92-0.84(\mathrm{~m}, 2 \mathrm{H}) .{ }^{13} \mathbf{C N M R}\left(75 \mathrm{MHz}, \mathrm{CDCl}_{3}\right) \delta 173.6,79.8,37.2,33.2,33.0,32.4,28.1,26.5,26.2$.

$$
\text { Cy } \overbrace{\text { OEt }}
$$

ethyl3-cyclohexylpropanoate $(\mathbf{2 b})^{[6]}$

The title compound was prepared from (2-iodoethyl)cyclohexane $(47.6 \mathrm{mg}, 0.2 \mathrm{mmol}$ ) and $\mathrm{NaOEt}(34 \mathrm{mg}, 2.5 \mathrm{eq}$ ), a ccording to General Procedure A. The crude residue was purified by fla sh chromatography (pentane/EA $=100: 1, \mathrm{Rf}=0.30$ ) to give the product as a colorless oil $(27.6 \mathrm{mg}, 75 \%)$.

${ }^{1} \mathbf{H}$ NMR $(300 \mathrm{MHz}$, Chloroform- $d) \delta 4.12(\mathrm{q}, J=7.1 \mathrm{~Hz}, 2 \mathrm{H}), 2.30(\mathrm{t}, J=7.9 \mathrm{~Hz}, 2 \mathrm{H}), 1.72-1.66(\mathrm{~m}, 4 \mathrm{H}), 1.52-1.41(\mathrm{~m}$, $3 \mathrm{H}), 1.24(\mathrm{t}, J=7.1 \mathrm{~Hz}, 3 \mathrm{H}), 1.23-1.02(\mathrm{~m}, 4 \mathrm{H}), 0.93-0.83(\mathrm{~m}, 2 \mathrm{H}) .{ }^{13} \mathbf{C N M R}\left(75 \mathrm{MHz}, \mathrm{CDCl}_{3}\right) \delta$ 174.2, 60.2, 37.2, 33.0, $32.4,32.0,26.5,26.2,14.2$.

$\mathrm{Cy}_{\mathrm{O}}^{\mathrm{t} B \mathrm{Bu}}$

tert-butyl2-cyclohexylacetate (2c)

The title compound wa s prepared from (iodomethyl)cy clohexane (44.8 mg, $0.2 \mathrm{mmol}$ ), a ccording to General ProcedureA. The crude residue was purified by flash chromatography (pentane/EA $=100: 1, \mathrm{Rf}=0.30$ ) to give the product as a colorless oil (36.8 mg, 93\%).

${ }^{1}$ H NMR (300 MHz, Chloroform- $d$ ) $\delta 2.08(\mathrm{~d}, J=6.7 \mathrm{~Hz}, 2 \mathrm{H}), 1.77-1.60(\mathrm{~m}, 6 \mathrm{H}), 1.44(\mathrm{~s}, 9 \mathrm{H}), 1.30-1.12(\mathrm{~m}, 3 \mathrm{H}), 1.01-$ $0.87(\mathrm{~m}, 2 \mathrm{H}) .{ }^{13} \mathbf{C ~ N M R}\left(75 \mathrm{MHz}, \mathrm{CDCl}_{3}\right) \delta 172.6,79.9,43.5,35.0,33.0,28.1,26.2,26.1$.<smiles>CC(C)CCCOC(=O)CCC(C)CCCC(C)C</smiles>

tert-butyl4,8-dimethylnonanoate (2d)

The title compound wa s prepared from 1-iodo-3,7-dimethyloctane (53.6 mg, $0.2 \mathrm{mmol}$ ), a ccording to General Procedure A. The crude residue was purified by flash chromatography (pentane/EA $=100: 1, \mathrm{Rf}=0.30$ ) to give the product as a colorless oil (47.5 mg, 98\%). 
${ }^{1} \mathbf{H}$ NMR $(300 \mathrm{MHz}$, Chloroform- $d$ ) $\delta 2.19(\mathrm{t}, J=7.7 \mathrm{~Hz}, 2 \mathrm{H}), 1.65-1.51(\mathrm{~m}, 2 \mathrm{H}), 1.44(\mathrm{~s}, 9 \mathrm{H}), 1.42-1.39(\mathrm{~m}, 2 \mathrm{H}), 1.28-$ $1.07(\mathrm{~m}, 6 \mathrm{H}), 0.86(\mathrm{~d}, J=6.6 \mathrm{~Hz}, 9 \mathrm{H}) .{ }^{13} \mathbf{C N M R}\left(75 \mathrm{MHz}, \mathrm{CDCl}_{3}\right) \delta 173.6,79.9,39.2,36.9,33.4,32.4,32.1,28.1,24.7,22.7$, 22.6, 19.3.

\section{$\sim^{\text {Ill Bu }}$}

tert-butylheptanoate $(\mathbf{2 e})^{[7]}$

The title compound was prepared from 1 -iodohexane $(42.4 \mathrm{mg}, 0.2 \mathrm{mmol})$, according to General Procedure A. The crude residue was purified by flash chromatography (pentane $/ E A=100: 1, R f=0.30$ ) to give the product as a colorless oil $(34.3 \mathrm{mg}$, $92 \%)$.

${ }^{1} \mathbf{H}$ NMR $(300 \mathrm{MHz}$, Chloroform- $d) \delta 2.20(\mathrm{t}, J=7.5 \mathrm{~Hz}, 2 \mathrm{H}), 1.64-1.50(\mathrm{~m}, 2 \mathrm{H}), 1.44(\mathrm{~s}, 9 \mathrm{H}), 1.42-1.42(\mathrm{~m}, 6 \mathrm{H}), 0.89(\mathrm{t}$, $J=6.9 \mathrm{~Hz}, 3 \mathrm{H}) .{ }^{13} \mathbf{C} \mathbf{N M R}\left(75 \mathrm{MHz}, \mathrm{CDCl}_{3}\right) \delta 173.4,79.9,35.6,31.5,28.8,28.1,25.1,22.5,14.0$. HRMS (EI): calcd for [M] $]^{+}$ $\mathrm{C}_{11} \mathrm{H}_{22} \mathrm{O}_{2}$ 186.1614, found: 186.1608 .

$\mathrm{Nh}_{\mathrm{O} B \mathrm{Bu}}^{\mathrm{O}}$

tert-butyl4-phenylbutanoate $(\mathbf{2 f})^{[8]}$

The title compound was prepared from (3-iodopropyl)benzene $(49.2 \mathrm{mg}, 0.2 \mathrm{mmol}$ ), according to General Procedure A. The crude residue was purified by flash chromatography (pentane/EA $=100: 1, \mathrm{Rf}=0.30$ ) to give the product as a colorless oil (28.2 $\mathrm{mg}, 64 \%)$.

${ }^{1} \mathrm{H}$ NMR (300 MHz, Chloroform- $d$ ) $87.37-7.28(\mathrm{~m}, 2 \mathrm{H}), 7.27-7.16(\mathrm{~m}, 3 \mathrm{H}), 2.67(\mathrm{t}, J=7.6 \mathrm{~Hz}, 2 \mathrm{H}), 2.27(\mathrm{t}, J=7.5 \mathrm{~Hz}$, $2 \mathrm{H}), 1.99-1.89(\mathrm{~m}, 2 \mathrm{H}), 1.48(\mathrm{~s}, 9 \mathrm{H}) .{ }^{13} \mathbf{C} \mathbf{N M R}\left(75 \mathrm{MHz}, \mathrm{CDCl}_{3}\right) \delta$ 172.9, $141.6,128.5,128.3,125.9,80.1,35.1,34.9,28.1$, 26.7.

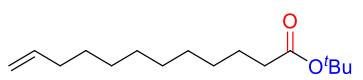

tert-butyldodec-11-enoate (2g)

The title compound was prepared from 11-iodoundec-1-ene (56.0 mg, $0.2 \mathrm{mmol})$, according to General Procedure A. The crude residue was purified by flash chromatography (pentane/EA $=100: 1, \mathrm{Rf}=0.30$ ) to give the product as a colorless oil (36.6 mg, 72\%).

${ }^{1}$ H NMR $(300 \mathrm{MHz}$, Chloroform- $d) \delta 5.88-5.74(\mathrm{~m}, 1 \mathrm{H}), 5.03-4.89(\mathrm{~m}, 2 \mathrm{H}), 2.19(\mathrm{t}, J=7.5 \mathrm{~Hz}, 2 \mathrm{H}), 2.07-1.99(\mathrm{~m}, 2 \mathrm{H})$, $1.59-1.54(\mathrm{~m}, 2 \mathrm{H}), 1.44(\mathrm{~s}, 9 \mathrm{H}), 1.37-1.27(\mathrm{~m}, 12 \mathrm{H}){ }^{13} \mathbf{C} \mathbf{N M R}\left(75 \mathrm{MHz}, \mathrm{CDCl}_{3}\right) \delta$ 173.3, 139.2, 114.1, 79.9, 35.6, 33.8, 29.4, 29.3,29.1, 29.1, 28.9, 28.4, 28.1, 25.1. HRMS (EI): calcd for [M] ${ }^{+} \mathrm{C}_{16} \mathrm{H}_{30} \mathrm{O}_{2}$ 254.2240, found:254.2240.

\section{Sor}

tert-butyl 7-phenoxyheptanoate (2h)

The title compound was prepared from ((6-iodohexyl)oxy)benzene ( $60.8 \mathrm{mg}, 0.2 \mathrm{mmol})$, a ccording to General Procedure A. The crude residue was purified by flash chromatography (pentane/EA $=100: 1, \mathrm{Rf}=0.30$ ) to give the product as a colorless oil (42.8 mg, 77\%).

${ }^{1} \mathbf{H}$ NMR $(300 \mathrm{MHz}$, Chloroform- $d$ ) $\delta 7.30-7.26(\mathrm{~m}, 2 \mathrm{H}), 6.95-6.87(\mathrm{~m}, 3 \mathrm{H}), 3.95(\mathrm{t}, J=6.5 \mathrm{~Hz}, 2 \mathrm{H}), 2.22(\mathrm{t}, J=7.4 \mathrm{~Hz}$, $2 \mathrm{H}), 1.83-1.75(\mathrm{~m}, 2 \mathrm{H}), 1.68-1.58(\mathrm{~m}, 2 \mathrm{H}), 1.55-1.46(\mathrm{~m}, 2 \mathrm{H}), 1.44(\mathrm{~s}, 9 \mathrm{H}), 1.42-1.34(\mathrm{~m}, 2 \mathrm{H}) .{ }^{13} \mathbf{C} \mathbf{N M R}(75 \mathrm{MHz}$, $\left.\mathrm{CDCl}_{3}\right) \delta 173.2,159.1,129.4,120.5,114.5,80.0,67.7,35.5,29.1,28.8,28.1,25.8,25.0$. HRMS (EI): calcd for $[\mathrm{M}]^{+} \mathrm{C}_{17} \mathrm{H}_{20} \mathrm{O}_{3}$ 278.1876, found: 278.1876.<smiles>COC(=O)CCCCCCOc1ccc(C(C)(C)c2ccccc2)cc1</smiles>

tert-buty17-(4-(2-phenylpropan-2-yl)phenoxy)heptanoate (2i)

The title compound was prepared from 1-((6-iodohexyl)oxy)-4-(2-phenylpropan-2-yl)benzene ( $84.4 \mathrm{mg}, 0.2 \mathrm{mmol})$, according to General Procedure A. The crude residue was purified by fla sh chromatography (pentane/EA $=100: 1, \mathrm{Rf}=0.30$ ) to give the product as a colorless oil $(64.2 \mathrm{mg}, 81 \%)$.

${ }^{1} \mathbf{H}$ NMR $(400 \mathrm{MHz}$, Chloroform- $d$ ) $\delta 7.29-7.11(\mathrm{~m}, 5 \mathrm{H}), 7.05(\mathrm{~d}, J=8.9 \mathrm{~Hz}, 2 \mathrm{H}), 6.71(\mathrm{~d}, J=8.9 \mathrm{~Hz}, 2 \mathrm{H}), 3.85(\mathrm{t}, J=6.5$ $\mathrm{Hz}, 2 \mathrm{H}), 2.14(\mathrm{t}, J=7.5 \mathrm{~Hz}, 2 \mathrm{H}), 1.75-1.67(\mathrm{~m}, 2 \mathrm{H}), 1.58(\mathrm{~s}, 6 \mathrm{H}), 1.56-1.50(\mathrm{~m}, 2 \mathrm{H}), 1.37(\mathrm{~s}, 9 \mathrm{H}), 1.35-1.26(\mathrm{~m}, 4 \mathrm{H})$. ${ }^{13} \mathbf{C}$ NMR $\left(101 \mathrm{MHz}, \mathrm{CDCl}_{3}\right) \delta 173.2,156.9,151.0,142.7,127.9,127.7,126.7,125.5,113.8,80.0,67.7,42.3,35.5,30.9,29.2$, 28.8, 28.1,25.8, 25.0. HRMS (EI): calcd for $[\mathrm{M}]^{+} \mathrm{C}_{26} \mathrm{H}_{36} \mathrm{O}_{3} 396.2659$, found: 396.2651 .<smiles>COC(=O)CCCCCCOc1ccc2c(c1)OCO2</smiles>

tert-butyl7-(benzo[ $d][1,3]$ diox ol-5-yloxy)heptanoate (2j)

The title compound was prepared from 5-((6-iodohexyl)oxy)benzo[ $d][1,3]$ dioxole $(69.6 \mathrm{mg}, 0.2 \mathrm{mmol})$, a ccording to General Procedure A. The crude residue was purified by fla sh chromatography (pentane/EA $=30: 1, R f=0.30$ ) to give the product as a colorless oil $(41.9 \mathrm{mg}, 65 \%)$. 
${ }^{1} \mathbf{H}$ NMR $(400 \mathrm{MHz}$, Chloroform- $d) \delta 6.69(\mathrm{~d}, J=8.5 \mathrm{~Hz}, 1 \mathrm{H}), 6.48(\mathrm{~d}, J=2.5 \mathrm{~Hz}, 1 \mathrm{H}), 6.30(\mathrm{dd}, J=8.5,2.5 \mathrm{~Hz}, 1 \mathrm{H}), 5.90$ $(\mathrm{s}, 2 \mathrm{H}), 3.87(\mathrm{t}, J=6.5 \mathrm{~Hz}, 2 \mathrm{H}), 2.22(\mathrm{t}, J=7.5 \mathrm{~Hz}, 2 \mathrm{H}), 1.77-1.71(\mathrm{~m}, 2 \mathrm{H}), 1.67-1.59(\mathrm{~m}, 2 \mathrm{H}), 1.44(\mathrm{~s}, 9 \mathrm{H}), 1.41-1.31$ $(\mathrm{m}, 4 \mathrm{H}) .{ }^{13} \mathrm{C} \mathrm{NMR}\left(101 \mathrm{MHz}, \mathrm{CDCl}_{3}\right) \delta 173.2,154.6,148.2,141.4,107.9,105.6,101.0,98.0,80.0,68.8,35.5,29.1,28.8$, 28.1, 25.7, 25.0. HRMS (EI): calcd for $[\mathrm{M}]^{+} \mathrm{C}_{18} \mathrm{H}_{26} \mathrm{O}_{5} 322.1775$, found: 322.1772 .

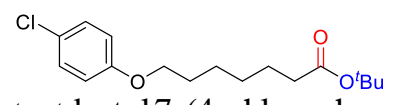

tert-butyl 7-(4-chlorophenoxy)heptanoate (2k)

The title compound was prepared from 1-chloro-4-((6-iodohexyl)oxy)benzene (67.6 mg, $0.2 \mathrm{mmol})$, according to General Procedure A. The crude residue was purified by fla sh chromatography (pentane/EA =100:1, $\mathrm{Rf}=0.30$ ) to give the product as a colorless oil $(51.8 \mathrm{mg}, 83 \%)$.

${ }^{1} \mathrm{H}$ NMR $(400 \mathrm{MHz}$, Chloroform- $d) \delta 7.21(\mathrm{~d}, J=9.1 \mathrm{~Hz}, 2 \mathrm{H}), 6.80(\mathrm{~d}, J=9.0 \mathrm{~Hz}, 2 \mathrm{H}), 3.91(\mathrm{t}, J=6.5 \mathrm{~Hz}, 2 \mathrm{H}), 2.22(\mathrm{t}, J=$ $7.5 \mathrm{~Hz}, 2 \mathrm{H}), 1.79-1.73(\mathrm{~m}, 2 \mathrm{H}), 1.65-1.59(\mathrm{~m}, 2 \mathrm{H}), 1.44(\mathrm{~s}, 9 \mathrm{H}), 1.42-1.25(\mathrm{~m}, 4 \mathrm{H}) .{ }^{13} \mathbf{C N M R}\left(101 \mathrm{MHz}, \mathrm{CDCl}_{3}\right) \delta 173.1$, 157.7, 129.2, 125.3, 115.7, 80.0, 68.1, 35.4, 29.0, 28.8, 28.1, 25.7, 24.9. HRMS (EI): calcd for $[\mathrm{M}]^{+} \mathrm{C}_{17} \mathrm{H}_{25} \mathrm{O}_{3} \mathrm{Cl} 312.1487$, found: 312.1488 .

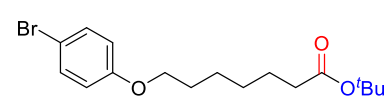

tert-butyl 7-(4-bromophenoxy)heptanoate (2I)

The title compound was prepared from 1-bromo-4-((6-iodohexyl)oxy)benzene (76.4 mg, $0.2 \mathrm{mmol})$, according to General Procedure A. The crude residue was purified by fla sh chromatography (pentane/EA $=100: 1, \mathrm{Rf}=0.30$ ) to give the product as a colorless oil (56.3 $\mathrm{mg}, 79 \%)$.

${ }^{1} \mathbf{H}$ NMR $(400 \mathrm{MHz}$, Chloroform- $d) \delta 7.35(\mathrm{~d}, J=8.1 \mathrm{~Hz}, 2 \mathrm{H}), 6.76(\mathrm{~d}, J=8.2 \mathrm{~Hz}, 2 \mathrm{H}), 3.91(\mathrm{t}, J=6.4 \mathrm{~Hz}, 2 \mathrm{H}), 2.22(\mathrm{t}, J=$ $7.4 \mathrm{~Hz}, 2 \mathrm{H}), 1.80-1.75(\mathrm{~m}, 2 \mathrm{H}), 1.65-1.58(\mathrm{~m}, 2 \mathrm{H}), 1.44(\mathrm{~s}, 9 \mathrm{H}), 1.43-1.42(\mathrm{~m}, 4 \mathrm{H}) .{ }^{13} \mathbf{C N M R}\left(101 \mathrm{MHz}, \mathrm{CDCl}_{3}\right) \delta 173.2$, 158.2, 132.2, 116.3, 86.1, 80.0, 68.1, 35.5, 29.0, 28.8, 28.1, 25.7, 25.0. HRMS (EI): calcd for $[\mathrm{M}]^{+} \mathrm{C}_{17} \mathrm{H}_{25} \mathrm{O}_{3} \mathrm{Br} 356.0982$, found: 356.0975 .

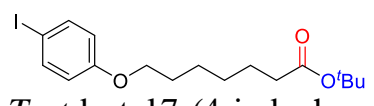

Tert-butyl 7-(4-iodophenoxy)heptanoate (2m)

The title compound was prepared from 1-iodo-4-((6-iodohexyl)oxy)benzene ( $86.0 \mathrm{mg}, 0.2 \mathrm{mmol})$, according to General Procedure A. The crude residue was purified by flash chromatography (pentane/EA $=100: 1, \mathrm{Rf}=0.30$ ) to give the product as a colorless oil $(59.8 \mathrm{mg}, 74 \%)$.

${ }^{1} \mathrm{H}$ NMR $(300 \mathrm{MHz}$, Chloroform- $d) \delta 7.53(\mathrm{~d}, J=9.0 \mathrm{~Hz}, 2 \mathrm{H}), 6.66(\mathrm{~d}, J=9.0 \mathrm{~Hz}, 2 \mathrm{H}), 3.90(\mathrm{t}, J=6.5 \mathrm{~Hz}, 2 \mathrm{H}), 2.22(\mathrm{t}, J=$ $7.4 \mathrm{~Hz}, 2 \mathrm{H}), 1.81-1.71(\mathrm{~m}, 2 \mathrm{H}), 1.65-1.57(\mathrm{~m}, 2 \mathrm{H}), 1.44(\mathrm{~s}, 9 \mathrm{H}), 1.41-1.26(\mathrm{~m}, 4 \mathrm{H}) .{ }^{13} \mathbf{C N M R}\left(75 \mathrm{MHz}, \mathrm{CDCl}_{3}\right) \delta 173.1$, $158.9,138.1,116.9,82.4,80.0,67.9,35.5,28.9,28.8,28.1,25.7,25.0$. HRMS (EI): calcd for $[\mathrm{M}]^{+} \mathrm{C}_{17} \mathrm{H}_{25} \mathrm{O}_{3} \mathrm{I} 404.0843$, found: 404.0832.<smiles>COC(O)CCCCCCOc1ccc(S(C)(=O)=O)cc1</smiles>

tert-butyl 7-(4-(methylthio)phenoxy)heptanoate (2n)

The title compound was prepared from (4-((6-iodohexyl)oxy)phenyl)(methyl)sulfane (70.0 mg, $0.2 \mathrm{mmol})$, according to General Procedure A. The crude residue was purified by flash chromatography (pentane/EA $=100: 1, \mathrm{Rf}=0.30$ ) to give the product as a colorless oil $(40.8 \mathrm{mg}, 63 \%)$.

${ }^{1} \mathrm{H}$ NMR $(400 \mathrm{MHz}$, Chloroform- $d$ ) $87.25(\mathrm{~d}, J=6.5 \mathrm{~Hz}, 2 \mathrm{H}), 6.83(\mathrm{~d}, J=8.9 \mathrm{~Hz}, 2 \mathrm{H}), 3.92(\mathrm{t}, J=6.5 \mathrm{~Hz}, 2 \mathrm{H}), 2.43(\mathrm{~s}, 3 \mathrm{H})$, $2.21(\mathrm{t}, J=7.5 \mathrm{~Hz}, 2 \mathrm{H}), 1.78-1.75(\mathrm{~m}, 2 \mathrm{H}), 1.64-1.56(\mathrm{~m}, 2 \mathrm{H}), 1.43(\mathrm{~s}, 9 \mathrm{H}), 1.40-1.33(\mathrm{~m}, 4 \mathrm{H}) .{ }^{13} \mathbf{C ~ N M R}(101 \mathrm{MHz}$, $\left.\mathrm{CDCl}_{3}\right) \delta 173.2,157.7,130.2,128.5,115.2,80.0,68.0,35.5,29.1,28.8,28.1,25.8,25.0,18.2$. HRMS (EI): calcd for [M] ${ }^{+}$ $\mathrm{C}_{18} \mathrm{H}_{28} \mathrm{O}_{3} \mathrm{~S} 324.1754$, found: 324.1759 .<smiles>COC(O)CCCCCCOc1cccc(C)c1</smiles>

tert-butyl 7-(3-cyanophenoxy)heptanoate (2o)

The title compound was prepared from 3-((6-iodohexyl)oxy)benzonitrile $(65.8 \mathrm{mg}, 0.2 \mathrm{mmol})$, according to General Procedure A. The crude residue was purified by fla sh chromatography (pentane/EA=100:1, $\mathrm{Rf}=0.30$ ) to give the product as a colorless oil $(48.5 \mathrm{mg}, 80 \%)$.

${ }^{1} \mathbf{H}$ NMR $(300 \mathrm{MHz}$, Chloroform- $d$ ) $87.33-7.38(\mathrm{~m}, 1 \mathrm{H}), 7.22(\mathrm{dt}, J=7.6,1.2 \mathrm{~Hz}, 1 \mathrm{H}), 7.14-7.07(\mathrm{~m}, 2 \mathrm{H}), 3.96(\mathrm{t}, J=6.4$ $\mathrm{Hz}, 2 \mathrm{H}), 2.23(\mathrm{t}, J=7.4 \mathrm{~Hz}, 2 \mathrm{H}), 1.84-1.75(\mathrm{~m}, 2 \mathrm{H}), 1.65-1.56(\mathrm{~m}, 2 \mathrm{H}), 1.44(\mathrm{~s}, 9 \mathrm{H}), 1.42-1.27(\mathrm{~m}, 4 \mathrm{H}) .{ }^{13} \mathbf{C N M R}(75$ $\left.\mathrm{MHz}, \mathrm{CDCl}_{3}\right) \delta 173.1,159.2,130.3,124.3,119.8,118.8,117.3,113.1,80.0,68.2,35.4,28.8,28.7,28.1,25.7$, 24.9. HRMS (EI): calcd for $[\mathrm{M}]^{+} \mathrm{C}_{18} \mathrm{H}_{25} \mathrm{O}_{3} \mathrm{~N} 303.1829$, found: 303.1837 . 
$\overbrace{0}$

tert-butyl 7-(naphthalen-2-yloxy)heptanoate $(\mathbf{2 p})$

The title compound was prepared from 3-((6-iodohexyl)oxy)benzonitrile $(70.8 \mathrm{mg}, 0.2 \mathrm{mmol})$, according to General

Procedure A. The crude residue was purified by fla sh chromatography (pentane/EA=100: $1, \mathrm{Rf}=0.30$ ) to give the product as a colorless oil (56.4 $\mathrm{mg}, 86 \%)$.

${ }^{1} \mathbf{H}$ NMR (400 MHz, Chloroform- $d$ ) $\delta 7.82-7.69(\mathrm{~m}, 3 \mathrm{H}), 7.45-7.41(\mathrm{~m}, 1 \mathrm{H}), 7.35-7.31(\mathrm{~m}, 1 \mathrm{H}), 7.18-7.11(\mathrm{~m}, 2 \mathrm{H})$, $4.07(\mathrm{t}, J=6.5 \mathrm{~Hz}, 2 \mathrm{H}), 2.25(\mathrm{t}, J=7.5 \mathrm{~Hz}, 2 \mathrm{H}), 1.90-1.83(\mathrm{~m}, 2 \mathrm{H}), 1.69-1.62(\mathrm{~m}, 2 \mathrm{H}), 1.58-1.52(\mathrm{~m}, 2 \mathrm{H}), 1.46(\mathrm{~s}, 9 \mathrm{H})$, $1.44-1.39(\mathrm{~m}, 2 \mathrm{H}) .{ }^{13} \mathbf{C}$ NMR $\left(101 \mathrm{MHz} \mathrm{CDCl}_{3}\right) \delta 173.2,157.0,134.6,129.3,128.8,127.6,126.7,126.2,123.4,119.0$, 106.5, 80.0, 67.8, 35.5, 29.1, 28.8, 28.1,25.8, 25.0. HRMS (EI): calcd for [M] ${ }^{+} \mathrm{C}_{21} \mathrm{H}_{28} \mathrm{O}_{3} 328.2033$, found: 328.2040 .

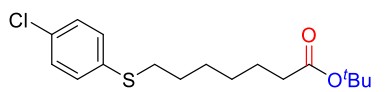

tert-butyl 7-((4-chlorophenyl)thio)heptanoate $(\mathbf{2 q})$

The title compound was prepared from (4-chlorophenyl)(6-iodohexyl)sulfane (70.8 mg, $0.2 \mathrm{mmol}$ ), according to General Procedure A. The crude residue was purified by flash chromatography (pentane/EA $=100: 1, \mathrm{Rf}=0.30$ ) to give the product as a colorless oil (58.4 mg, $89 \%)$.

${ }^{1}$ H NMR $(400 \mathrm{MHz}$, Chloroform- $d) \delta 7.24(\mathrm{~d}, J=0.7 \mathrm{~Hz}, 4 \mathrm{H}), 2.92-2.83(\mathrm{~m}, 2 \mathrm{H}), 2.20(\mathrm{t}, J=7.4 \mathrm{~Hz}, 2 \mathrm{H}), 1.67-1.56(\mathrm{~m}$, 4H), $1.44(\mathrm{~s}, 9 \mathrm{H}), 1.35-1.28(\mathrm{~m}, 4 \mathrm{H}) .{ }^{13} \mathbf{C ~ N M R}\left(101 \mathrm{MHz}, \mathrm{CDCl}_{3}\right) \delta 173.1,135.4,131.7,130.3,129.0,80.0,35.5,33.8$, 31.5, 30.1, 28.8, 28.1, 24.9. HRMS (EI): calcd for $[\mathrm{M}]^{+} \mathrm{C}_{17} \mathrm{H}_{25} \mathrm{O}_{2} \mathrm{ClS} 328.1258$, found: 328.1256 .

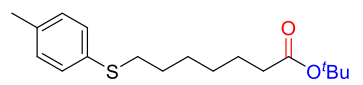

tert-butyl 7-(p-tolylthio)heptanoate (2r)

The title compound was prepared from (6-iodohexyl)( $p$-tolyl)sulfane (66.8 mg, $0.2 \mathrm{mmol}$ ), a ccording to General Procedure A. The crude residue wa s purified by flash chromatography (pentane/EA $=100: 1, \mathrm{Rf}=0.30$ ) to give the product as a colorless oil $(44.4 \mathrm{mg}, 72 \%)$.

${ }^{1} \mathbf{H}$ NMR $(400 \mathrm{MHz}$, Chloroform- $d) \delta 7.24(\mathrm{~d}, J=8.1 \mathrm{~Hz}, 2 \mathrm{H}), 7.09(\mathrm{~d}, J=8.5 \mathrm{~Hz}, 2 \mathrm{H}), 2.90-2.82(\mathrm{~m}, 2 \mathrm{H}), 2.31(\mathrm{~s}, 3 \mathrm{H})$, $2.19(\mathrm{t}, J=7.5 \mathrm{~Hz}, 2 \mathrm{H}), 1.65-1.53(\mathrm{~m}, 6 \mathrm{H}), 1.44(\mathrm{~s}, 9 \mathrm{H}), 1.33-1.28(\mathrm{~m}, 2 \mathrm{H}) .{ }^{13} \mathbf{C ~ N M R}\left(101 \mathrm{MHz}, \mathrm{CDCl}_{3}\right) \delta 173.2,135.9$, 133.0, 129.9, 129.6, 80.0, 35.5, 34.3, 29.1 , 28.6, 28.4, 28.1, 25.0, 21.0. HRMS (EI): calcd for [M] ${ }^{+} \mathrm{C}_{18} \mathrm{H}_{28} \mathrm{O}_{2} \mathrm{~S} 308.1805$, found: 308.1800 .<smiles>CCCCCCCCC(=O)OC(=O)CCCCCn1cccc1</smiles>

tert-butyl 7-(1H-pyrrol-1-yl)heptanoate (2s)

The title compound was prepared from 1-(6-iodohexyl)-1 $H$-pyrrole (55.4 mg, $0.2 \mathrm{mmol})$, a ccording to General Procedure A. The crude residue was purified by flash chromatography (pentane/EA $=100: 1, \mathrm{Rf}=0.30$ ) to give the product as a colorless oil (48.7 mg, 97\%).

${ }^{1}$ H NMR $(300 \mathrm{MHz}$, Chloroform- $d) \delta 6.64(\mathrm{t}, J=2.1 \mathrm{~Hz}, 2 \mathrm{H}), 6.13(\mathrm{t}, J=2.1 \mathrm{~Hz}, 2 \mathrm{H}), 3.86(\mathrm{t}, J=7.1 \mathrm{~Hz}, 2 \mathrm{H}), 2.20(\mathrm{t}, J=$ $7.4 \mathrm{~Hz}, 2 \mathrm{H}), 1.77(\mathrm{p}, J=7.4 \mathrm{~Hz}, 2 \mathrm{H}), 1.62-1.53(\mathrm{~m}, 2 \mathrm{H}), 1.44(\mathrm{~s}, 9 \mathrm{H}), 1.30-1.34(\mathrm{~m}, 4 \mathrm{H}) .{ }^{13} \mathbf{C ~ N M R}\left(75 \mathrm{MHz} \mathrm{CDCl}_{3}\right) \delta$ 173.1 , 120.4, 107.8, 80.0, 49.5, 35.4, 31.4, 28.6, 28.1, 26.4, 24.9. HRMS (EI): calcd for $[\mathrm{M}]^{+} \mathrm{C}_{15} \mathrm{H}_{25} \mathrm{O}_{2} \mathrm{~N} 251.1880$, found: 251.1880 .

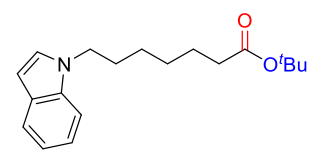

tert-butyl 7-(1H-indol-1-yl)heptanoate (2t)

The title compound wa s prepared from 1-(6-iodohexyl)-1 $H$-indole (65.4 mg, $0.2 \mathrm{mmol})$, a ccording to General Procedure A. The crude residue was purified by flash chromatography (pentane/EA $=100: 1, \mathrm{Rf}=0.30$ ) to give the product as a colorless oil $(57.8 \mathrm{mg}, 96 \%)$.

${ }^{1}$ H NMR $(300 \mathrm{MHz}$, Chloroform- $d) \delta 7.63(\mathrm{ddd}, J=7.8,1.2,0.7 \mathrm{~Hz}, 1 \mathrm{H}), 7.34(\mathrm{dd}, J=8.2,0.9 \mathrm{~Hz}, 1 \mathrm{H}), 7.23-7.17(\mathrm{~m}, 1 \mathrm{H})$, $7.12-7.07(\mathrm{~m}, 2 \mathrm{H}), 6.48(\mathrm{dd}, J=3.1,0.8 \mathrm{~Hz}, 1 \mathrm{H}), 4.11(\mathrm{t}, J=7.1 \mathrm{~Hz}, 2 \mathrm{H}), 2.18(\mathrm{t}, J=7.4 \mathrm{~Hz}, 2 \mathrm{H}), 1.90-1.81(\mathrm{~m}, 2 \mathrm{H}), 1.59$ $-1.51(\mathrm{~m}, 2 \mathrm{H}), 1.43(\mathrm{~s}, 9 \mathrm{H}), 1.37-1.32(\mathrm{~m}, 4 \mathrm{H}) .{ }^{13} \mathbf{C ~ N M R}\left(75 \mathrm{MHz}, \mathrm{CDCl}_{3}\right) \delta 173.1,135.9,128.5,127.7,121.3,120.9$, 119.1, 109.3, 100.9, 80.0, 46.3, 35.4, 30.1, 28.7, 27.5, 26.7, 24.9. HRMS (EI): calcd for [M] ${ }^{+} \mathrm{C}_{19} \mathrm{H}_{27} \mathrm{O}_{2} \mathrm{~N} 301.2036$, found: 301.2036.

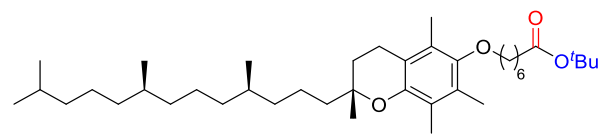

tert-butyl2-(((R)-2,5,7,8-tetramethyl-2-((4R,8R)-4,8,12-trimethyltridecyl)chroman-6-yl)oxy)acetate (2u) 
The title compound was prepared from (R)-6-((6-iodohexyl)oxy)-2,5,7,8-tetramethyl-2-((4R,8R)-4,8,12trimethyltridecyl)chromane (128.1 mg, $0.2 \mathrm{mmol}$ ), according to General Procedure A. The crude residue was purified by flash chromatography (pentane/EA $=100: 1, \mathrm{Rf}=0.30)$ to give the product as a white solid $(72.5 \mathrm{mg}, 59 \%)$.

${ }^{1} \mathbf{H}$ NMR (300 MHz, Chloroform- $\left.d\right) \delta 3.63(\mathrm{t}, J=6.6 \mathrm{~Hz}, 2 \mathrm{H}), 2.57(\mathrm{t}, J=6.7 \mathrm{~Hz}, 2 \mathrm{H}), 2.23(\mathrm{t}, J=7.5 \mathrm{~Hz}, 2 \mathrm{H}), 2.16(\mathrm{~s}, 3 \mathrm{H})$, $2.12(\mathrm{~s}, 3 \mathrm{H}), 2.08(\mathrm{~s}, 3 \mathrm{H}), 1.84-1.73(\mathrm{~m}, 4 \mathrm{H}), 1.69-1.48(\mathrm{~m}, 8 \mathrm{H}), 1.45(\mathrm{~s}, 9 \mathrm{H}), 1.40-1.32(\mathrm{~m}, 6 \mathrm{H}), 1.30-1.21(\mathrm{~m}, 12 \mathrm{H})$, $1.14-1.06(\mathrm{~m}, 4 \mathrm{H}), 0.86(\mathrm{t}, J=7.0 \mathrm{~Hz}, 12 \mathrm{H}) .{ }^{13} \mathbf{C} \mathbf{N M R}\left(75 \mathrm{MHz}, \mathrm{CDCl}_{3}\right) \delta 173.2,148.3,147.6,127.8,125.8,122.7,117.4$, 79.9, 74.7, 72.9, 40.1, 39.4, 37.4, 37.3, 35.6, 32.8, 32.7, 31.5, 31.3, 30.2, 29.1, 28.1, 28.0, 26.0, 25.1, 24.8, 24.4, 23.9, 22.7, 22.6, 21.0, 20.6, 19.7, 19.7, 12.7, 11.9, 11.8. HRMS (EI): calcd for [M] ${ }^{+} \mathrm{C}_{35} \mathrm{H}_{60} \mathrm{O}_{4}$ 544.4492, found:544.4495.

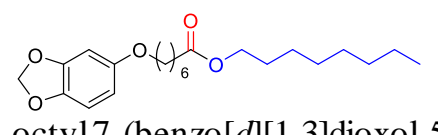

octyl7-(benzo[d][1,3]dioxol-5-yloxy)heptanoate (4a)

The title compound was prepared from 5-((6-iodohexyl)oxy)benzo[d][1,3]dioxole (69.6 mg, $0.2 \mathrm{mmol})$ a nd octan-1-ol(130.1 $\mathrm{mg}, 5$ equiv.), according to General Procedure B. The crude residue was purified by flash chromatography (pentane/EA = $50: 1, \mathrm{Rf}=0.30)$ to give the product as a colorless oil $(41.6 \mathrm{mg}, 55 \%)$.

${ }^{1}$ H NMR (400 MHz, Chloroform- $\left.d\right) \delta 6.69(\mathrm{~d}, J=8.5 \mathrm{~Hz}, 1 \mathrm{H}), 6.48(\mathrm{~d}, J=2.5 \mathrm{~Hz}, 1 \mathrm{H}), 6.30(\mathrm{dd}, J=8.5,2.5 \mathrm{~Hz}, 1 \mathrm{H}), 5.90$ $(\mathrm{s}, 2 \mathrm{H}), 4.06(\mathrm{t}, J=6.8 \mathrm{~Hz}, 2 \mathrm{H}), 3.87(\mathrm{t}, J=6.5 \mathrm{~Hz}, 2 \mathrm{H}), 2.31(\mathrm{t}, J=7.5 \mathrm{~Hz}, 2 \mathrm{H}), 1.78-1.71(\mathrm{~m}, 2 \mathrm{H}), 1.69-1.58(\mathrm{~m}, 4 \mathrm{H})$, $1.55-1.26(\mathrm{~m}, 14 \mathrm{H}), 0.88(\mathrm{t}, J=7.0 \mathrm{~Hz}, 3 \mathrm{H}) .{ }^{13} \mathbf{C N M R}\left(101 \mathrm{MHz}, \mathrm{CDCl}_{3}\right) \delta 173.9,154.6,148.2,141.4,107.9,105.6,101.0$, 98.0, 68.7, 64.5, 34.3, 31.8, 29.2, 29.2, 29.1, 28.9, 28.6, 25.9, 25.7, 24.9, 22.6, 14.1. HRMS (EI): calcd for [M] ${ }^{+} \mathrm{C}_{22} \mathrm{H}_{34} \mathrm{O}_{5}$ 378.2401, found: 378.2398 .

$$
\mathrm{O}^{\circ} \mathrm{O}_{6} \mathrm{~N}_{\mathrm{O}}^{\mathrm{H}} \mathrm{\sim}_{\mathrm{Ph}}
$$

3-phenylpropyl 7-(benzo[d][1,3]dioxol-5-yloxy)heptanoate (4b)

The title compound was prepared from 5-((6-iodohexyl)oxy)benzo[ $d][1,3]$ dioxole $(69.6 \mathrm{mg}, 0.2 \mathrm{mmol})$ and 3-phenylpropan1-ol (136.1 mg, 5 equiv.), according to General Procedure B. The crude residue was purified by fla sh chromatography (pentane/EA $=50: 1, \mathrm{Rf}=0.30)$ to give the product a s a colorless oil $(39.9 \mathrm{mg}, 52 \%)$.

${ }^{1}$ H NMR (400 MHz, Chloroform-d) $\delta 7.36-7.26(\mathrm{~m}, 2 \mathrm{H}), 7.21-7.17(\mathrm{~m}, 3 \mathrm{H}), 6.69(\mathrm{~d}, J=8.5 \mathrm{~Hz}, 1 \mathrm{H}), 6.48(\mathrm{~d}, J=2.5 \mathrm{~Hz}$, $1 \mathrm{H}), 6.31(\mathrm{dd}, J=8.5,2.5 \mathrm{~Hz}, 1 \mathrm{H}), 5.90(\mathrm{~s}, 2 \mathrm{H}), 4.10(\mathrm{t}, J=6.6 \mathrm{~Hz}, 2 \mathrm{H}), 3.87(\mathrm{t}, J=6.5 \mathrm{~Hz}, 2 \mathrm{H}), 2.69(\mathrm{t}, J=7.7 \mathrm{~Hz}, 2 \mathrm{H})$, $2.32(\mathrm{t}, J=7.5 \mathrm{~Hz}, 2 \mathrm{H}), 2.01-1.92(\mathrm{~m}, 2 \mathrm{H}), 1.79-1.72(\mathrm{~m}, 2 \mathrm{H}), 1.70-1.63(\mathrm{~m}, 2 \mathrm{H}), 1.52-1.44(\mathrm{~m}, 2 \mathrm{H}), 1.42-1.34(\mathrm{~m}$, $2 \mathrm{H}) .{ }^{13} \mathrm{C}$ NMR $\left(101 \mathrm{MHz}, \mathrm{CDCl}_{3}\right) \delta 173.8,154.6,148.2,141.5,141.2,128.5,128.4,126.0,107.9,105.6,101.1,98.1,68.8$, 63.6, 34.3, 32.2, 30.3, 29.1, 28.9, 25.8, 24.9. HRMS (EI): calcd for [M] ${ }^{+} \mathrm{C}_{23} \mathrm{H}_{28} \mathrm{O}_{5}$ 384.1931, found:384.1930.<smiles>CCCOC(=O)Oc1ccc2c(c1)OCO2</smiles>

3-(trimethylsilyl)propyl 7-(benzo[d][1,3]dioxol-5-yloxy)heptanoate (4c)

The title compound was prepared from 5-((6-iodohexyl)oxy)benzo[d][1,3]dioxole $(69.6 \mathrm{mg}, 0.2 \mathrm{mmol})$ and 2(trimethylsilyl)ethan-1-ol(118.1 mg, 5 equiv.), a ccording to General Procedure B. The crude residue was purified by flash chromatography (pentane/EA $=50: 1, \mathrm{Rf}=0.30)$ to give the product $(39.5 \mathrm{mg}, 54 \%)$.

${ }^{1}$ H NMR $(300 \mathrm{MHz}$, Chloroform- $d) \delta 6.69(\mathrm{~d}, J=8.5 \mathrm{~Hz}, 1 \mathrm{H}), 6.48(\mathrm{~d}, J=2.4 \mathrm{~Hz}, 1 \mathrm{H}), 6.30(\mathrm{dd}, J=8.5,2.5 \mathrm{~Hz}, 1 \mathrm{H}), 5.90$ $(\mathrm{s}, 2 \mathrm{H}), 4.21-4.11(\mathrm{~m}, 2 \mathrm{H}), 3.87(\mathrm{t}, J=6.5 \mathrm{~Hz}, 2 \mathrm{H}), 2.29(\mathrm{t}, J=7.5 \mathrm{~Hz}, 2 \mathrm{H}), 1.78-1.61(\mathrm{~m}, 4 \mathrm{H}), 1.48-1.37(\mathrm{~m}, 4 \mathrm{H}), 1.01$ $-0.94(\mathrm{~m}, 2 \mathrm{H}), 0.04(\mathrm{~s}, 9 \mathrm{H}) .{ }^{13} \mathbf{C}$ NMR $\left(75 \mathrm{MHz} \mathrm{CDCl}_{3}\right) \delta 175.4,156.1,149.7,142.9,109.4,107.1,102.5,99.5,70.3,63.9$, 35.9, 30.6, 30.4, 27.2, 26.4, 18.8,0.0. HRMS (EI): calcd for $[\mathrm{M}]^{+} \mathrm{C}_{19} \mathrm{H}_{30} \mathrm{O}_{5} \mathrm{Si} 366.1857$, found: 366.1855 .

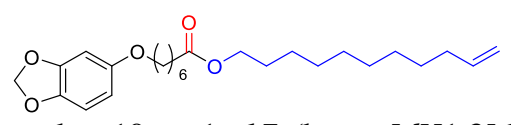

undec-10-en-1-yl 7-(benzo[d][1,3]dioxol-5-yloxy)heptanoate(4d)

The title compound was prepared from 5-((6-iodohexyl)oxy)benzo[d][1,3]dioxole $(69.6 \mathrm{mg}, 0.2 \mathrm{mmol})$ and undec-10-en-1-ol (170.2 mg, 5 equiv.), a ccording to General Procedure B. The crude residue was purified by flash chromatography (pentane/EA $=50: 1, \mathrm{Rf}=0.30)$ to give the $\operatorname{product}(34.3 \mathrm{mg}, 41 \%)$.

${ }^{1}$ H NMR (300 MHz, Chloroform-d) $\delta 6.69(\mathrm{~d}, J=8.5 \mathrm{~Hz}, 1 \mathrm{H}), 6.48(\mathrm{~d}, J=2.5 \mathrm{~Hz}, 1 \mathrm{H}), 6.30(\mathrm{dd}, J=8.5,2.5 \mathrm{~Hz}, 1 \mathrm{H}), 5.90$ $(\mathrm{s}, 2 \mathrm{H}), 5.88-5.72(\mathrm{~m}, 1 \mathrm{H}), 5.03-4.89(\mathrm{~m}, 2 \mathrm{H}), 4.05(\mathrm{t}, J=6.7 \mathrm{~Hz}, 2 \mathrm{H}), 3.87(\mathrm{t}, J=6.5 \mathrm{~Hz}, 2 \mathrm{H}), 2.31(\mathrm{t}, J=7.5 \mathrm{~Hz}, 2 \mathrm{H})$, $2.03(\mathrm{q}, J=6.8 \mathrm{~Hz}, 2 \mathrm{H}), 1.79-1.70(\mathrm{~m}, 2 \mathrm{H}), 1.69-1.58(\mathrm{~m}, 4 \mathrm{H}), 1.47-1.28(\mathrm{~m}, 16 \mathrm{H}) .{ }^{13} \mathbf{C N M R}\left(75 \mathrm{MHz}^{2} \mathrm{CDCl}_{3}\right) \delta 173.9$, 154.6, 148.2, 140.0, 139.2, 114.1, 107.9, 105.6, 101.0,98.0, 68.7, 64.4, 34.3, 33.8, 29.4, 29.4, 29.2, 29.1, 29.1, 28.9, 28.9, 28.6, 25.9, 25.7, 24.9. HRMS (EI): calcd for $[\mathrm{M}]^{+} \mathrm{C}_{25} \mathrm{H}_{38} \mathrm{O}_{5} 418.2714$, found: 418.2716. 
ON

2,4-dimethylpentan-3-yl 7-(benzo[d][1,3]dioxol-5-yloxy)heptanoate (4e)

The title compound was prepared from 5-((6-iodohexyl)oxy)benzo[d][1,3]dioxole $(69.6 \mathrm{mg}, 0.2 \mathrm{mmol})$ and 2,4dimethylpentan-3-ol (116.1 mg, 5 equiv.), according to General Procedure B. The crude residue was purified by flash chromatography (pentane/EA $=50: 1, \mathrm{Rf}=0.30)$ to give the product $(34.9 \mathrm{mg}, 48 \%)$.

${ }^{1} \mathrm{H}$ NMR $(400 \mathrm{MHz}$, Chloroform- $d$ ) $\delta 6.69(\mathrm{~d}, J=8.5 \mathrm{~Hz}, 1 \mathrm{H}), 6.48(\mathrm{~d}, J=2.5 \mathrm{~Hz}, 1 \mathrm{H}), 6.30(\mathrm{dd}, J=8.5,2.5 \mathrm{~Hz}, 1 \mathrm{H}), 5.90$ $(\mathrm{s}, 2 \mathrm{H}), 4.94-4.87(\mathrm{~m}, 1 \mathrm{H}), 3.87(\mathrm{t}, J=6.5 \mathrm{~Hz}, 2 \mathrm{H}), 2.30(\mathrm{t}, J=7.5 \mathrm{~Hz}, 2 \mathrm{H}), 1.78-1.71(\mathrm{~m}, 2 \mathrm{H}), 1.65(\mathrm{p}, J=7.5 \mathrm{~Hz}, 2 \mathrm{H})$, $1.54-1.43(\mathrm{~m}, 6 \mathrm{H}), 1.35-1.27(\mathrm{~m}, 6 \mathrm{H}), 0.90(\mathrm{t}, J=7.3 \mathrm{~Hz}, 6 \mathrm{H}) .{ }^{13} \mathbf{C} \mathbf{N M R}\left(101 \mathrm{MHz}, \mathrm{CDCl}_{3}\right) \delta 173.6,154.6,148.2,141.4$, $107.9,105.6,101.0,98.0,73.6,68.7,36.3,34.6,29.1,28.9,25.7,25.0,18.6,14.0$. HRMS (EI): calcd for $[\mathrm{M}]^{+} \mathrm{C}_{21} \mathrm{H}_{32} \mathrm{O}_{5}$ 364.2244, found: 364.2242 .

\section{Mechanistic Studies}

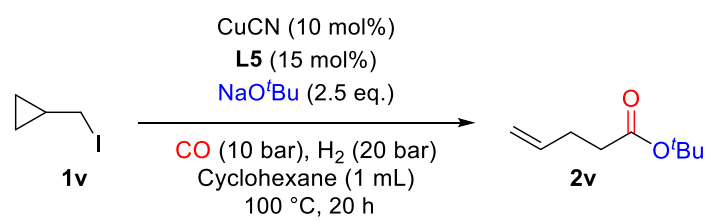

The $2 \mathbf{v}^{[9]}$ was prepared from (iodomethyl)cyclopropane $1 \mathbf{v}(36.4 \mathrm{mg}, 0.2 \mathrm{mmol})$, a ccording to General Procedure A.

${ }^{1} \mathbf{H}$ NMR $(400 \mathrm{MHz}$, Chloroform- $d) \delta 5.75-5.55(\mathrm{~m}, 1 \mathrm{H}), 4.96-4.79(\mathrm{~m}, 2 \mathrm{H}), 2.22-2.08(\mathrm{~m}, 4 \mathrm{H}), 1.29(\mathrm{~s}, 9 \mathrm{H}) .{ }^{13} \mathbf{C N M R}$ $\left(101 \mathrm{MHz}, \mathrm{CDCl}_{3}\right) \delta 172.4,137.0,115.2,86.1,34.7,28.4,28.1$.

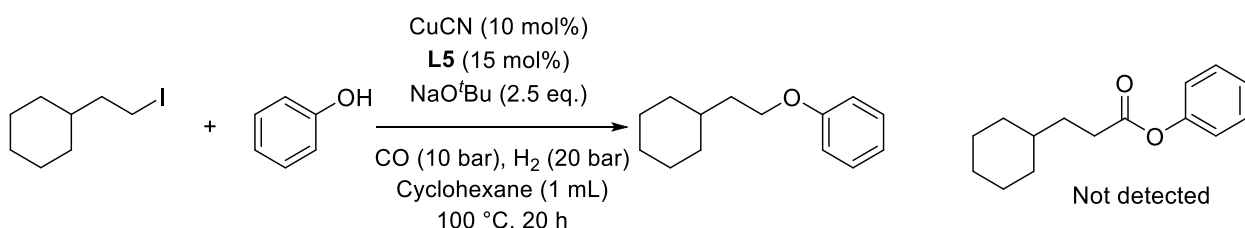

\section{References}

[1] Y. Gao, C. Yang, S. Bai, X. Liu, Q. Wu, J. Wang, C. Jiang, X. Qi, Visible-Light-Induced Nickel-Catalyzed CrossCoupling with Alkylzirconocenes from Unactivated Alkenes. Chem, 2020, 6, 675-688.

[2] Q. Liu, J. Hong, B. Sun, G. Bai, F. Li, G. Liu, Y. Yang, F. Mo, Transition-metal-free borylation of alkyl iodides via a radicalmechanism. Org. Lett. 2019, 21,6597-6602.

[3] X. T. Zhou, R. G. Carter, Synthesis of the C1-C26 Northern Portion of Azaspiracid-1: Kinetic versus Thermodynamic Control of the Formation of the Bis-spiroketal. Angew. Chem. Int. Ed. 2006, 45, 1787-1790.

[4] S. Zhao, N. P. Mankad, Cu-Catalyzed Hydroxymethylation of Unactivated Alkyl Iodides with CO to Provide OneCarbon-Extended Alcohols. Angew. Chem. Int. Ed. 2018, 57, 5867 -5870.

[5] Y. Iwata, Y. Tanaka, S. Kubosaki, T. Morita, Y. Yoshimi, A Strategy for Generating Aryl Ra dicals from Arylborates through Orga nic Photoredox Cataly sis: Photo-Meerwein Type Arylation of Electron-Deficient Alkenes. Chem. Commun., 2018, 54, 1257-1260.

[6] E. Korytiaková, N. O. Thiel, F. Pape, J. F. Teichert, Copper (I)-Catalysed Transfer Hydrogenations with Ammonia Borane. Chem. Commun., 2017, 53, 732-735.

[7] L. Degennaro, D. Maggiulli, C. Carlucci, F. Fanelli, G. Romanazzi, R. Luisi, A direct a nd sustainable synthesis of tertiary butylesters enabled by flow microreactors. Chem. Commun., 2016, 52, 9554-9557.

[8] C. C. Li, X. J. Dai, H. Wang, D. Zhu, J. Gao, C. J. Li, Iron-Catalyzed Nucleophilic Addition Reaction of Organic Carbanion Equivalents via Hydrazones. Org. Lett. 2018, 20,3801-3805.

[9] M.P. DeMartino, K. Chen, P. S. Baran, Intermolecular Enolate Heterocoupling: Scope, Mechanism, and Application. J. Am. Chem. Soc. 2008, 130, 11546-11560. 


\section{NMR Spectra of the Products}

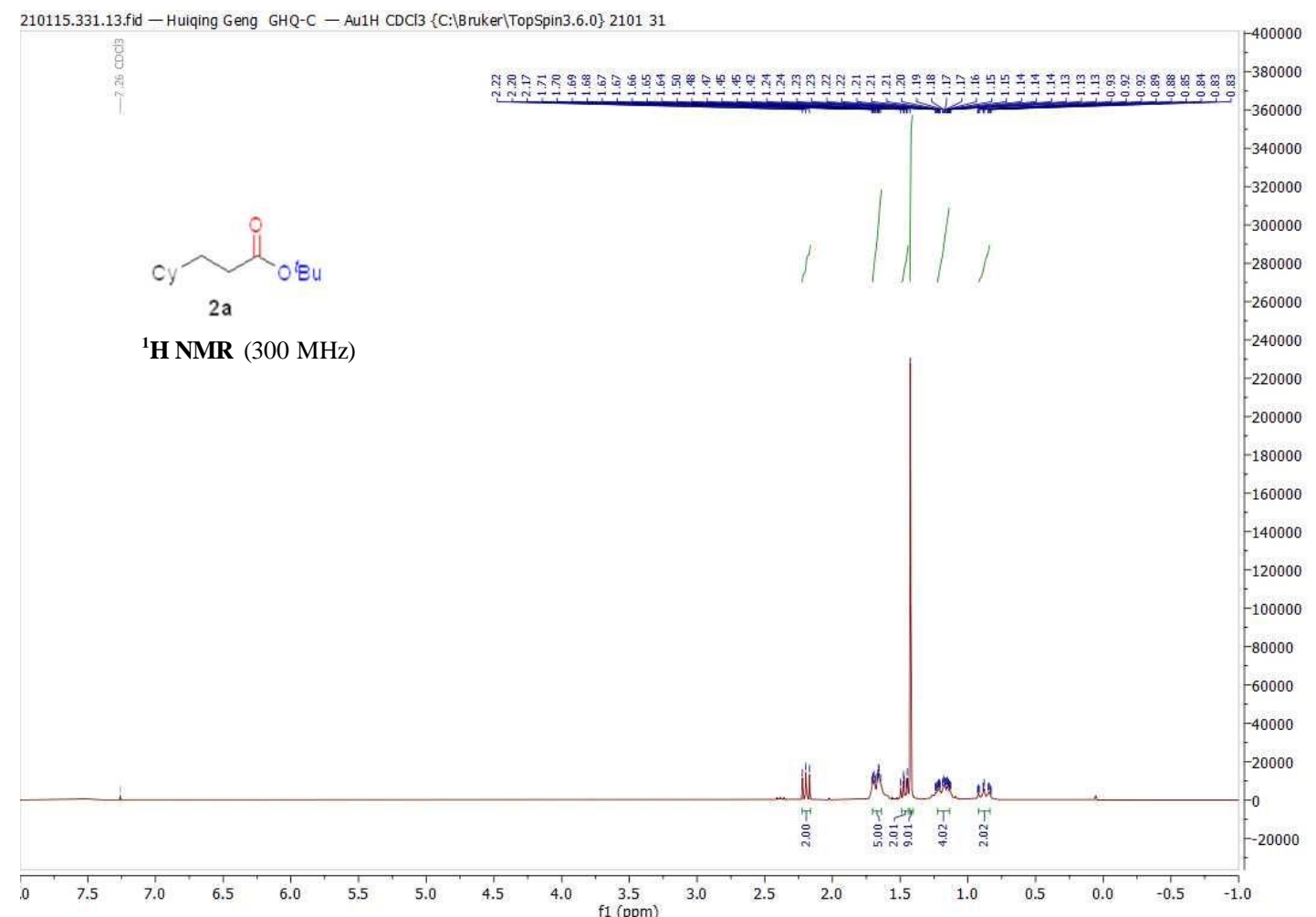

210115.331.12.fid - Huiqing Geng GHQ-C - Au13C CDCl3 \{C:|Bruker|TopSpin3.6.0\} 210131

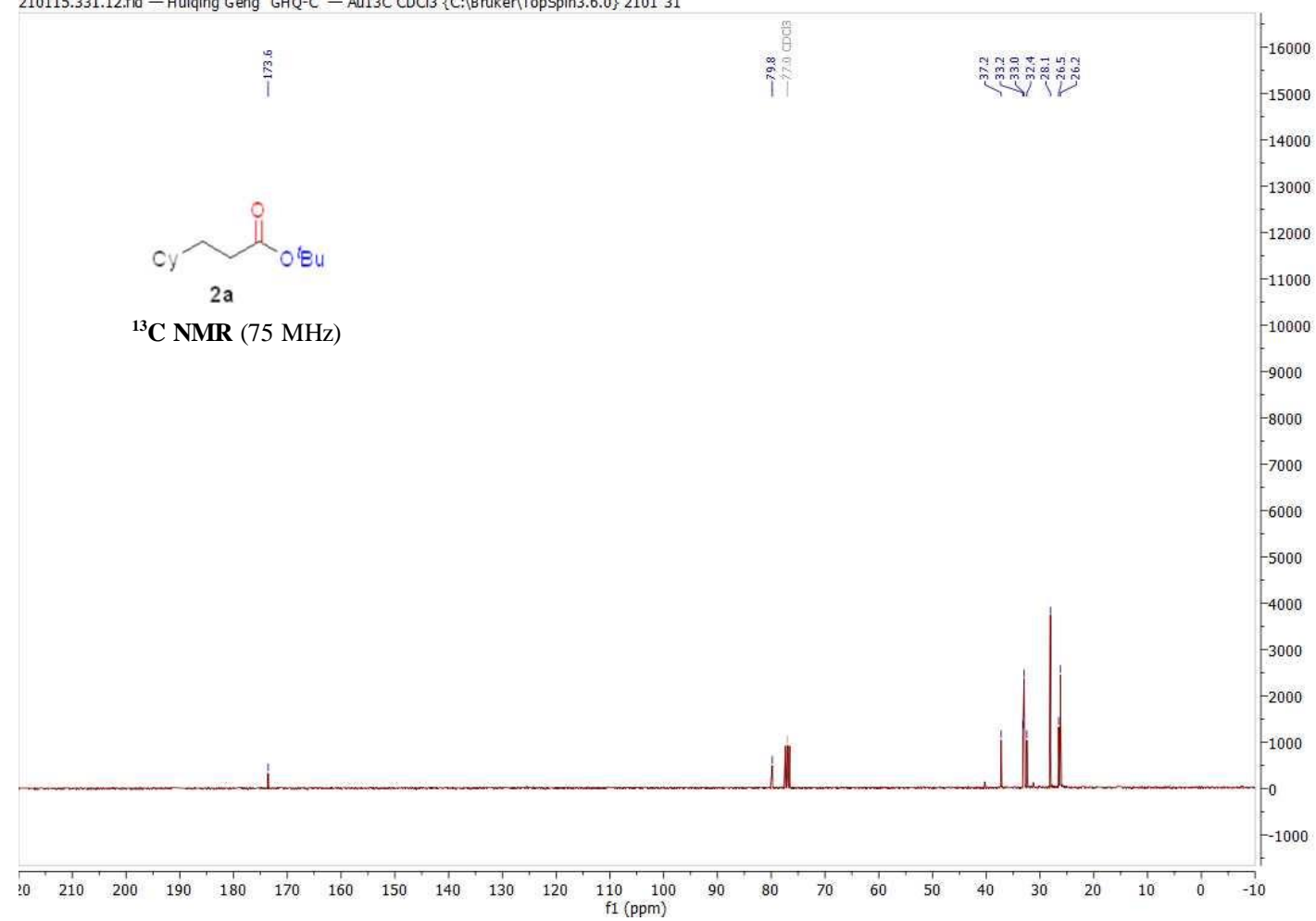



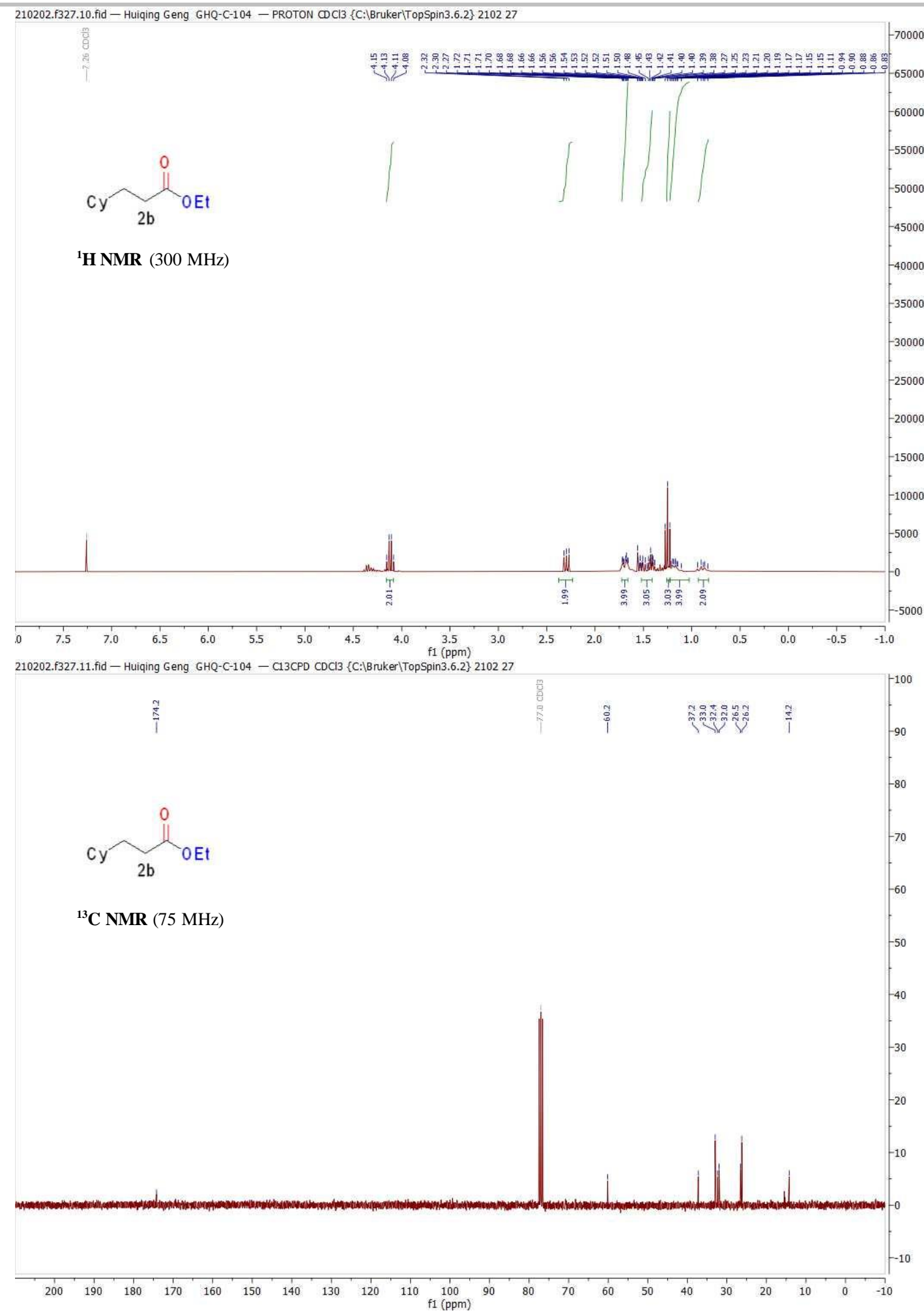


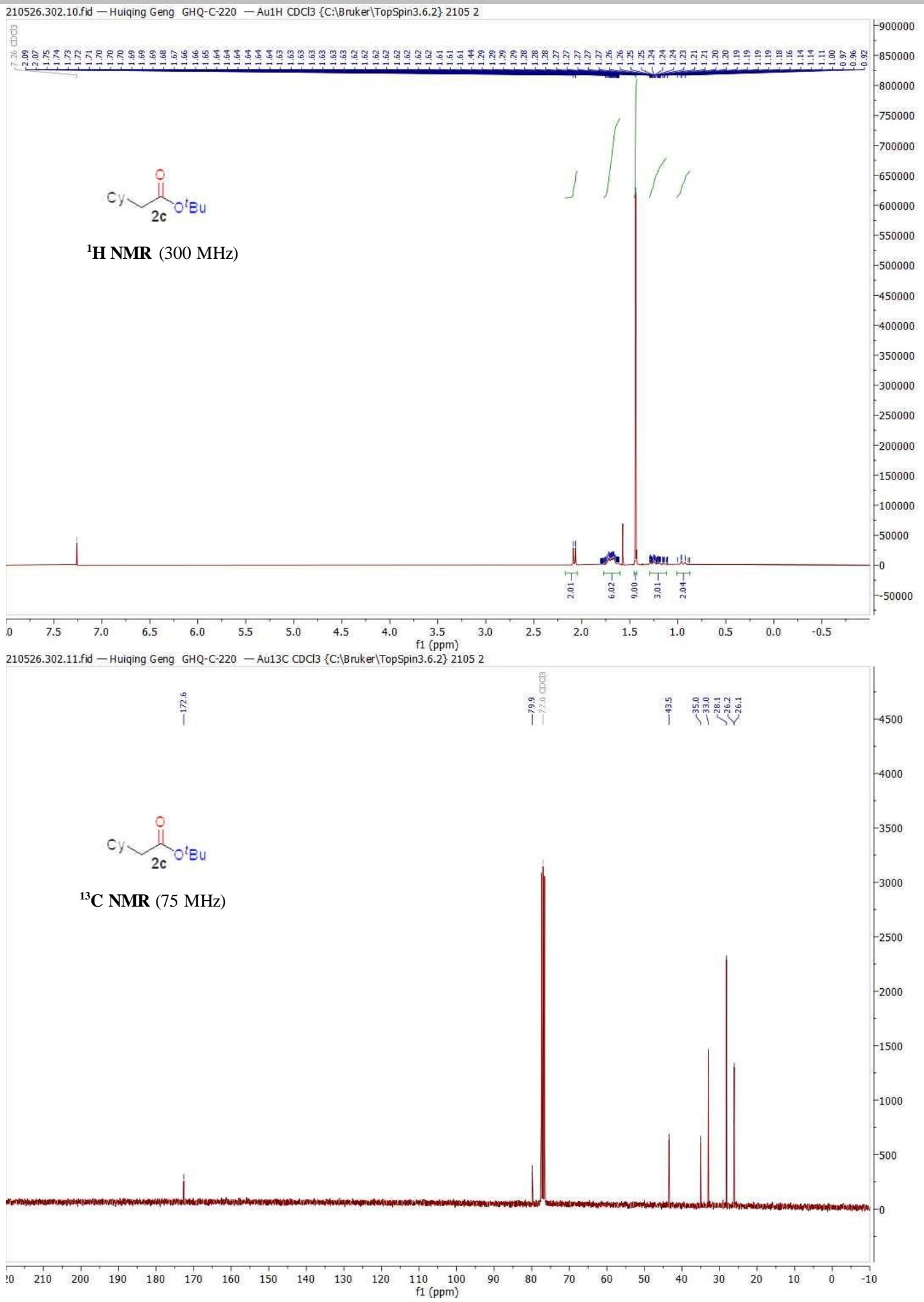



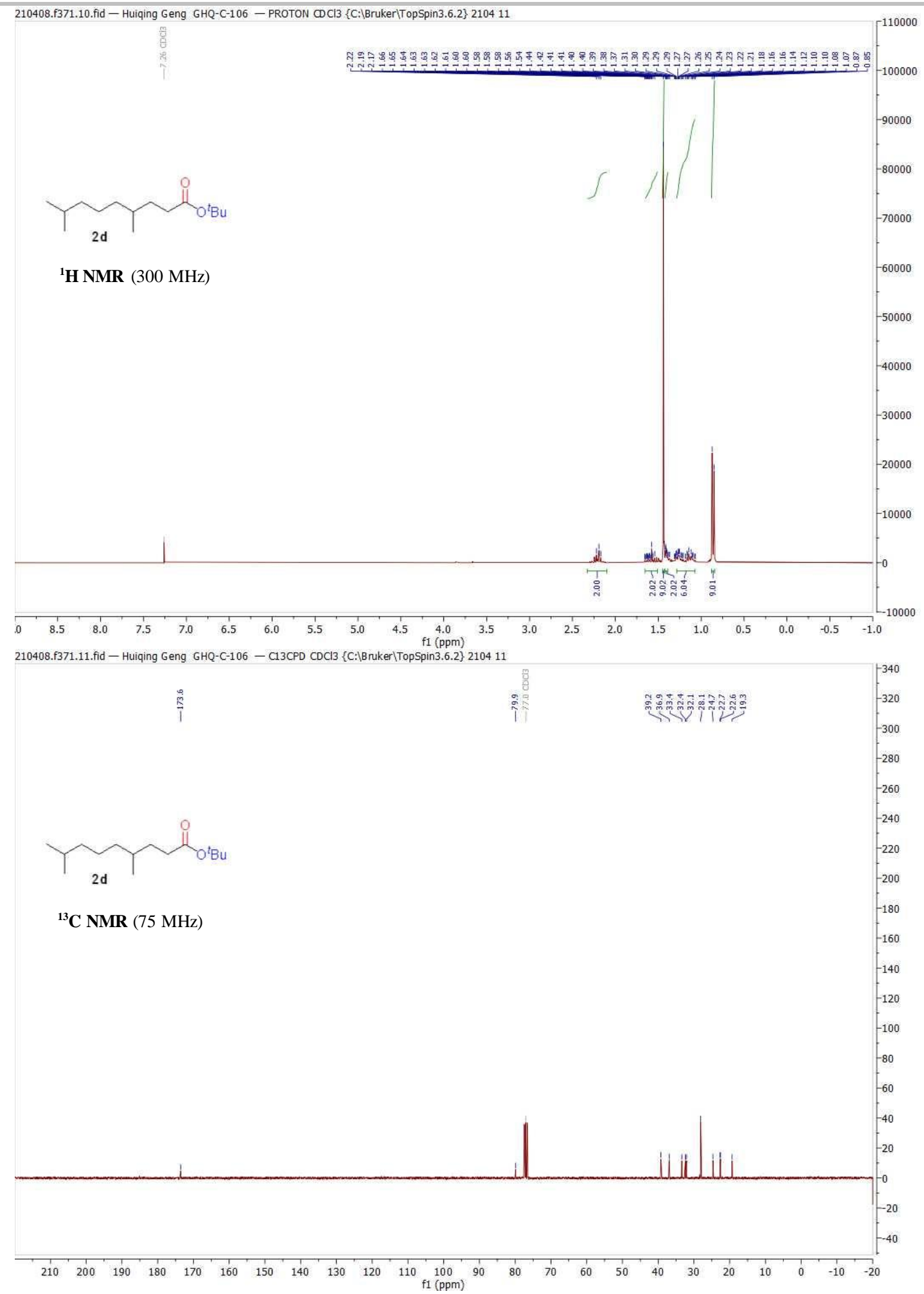
210412.f334.10.fid - Huiqing Geng GHQ-C-93 — PROTON CDCl3 \{C:|Bruker|TopSpin3.6.2\} 210434
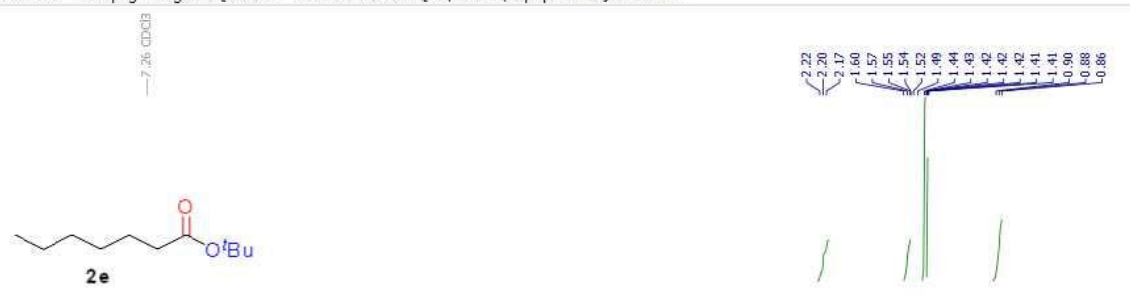

${ }^{1}$ H NMR (300 MHz)
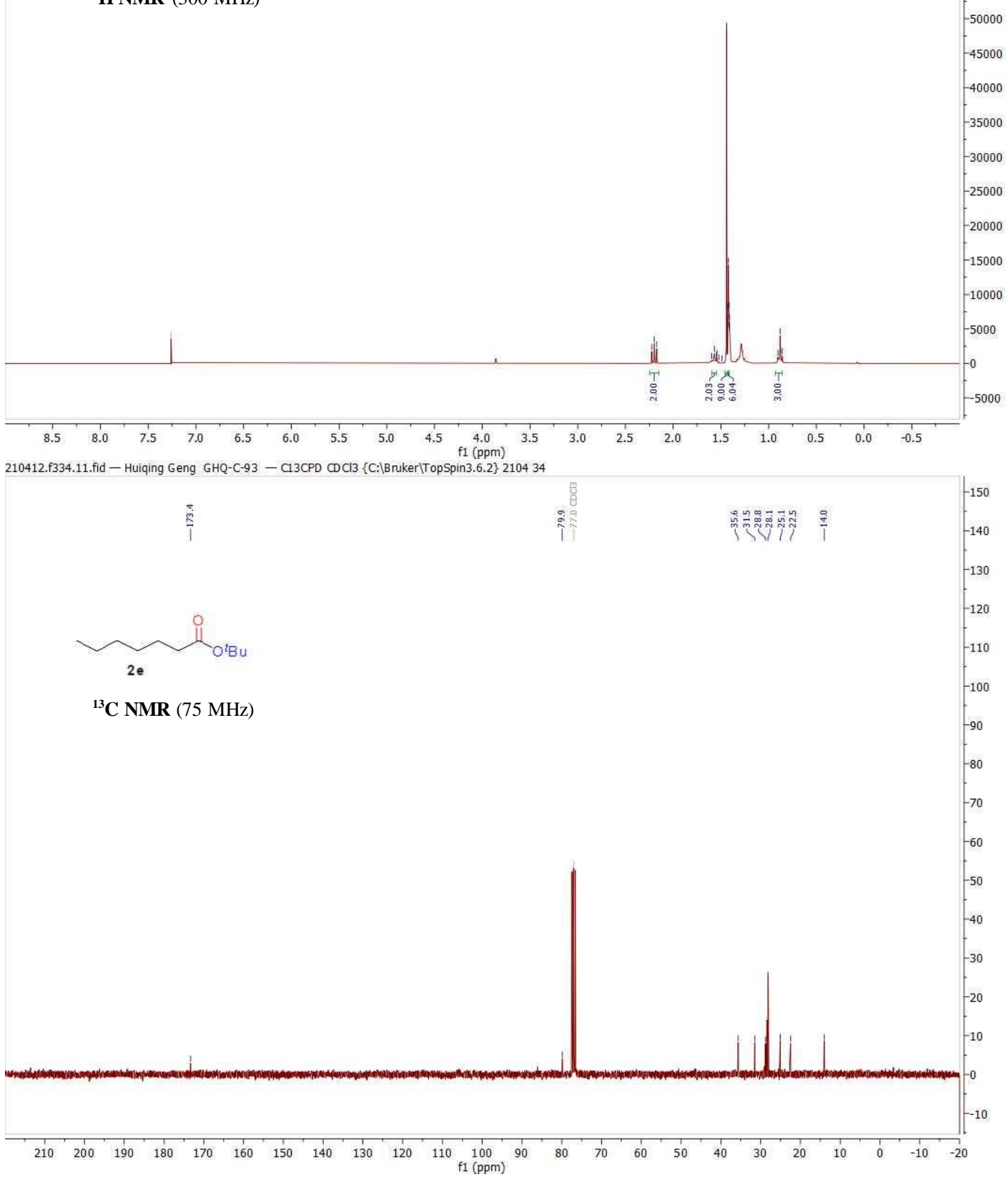
<smiles>CCOC(=O)CCCc1ccccc1</smiles>

$2 f$
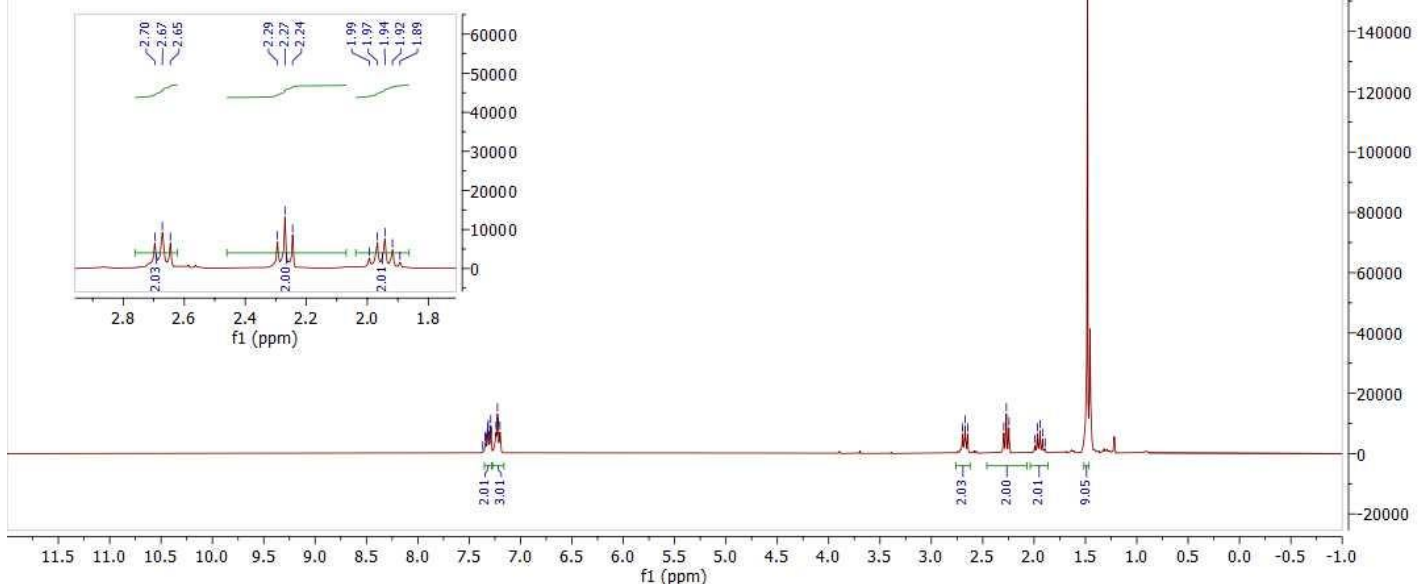

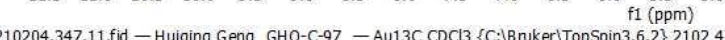

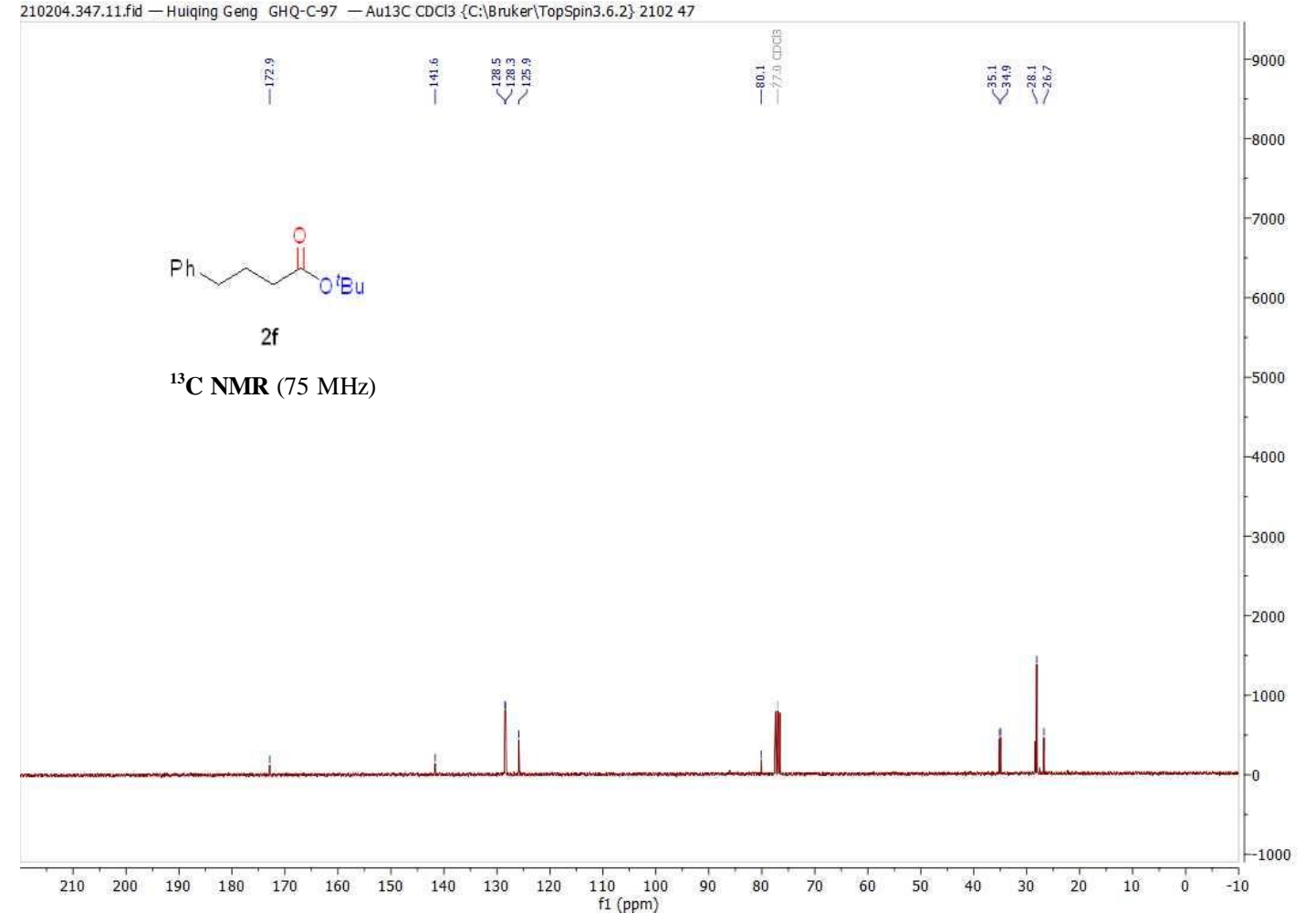


210526.303.10.fid - Huiqing Geng GHQ-C-218 - Au1H CDCl3 \{C:|Bruker|TopSpin3.6.2\} 21053

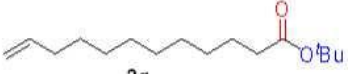

${ }^{1} \mathbf{H}$ NMR $(300 \mathrm{MHz})$

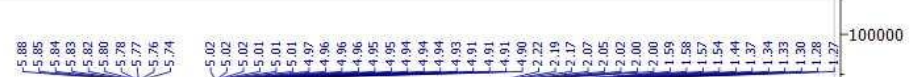

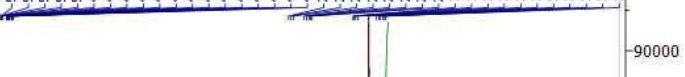

$-90000$

$-80000$

70000

$-60000$

$-50000$

40000

$-30000$

$-2000$

$-10000$

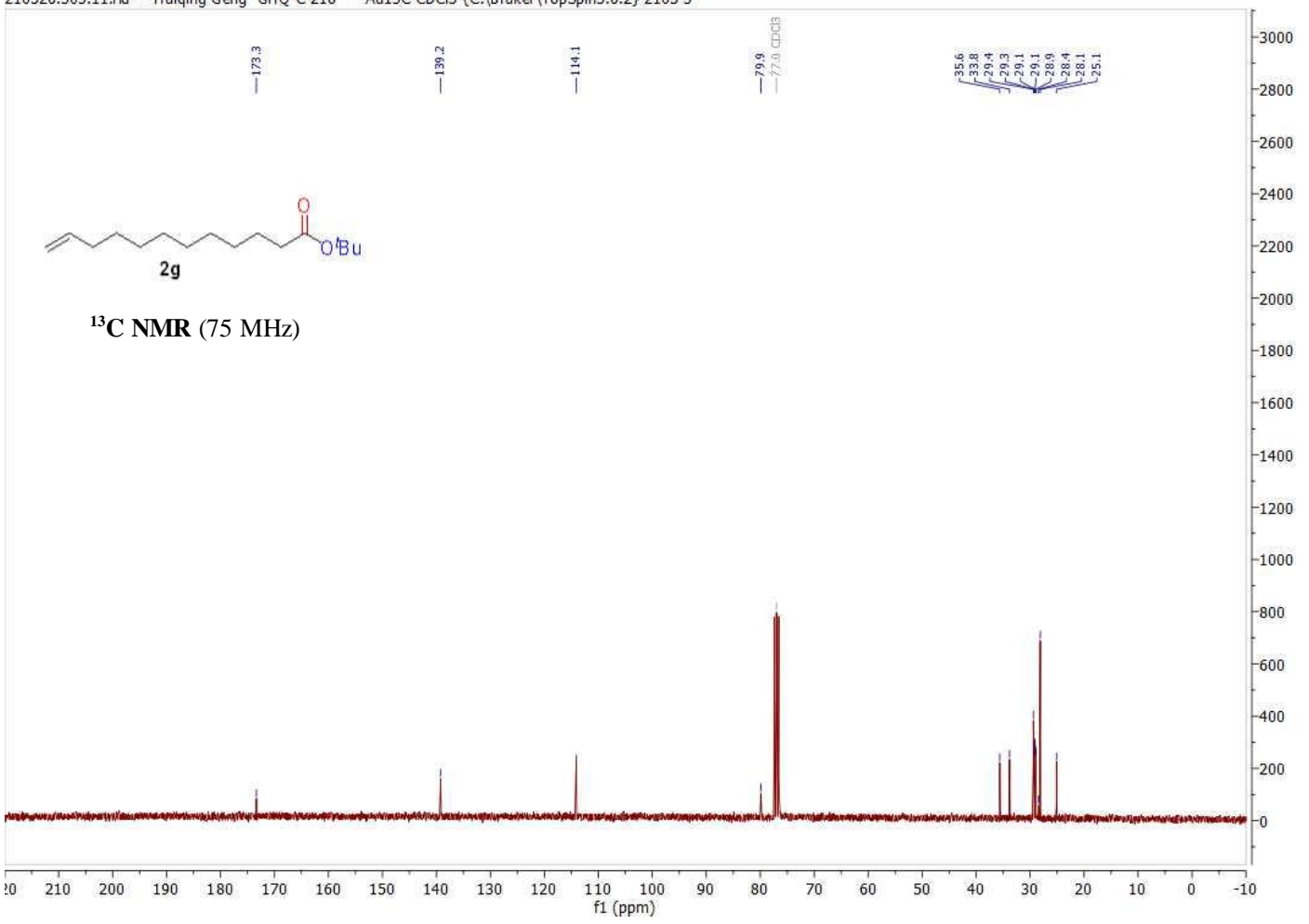

$2 g$
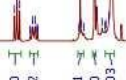

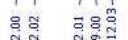

$\begin{array}{lllllllllllllllllllllllllllllllllll}0 & 11.5 & 11.0 & 10.5 & 10.0 & 9.5 & 9.0 & 8.5 & 8.0 & 7.5 & 7.0 & 6.5 & 6.0 & 5.5 & 5.0 & 4.5 & 4.0 & 3.5 & 3.0 & 2.5 & 2.0 & 1.5 & 1.0 & 0.5 & 0.0 & -0.5\end{array}$ 210526.303.11.fid - Huiqing Geng GHQ-C-218 - Au13C CDCl3 \{C:|Bruker|TopSpin3.6.2\} 21053 

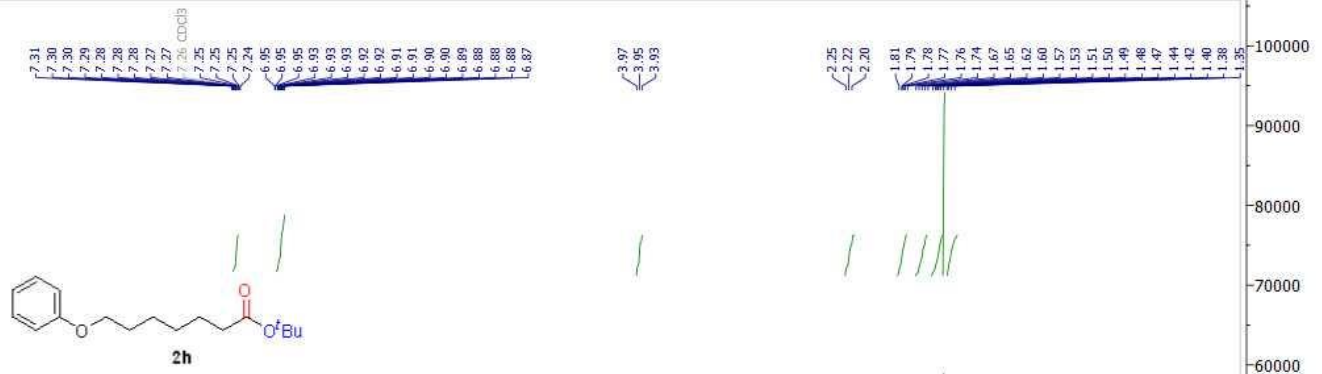

${ }^{1}$ H NMR $(300 \mathrm{MHz})$

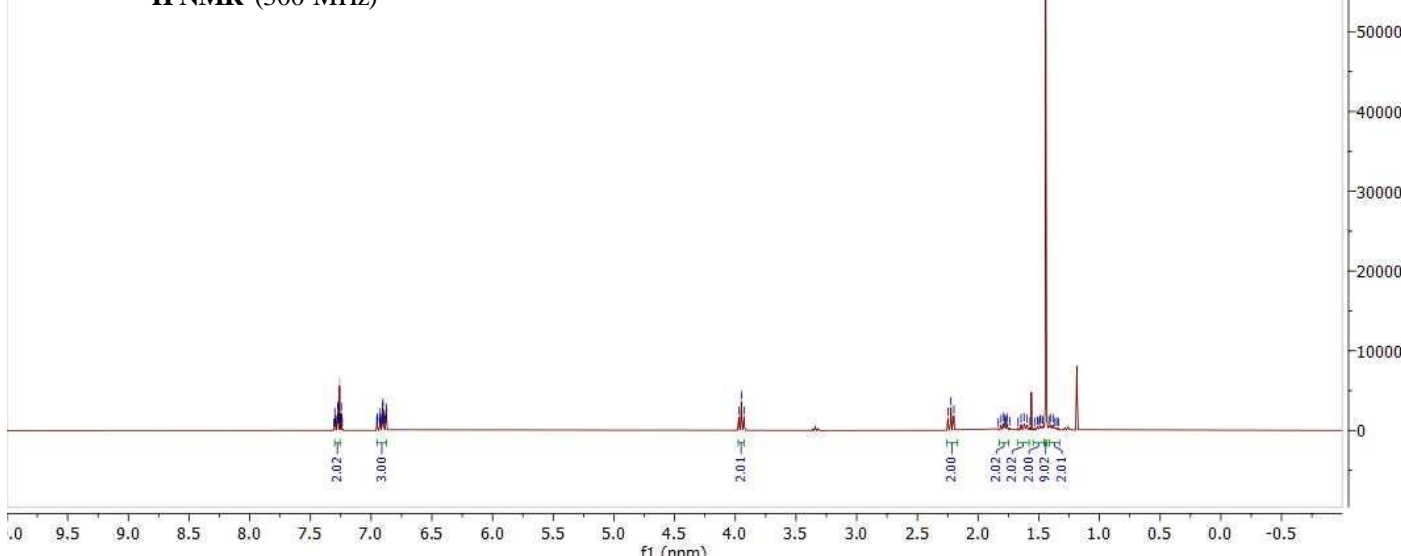
210416.f307.11.fid - Geng/ GHQ-C-138 - C13CPD CDCl3 \{C:|Bruker|TopSpin3.6.2\} 21047

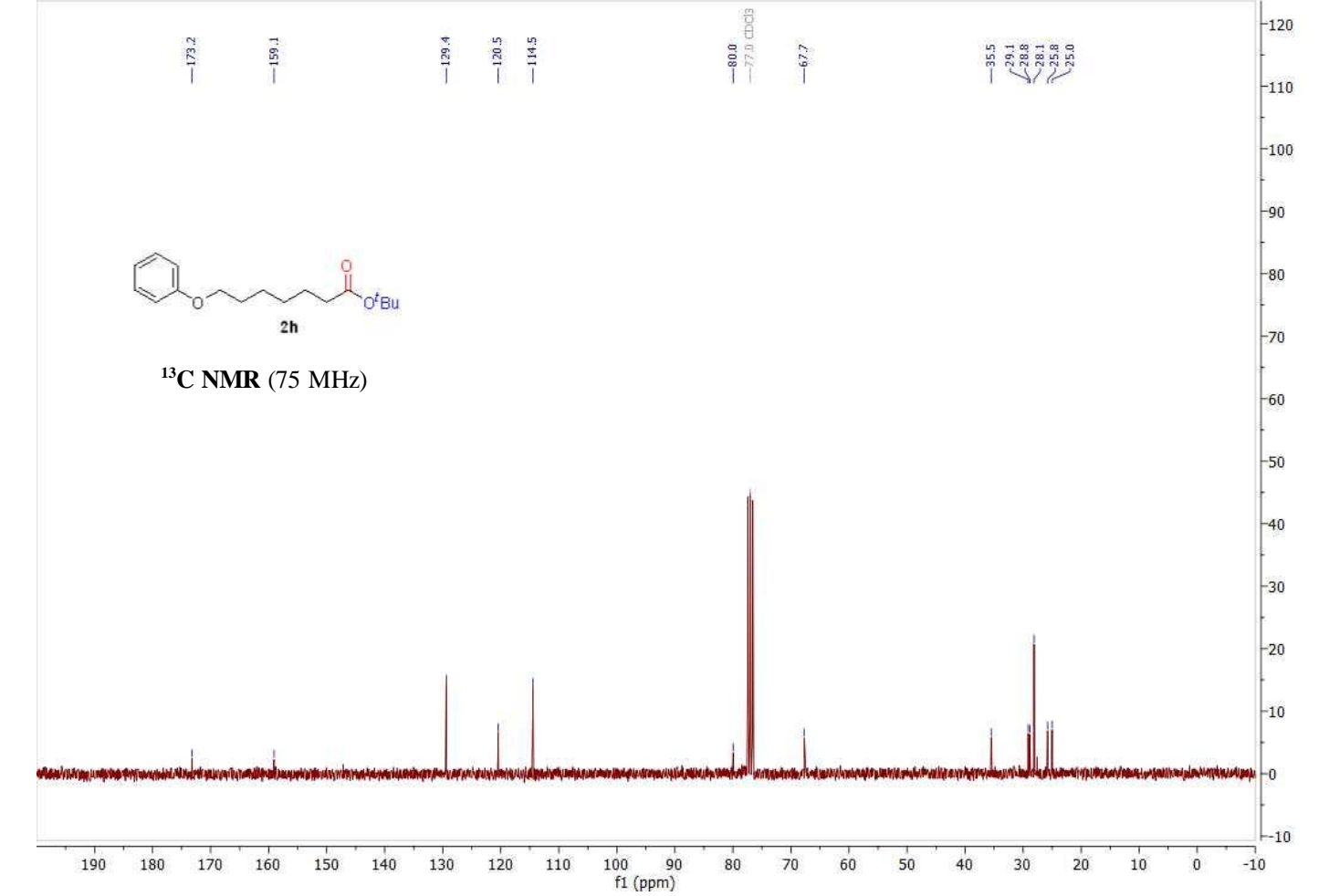


210517.440.10.fid - Huiqing Geng / GHQ-C-206 - Au1H CDCl3 \{C:|Bruker|TopSpin3.5pl6\} 210540

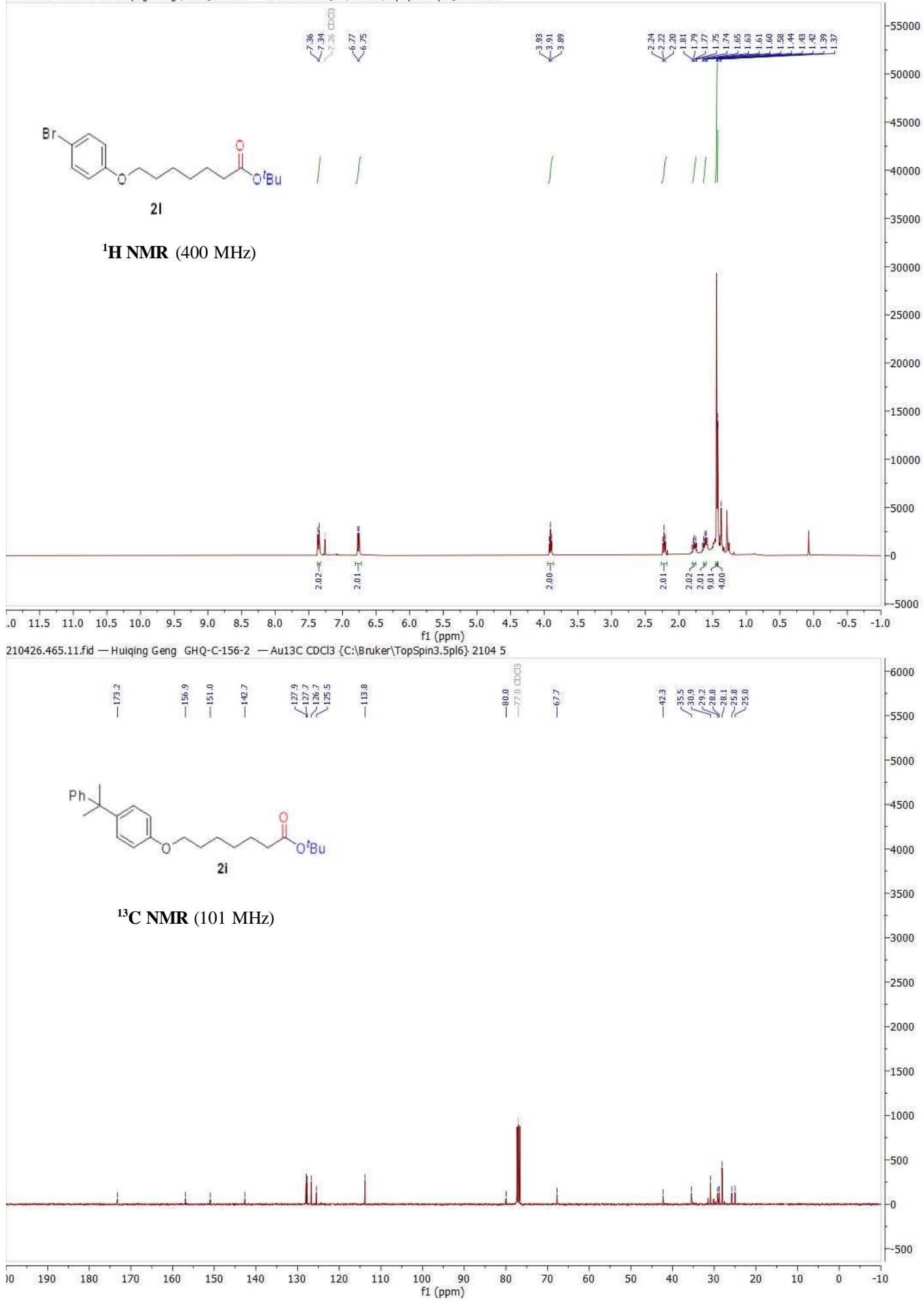




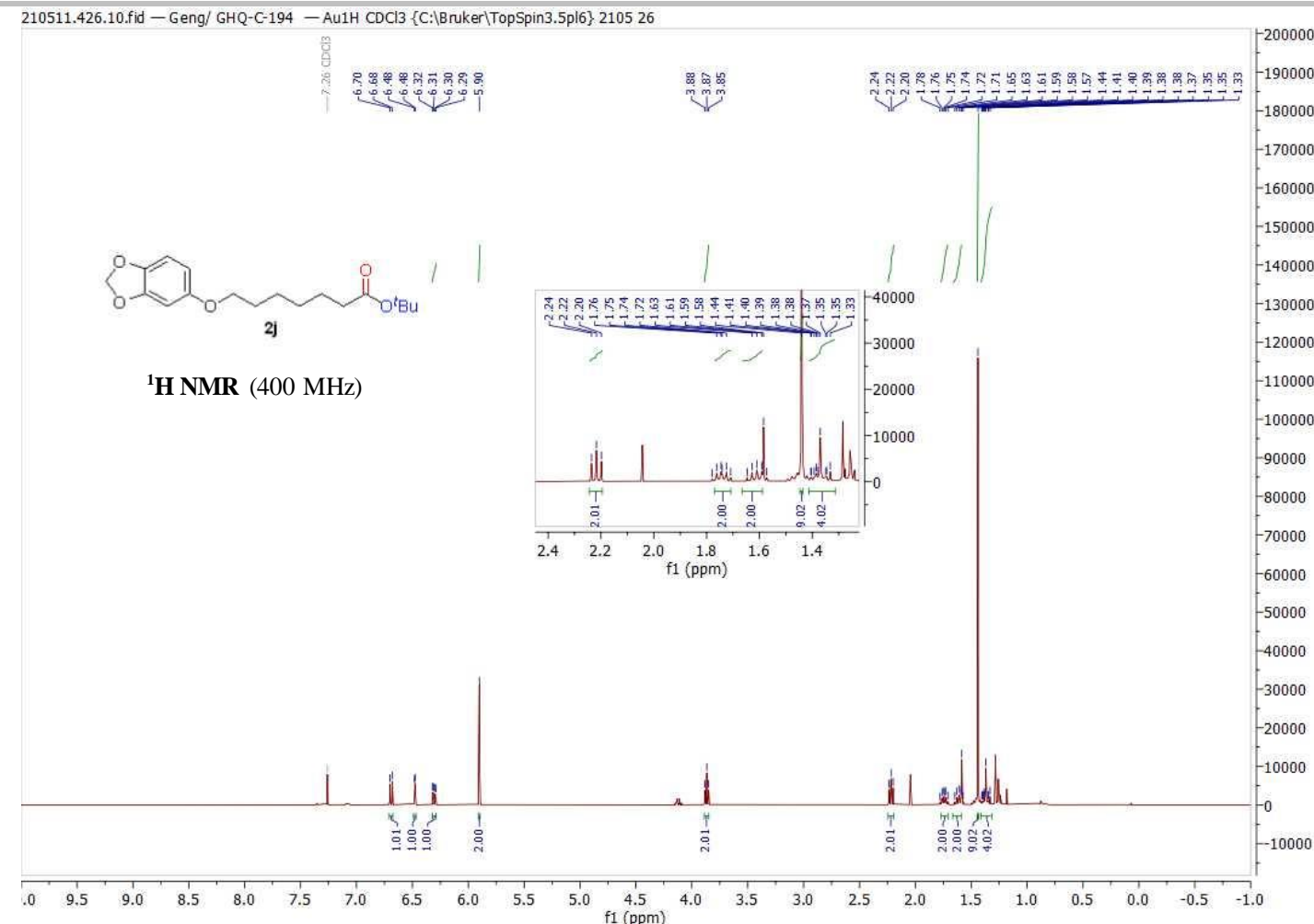

210511.426.11.fid - Geng/ GHQ-C-194-Au13C CDCl3 \{C:|Bruker|TopSpin3.5pl6\} 210526

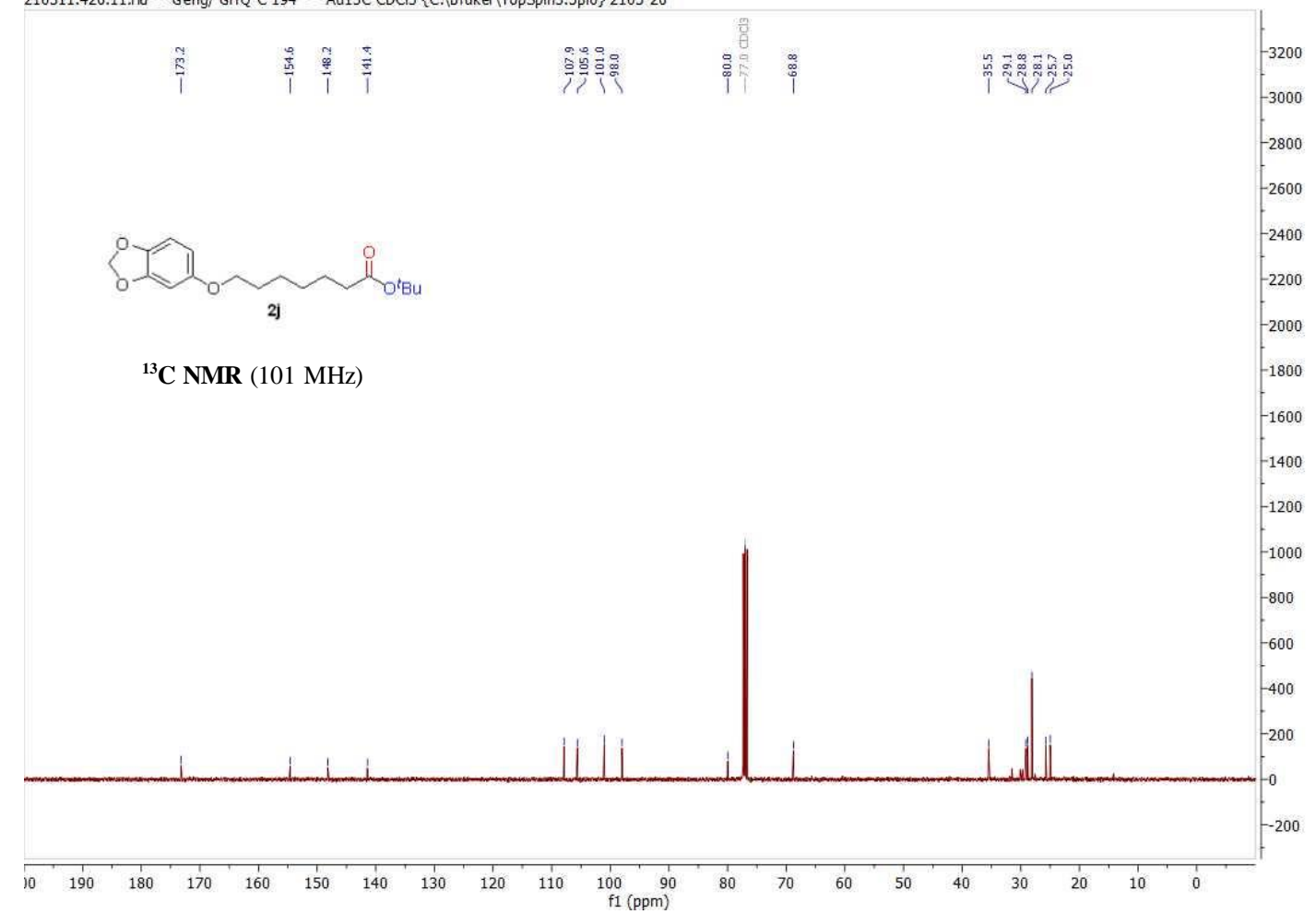


210426.461.10.fid - Huiqing Geng GHQ-C-154 - Au1H CDCl3 \{C:|Bruker|TopSpin3.5p|6\} 2104
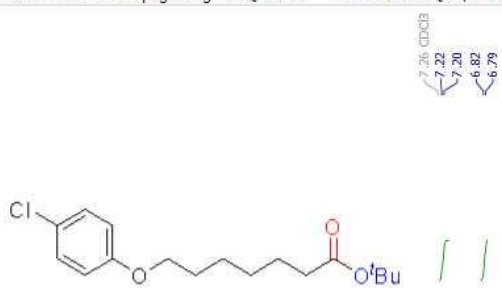

$2 \mathrm{k}$

${ }^{\mathbf{1}} \mathbf{H}$ NMR (400 MHz)
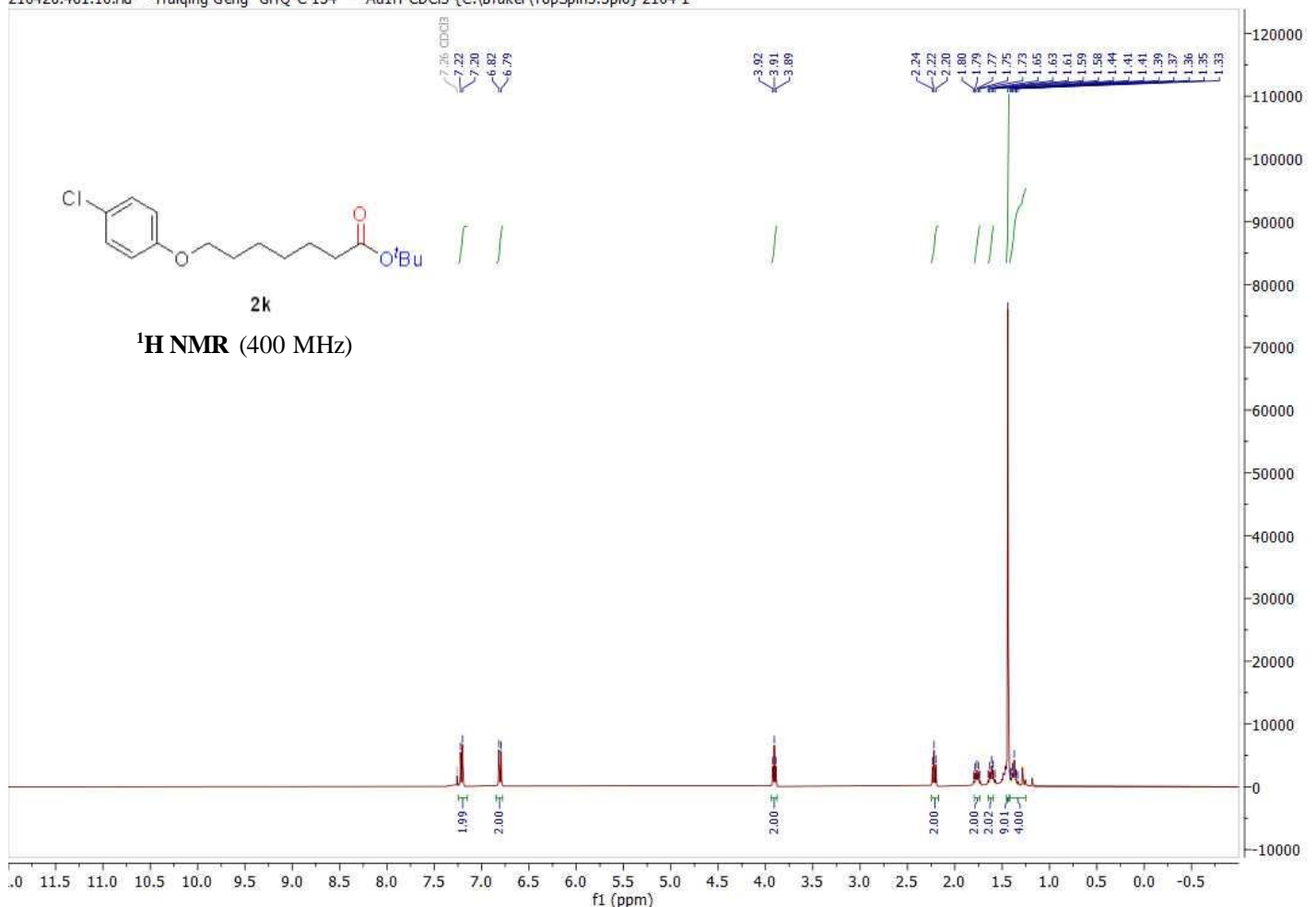

210426.461.11.fid - Huiqing Geng GHQ-C-154 - Au13C CDC13 \{C:|Bruker|TopSpin3.5pl6\} 2104

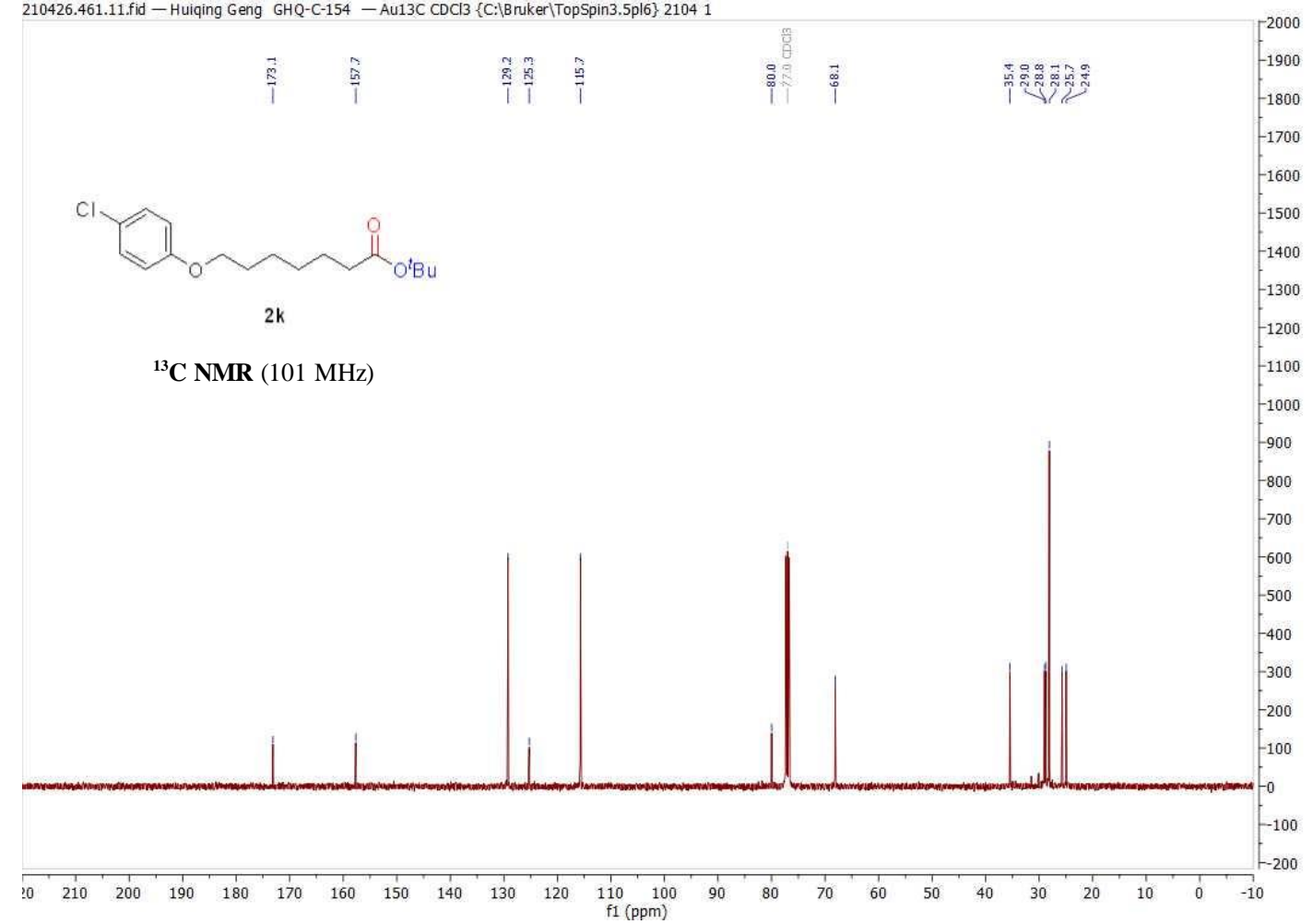


210517.440.10.fid - Huiqing Geng / GHQ-C-206 - Au1H CDCl3 \{C:|Bruker|TopSpin3.5pl6\} 210540

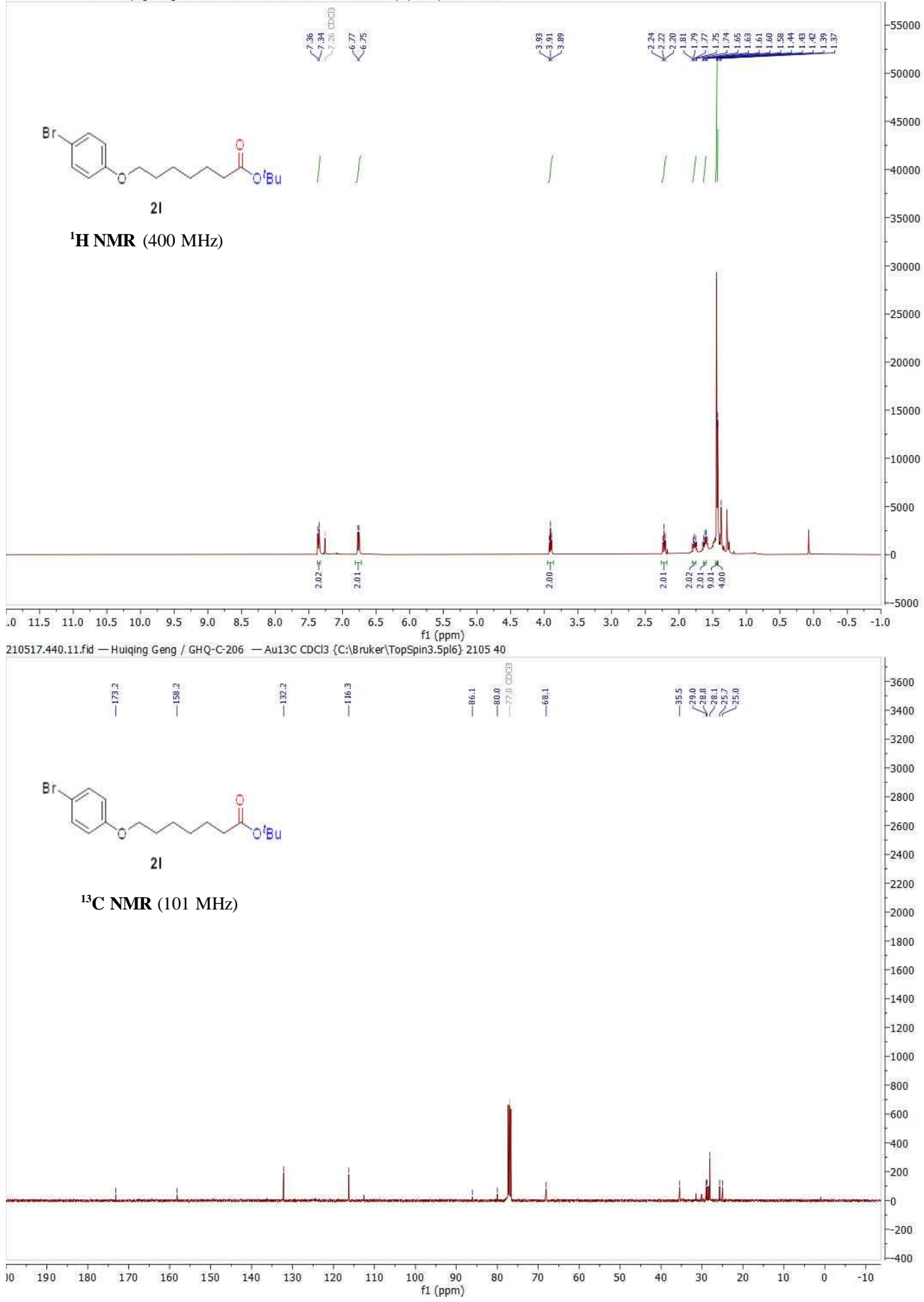


210511.307.10.fid - Geng/GHQ-CA-31 - Au1H CDCl3 \{C:|Bruker|TopSpin3.6.2\} 21057

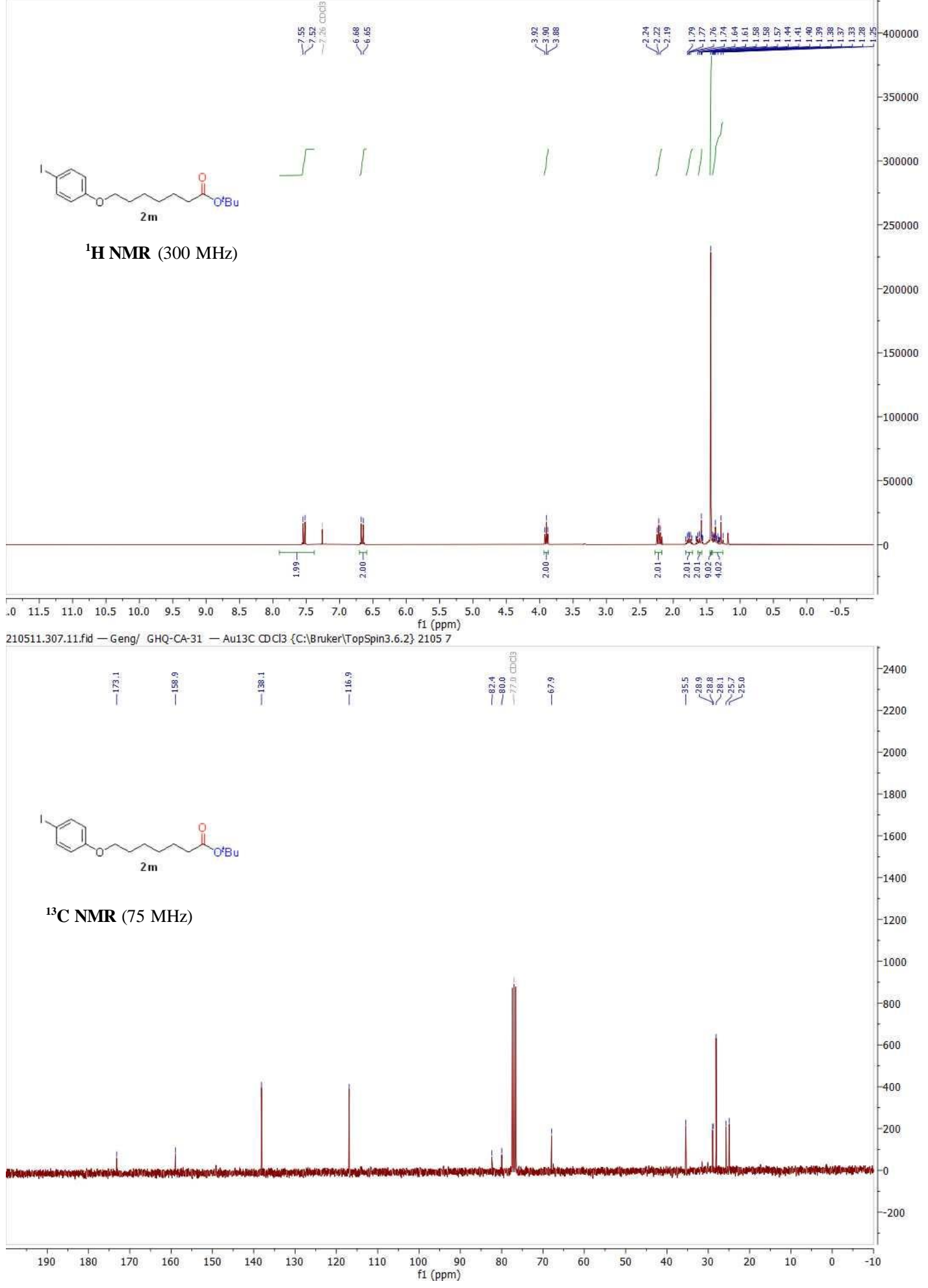



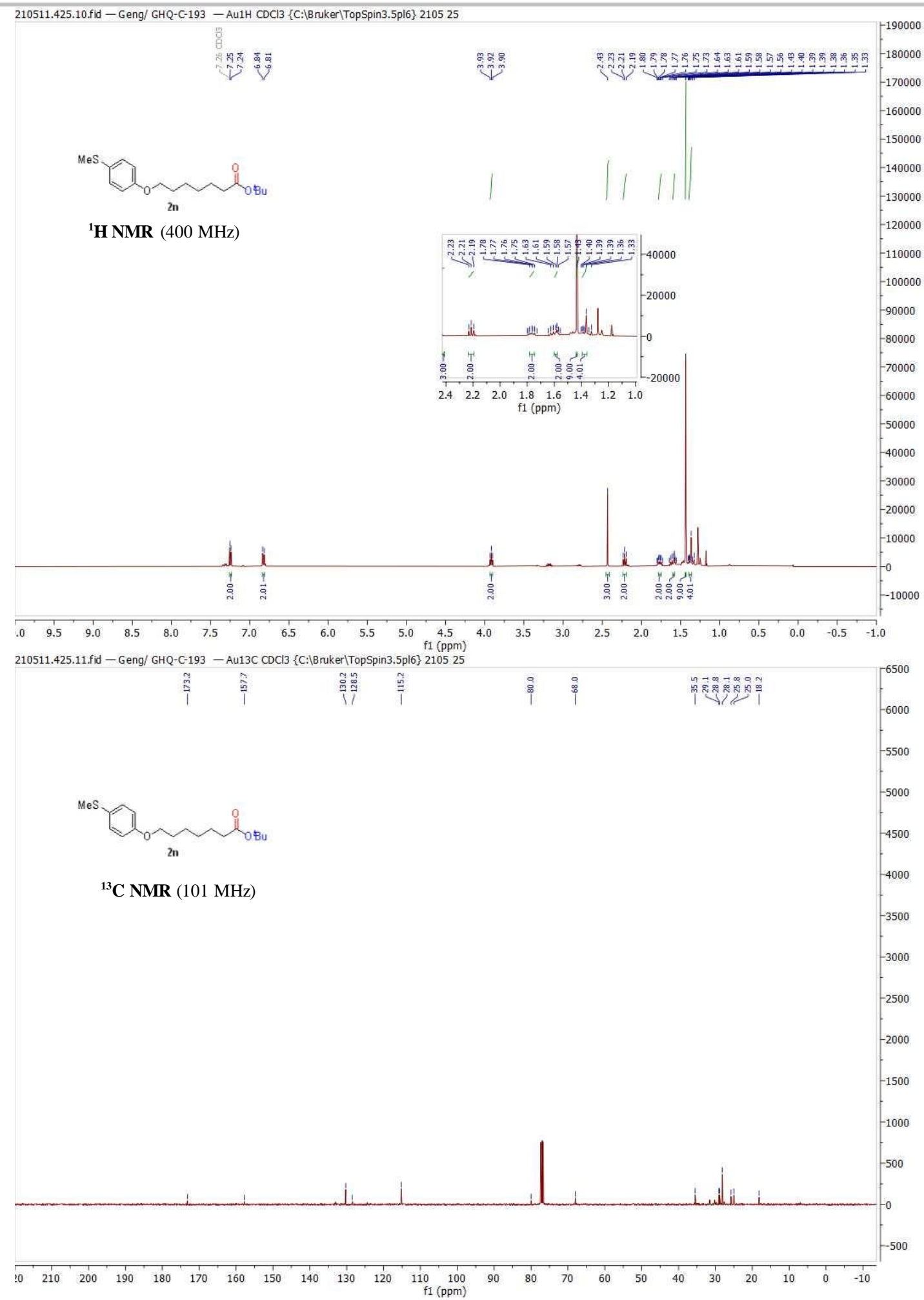


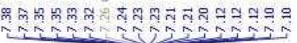<smiles>CCCCCCCCOc1cccc(C)c1</smiles>

20

${ }^{1}$ H NMR (300 MHz)

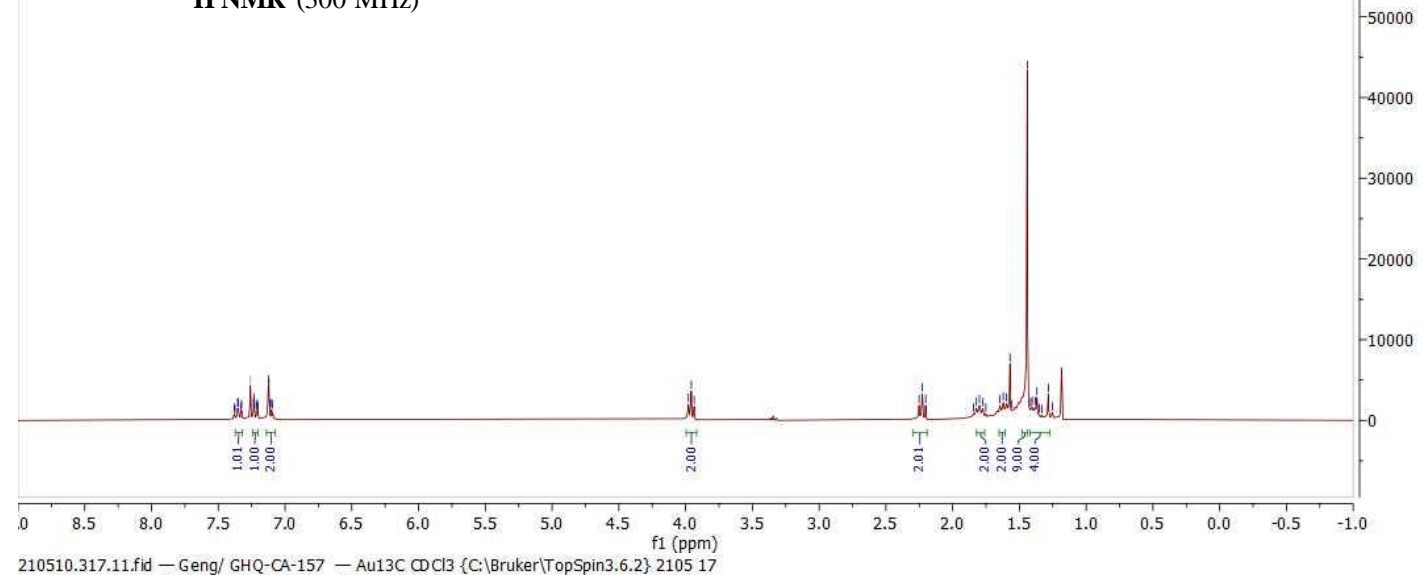

210510.317.11.fid - Geng/ GHQ-CA-157 - Au13C CDCl3 \{C:|Bruker|TopSpin3.6.2\} 2105 17

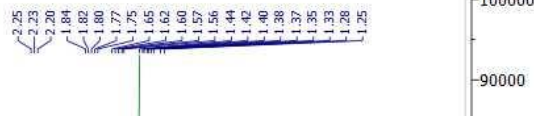

$-80000$

$-100000$

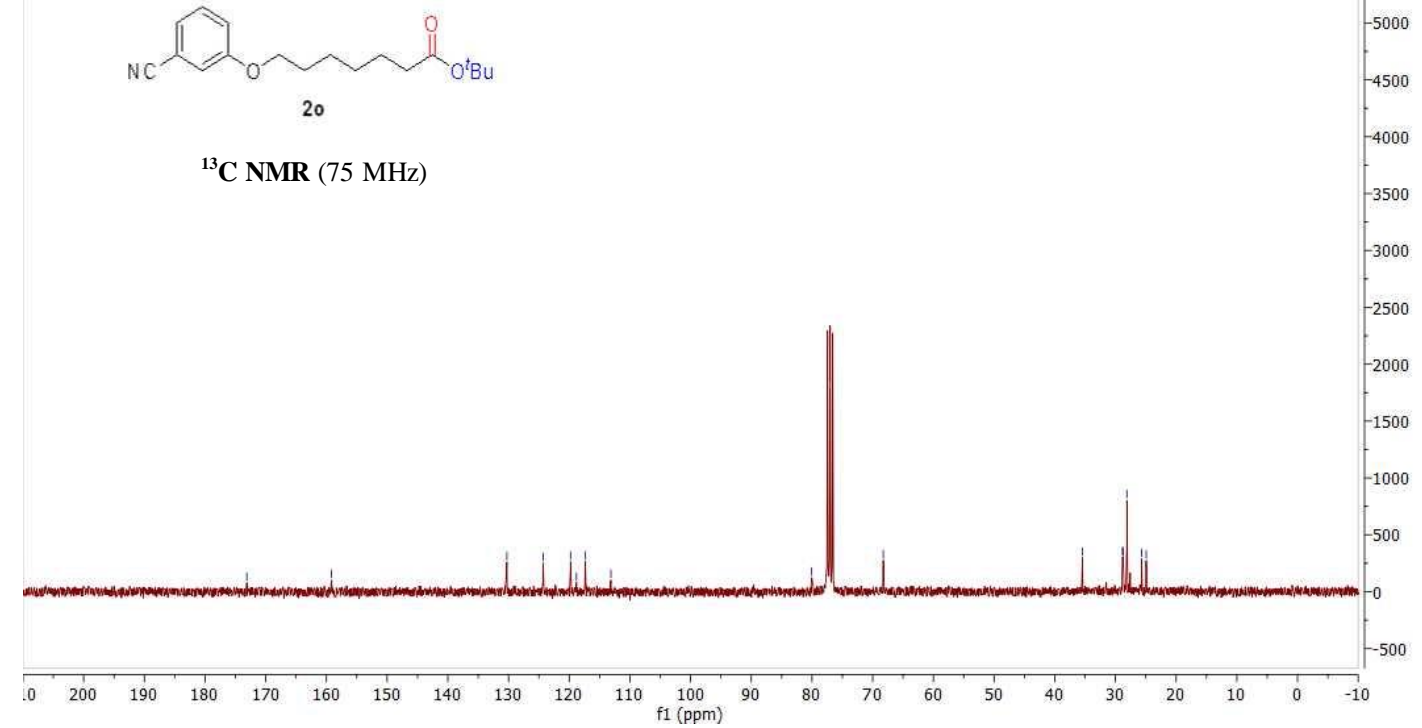

20

${ }^{13}$ C NMR (75 MHz) 


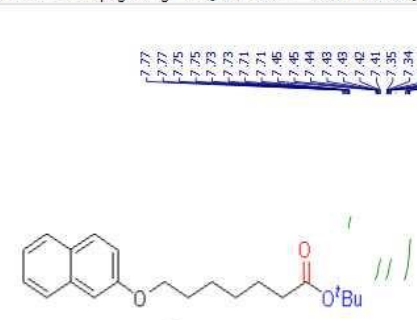

$2 \mathrm{p}$

${ }^{1}$ H NMR (400 MHz)

\section{送誉}

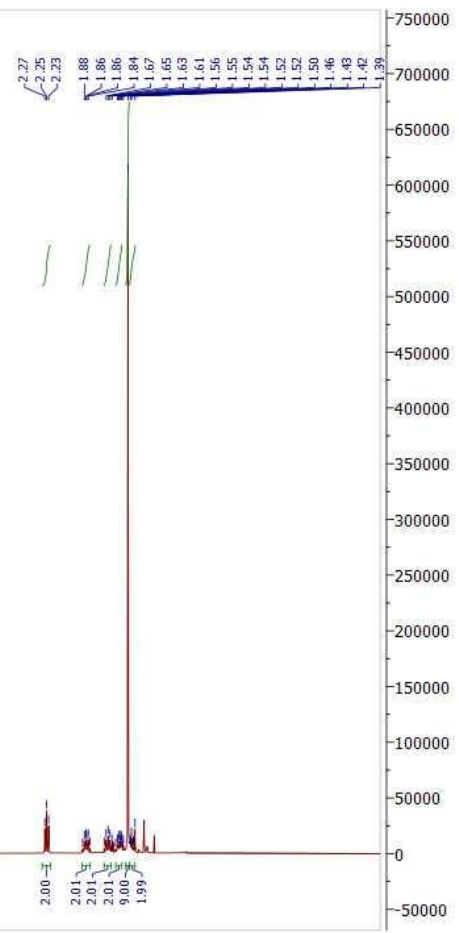

$-50000$ 210426.463.11.fid - Huiqing Geng GHQ-C-155-2 - Au13C CDCl3 \{C:|Bruker|TopSpin3.5pl6\} 21043

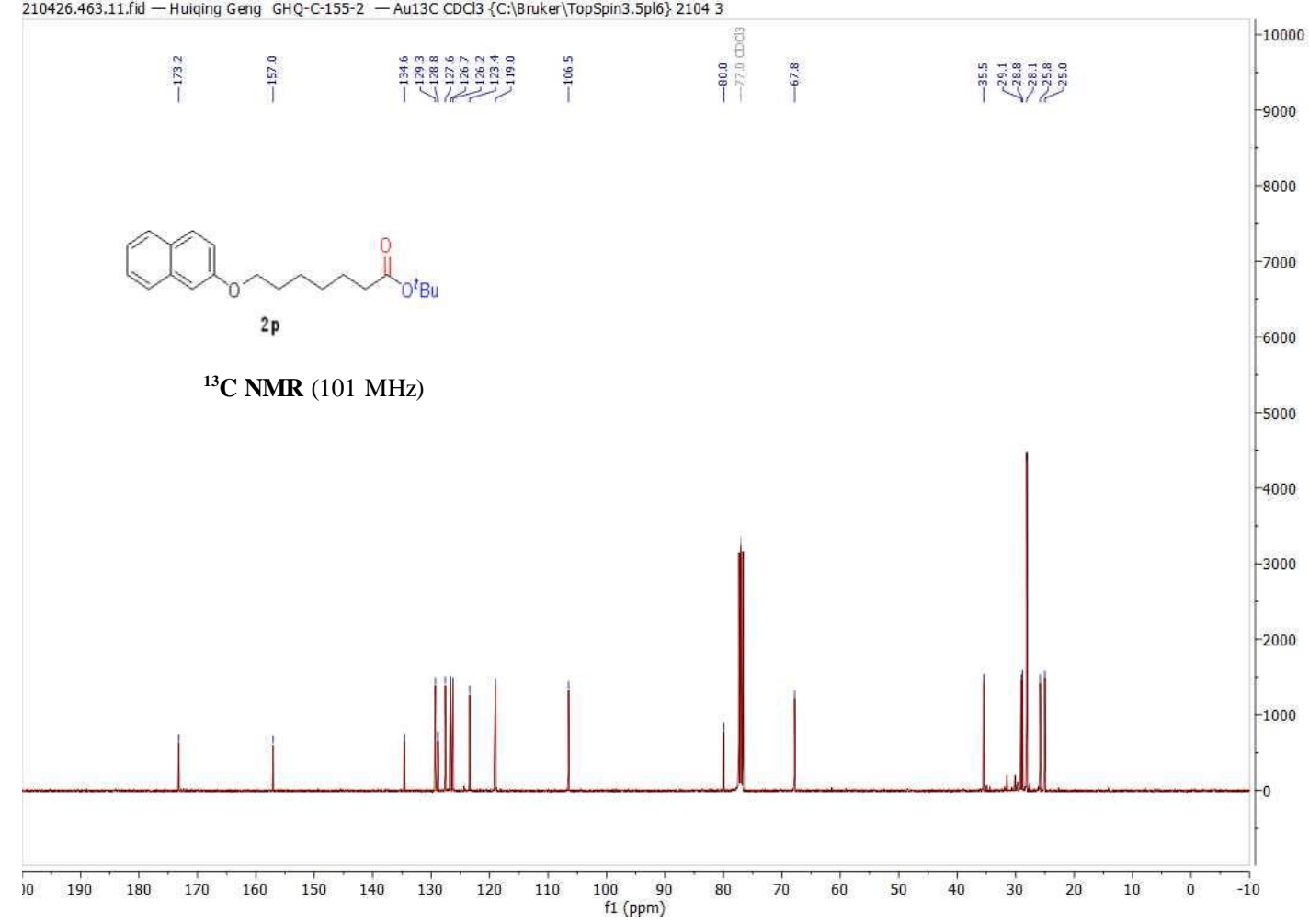



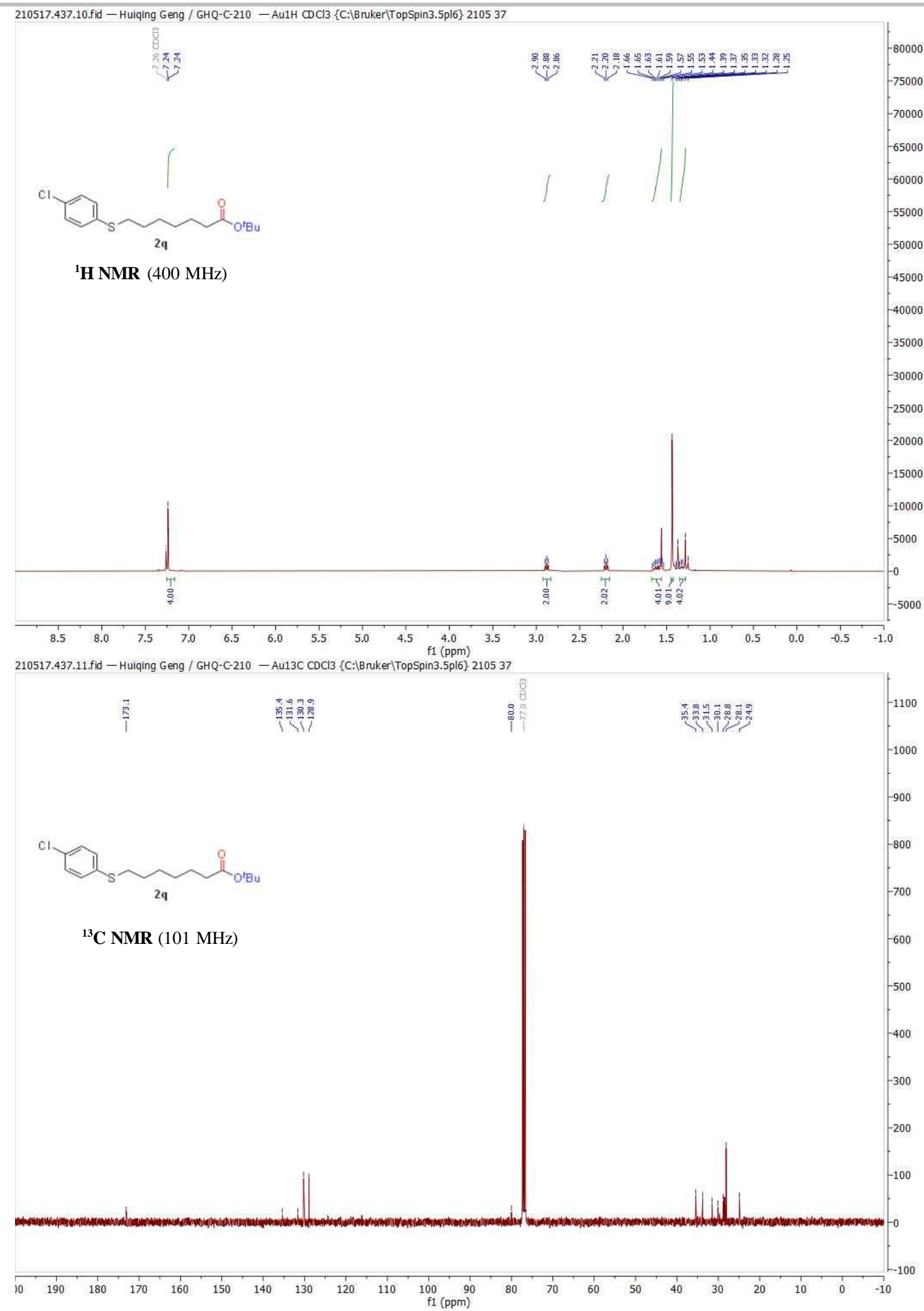


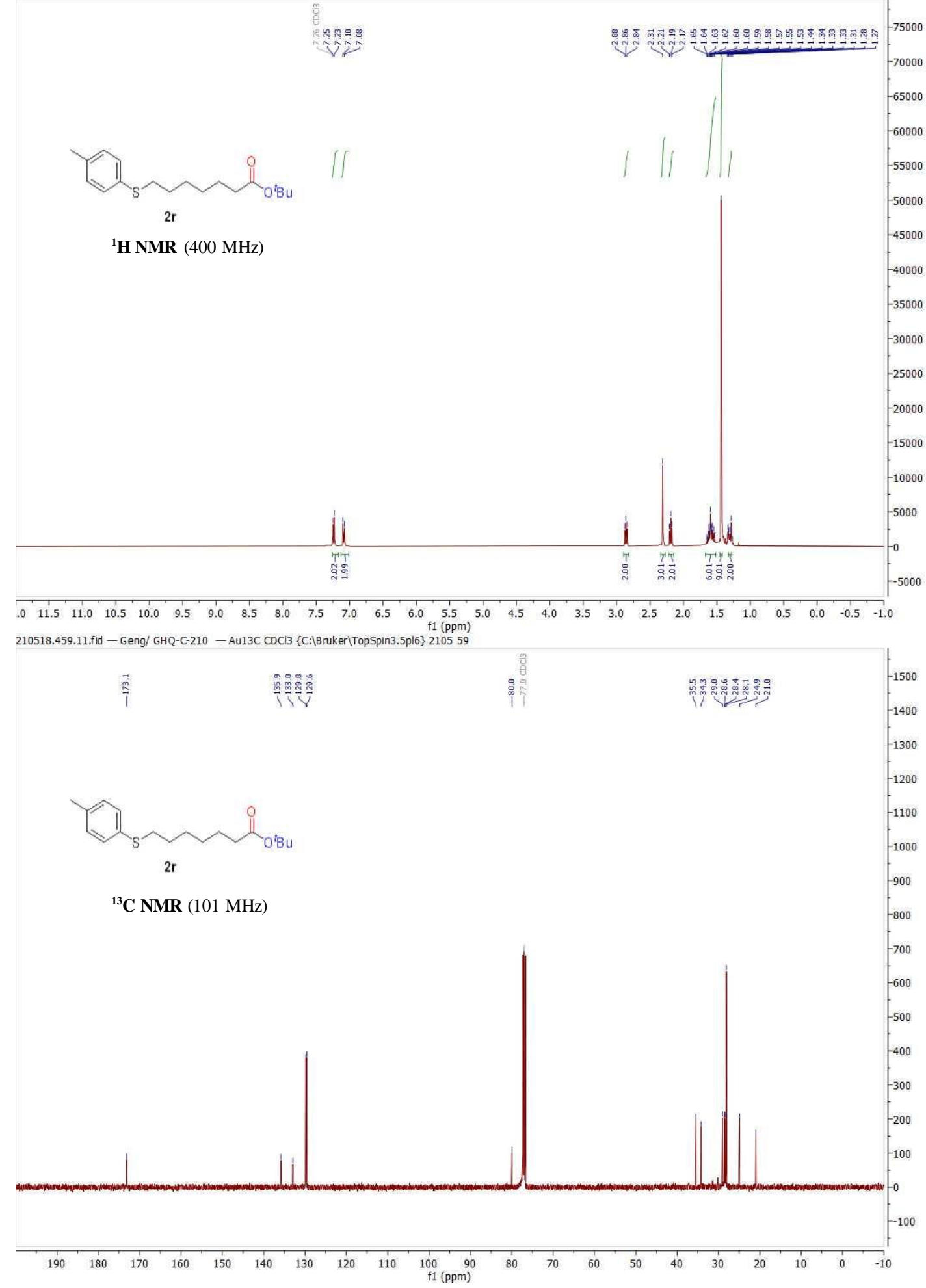


210202.f326.10.fid - Huiqing Geng GHQ-C-98 - PROTON CDCl3 \{C:|Bruker|TopSpin3.6.2\} 210226

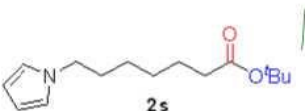

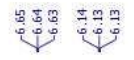

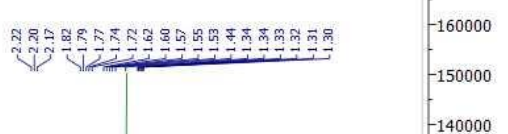

${ }^{1}$ H NMR $(300 \mathrm{MHz})$

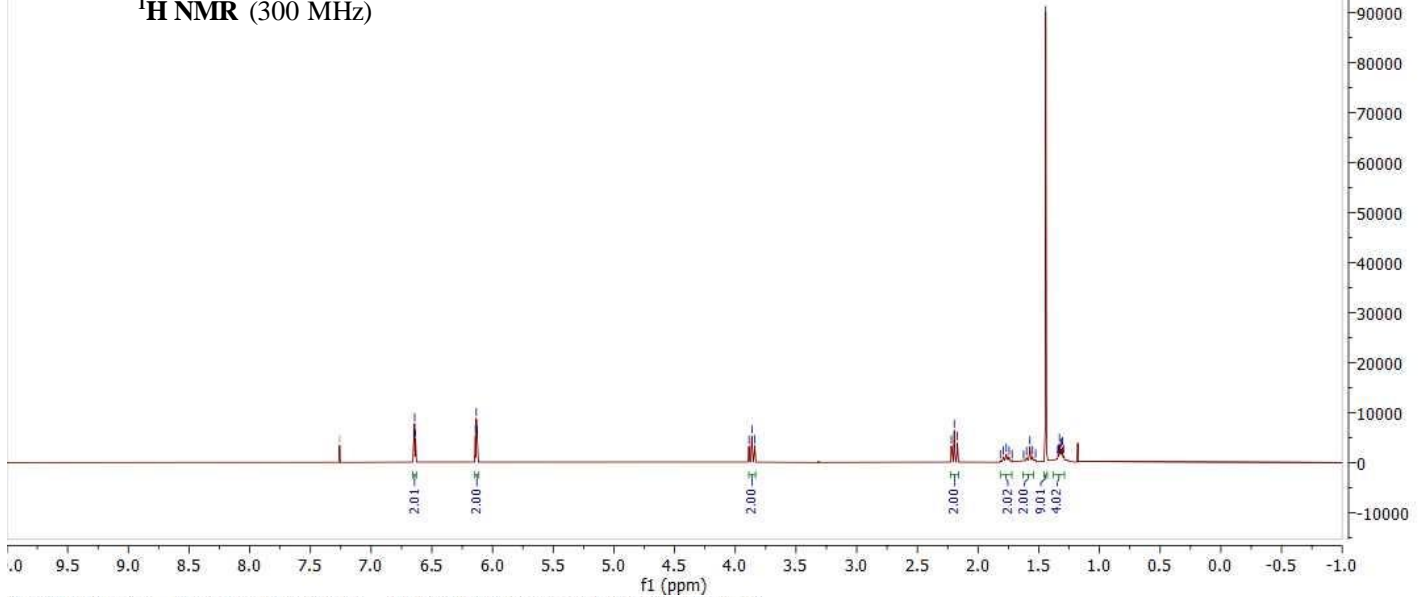

210202.f326.11.fid - Huiqing Geng GHQ-C-98 - C13CPD CDCl3 \{C:|Bruker|TopSpin3.6.2\} 210226

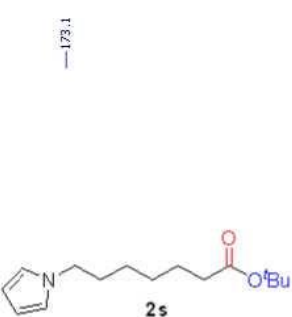

${ }^{13}$ C NMR (75 MHz)

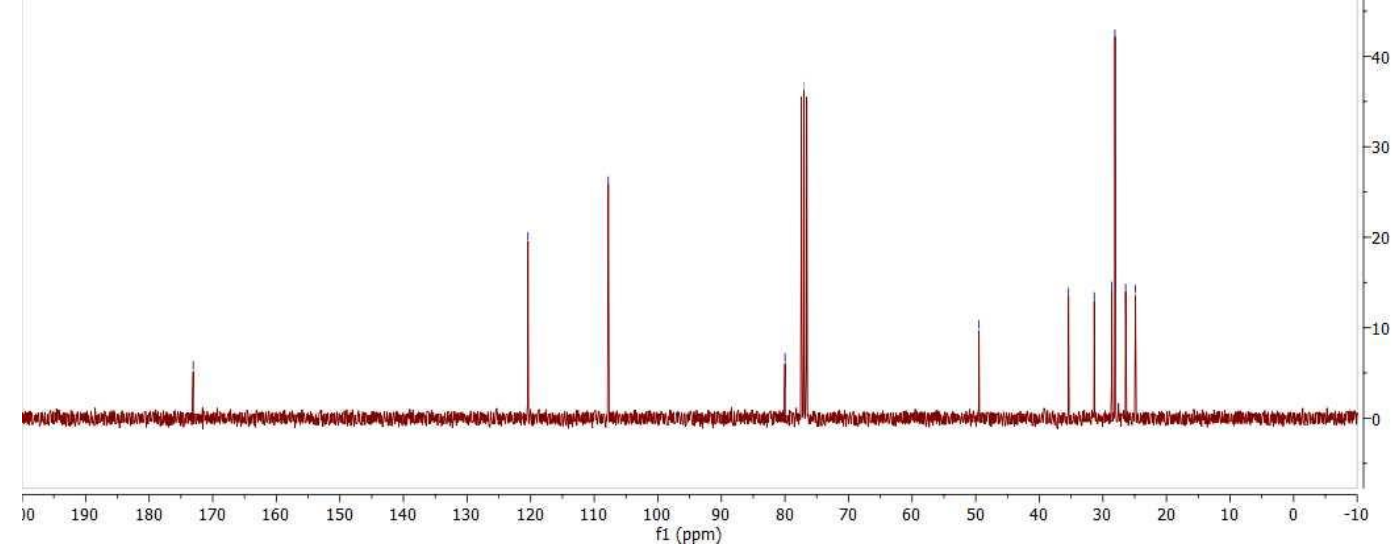



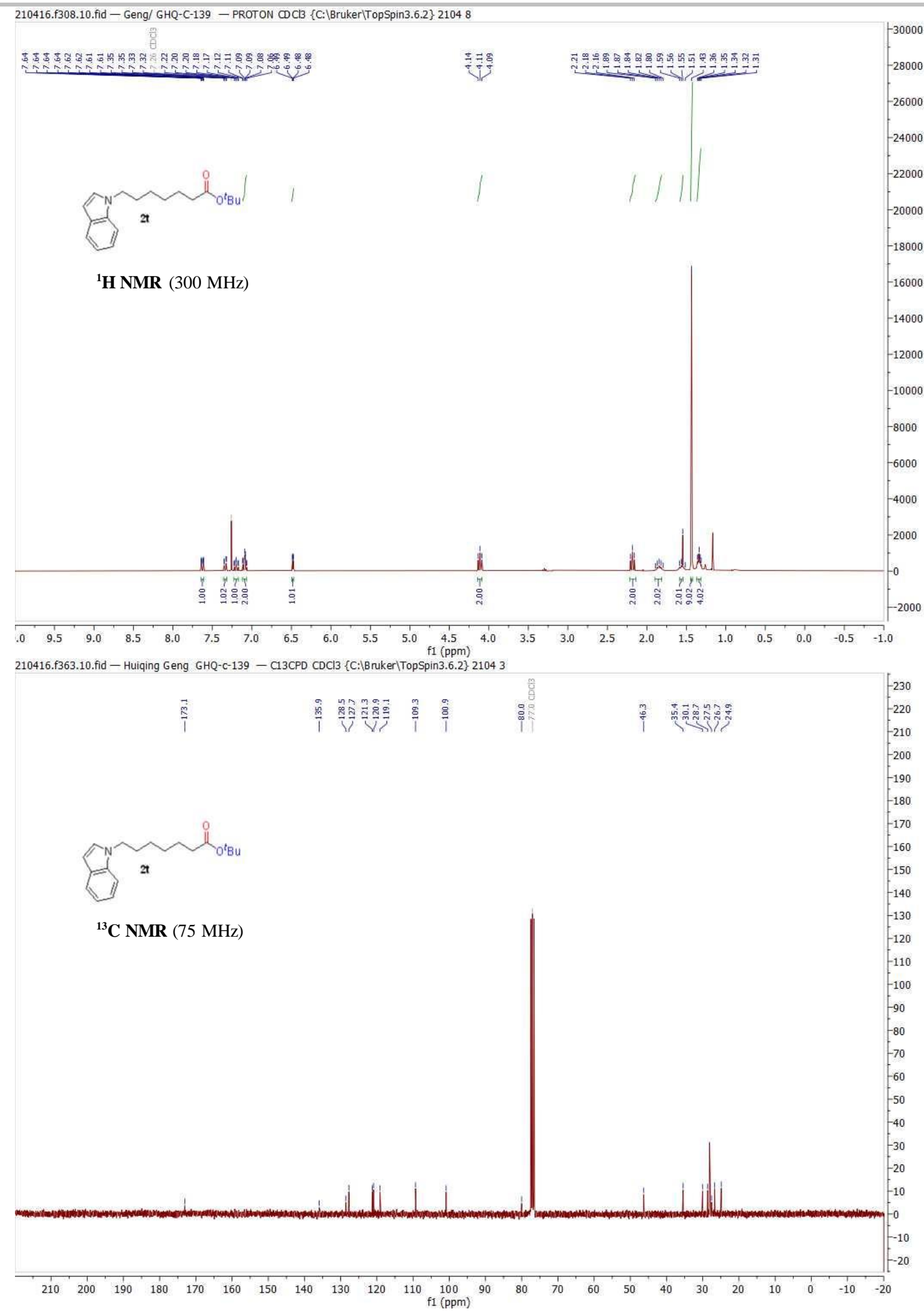

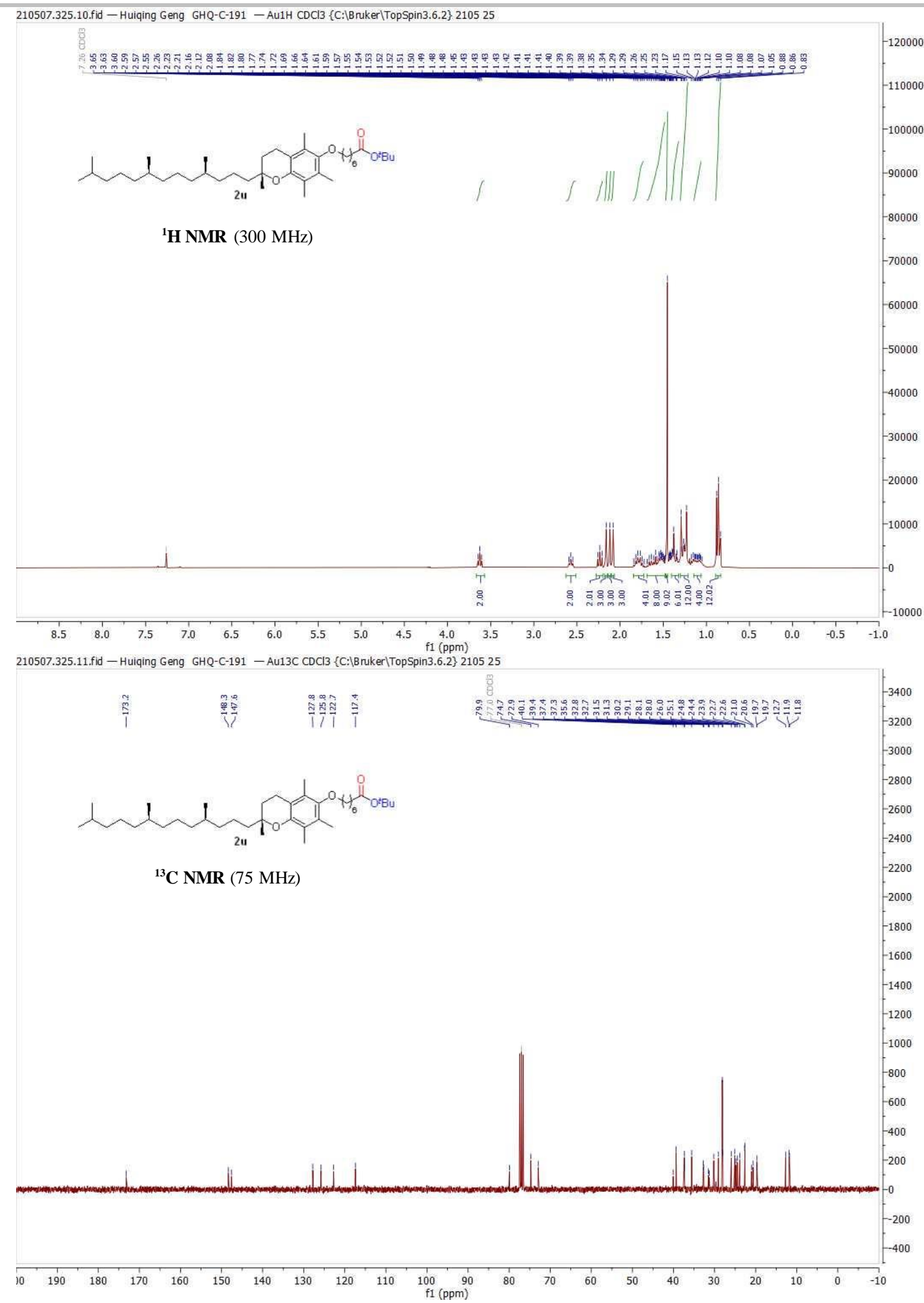


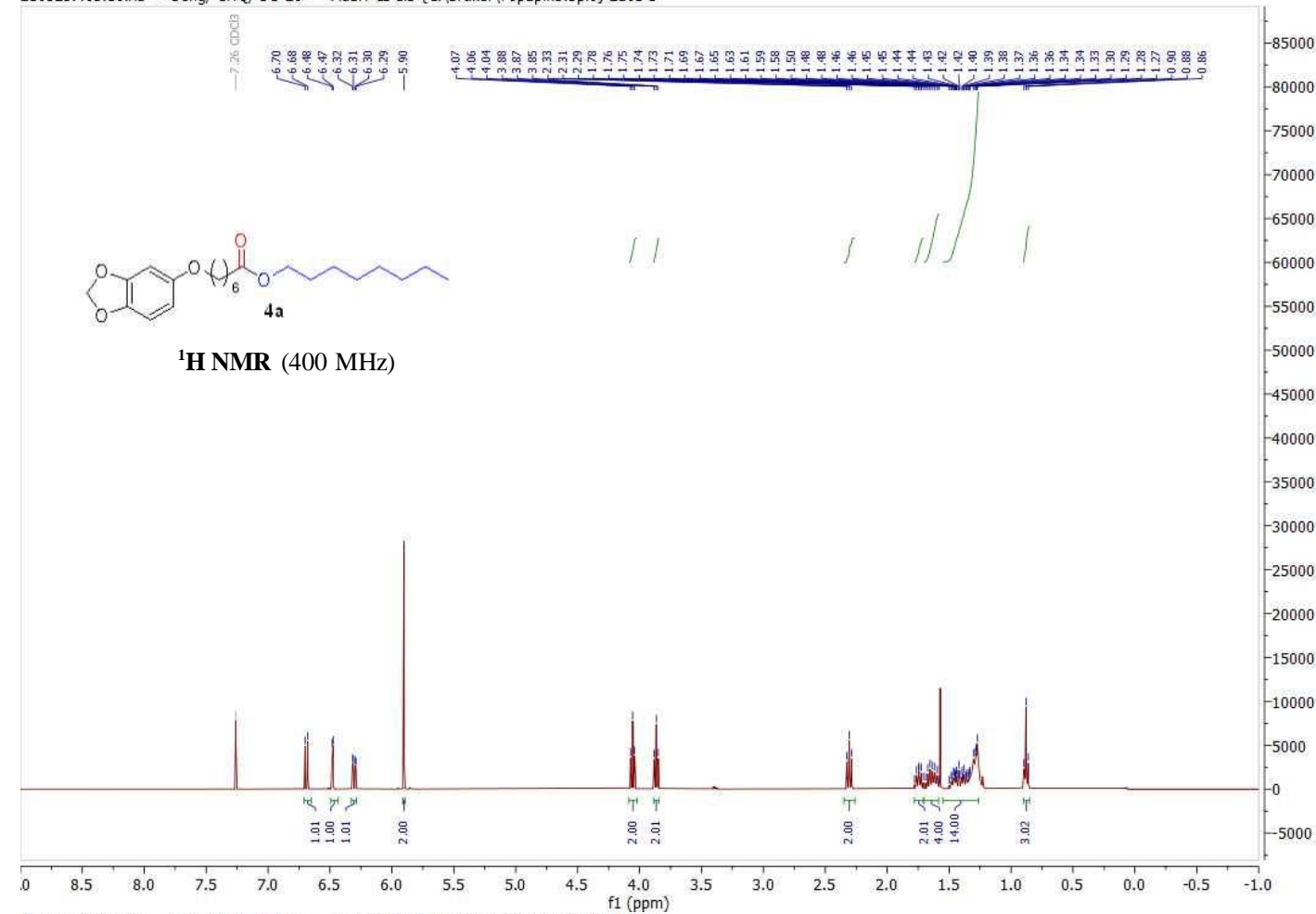

210525.403.11.fid - Geng/ GHQ/ DB-20 - Au13C CDCl3 \{C:|Bruker|TopSpin3.5pl6\} 21053

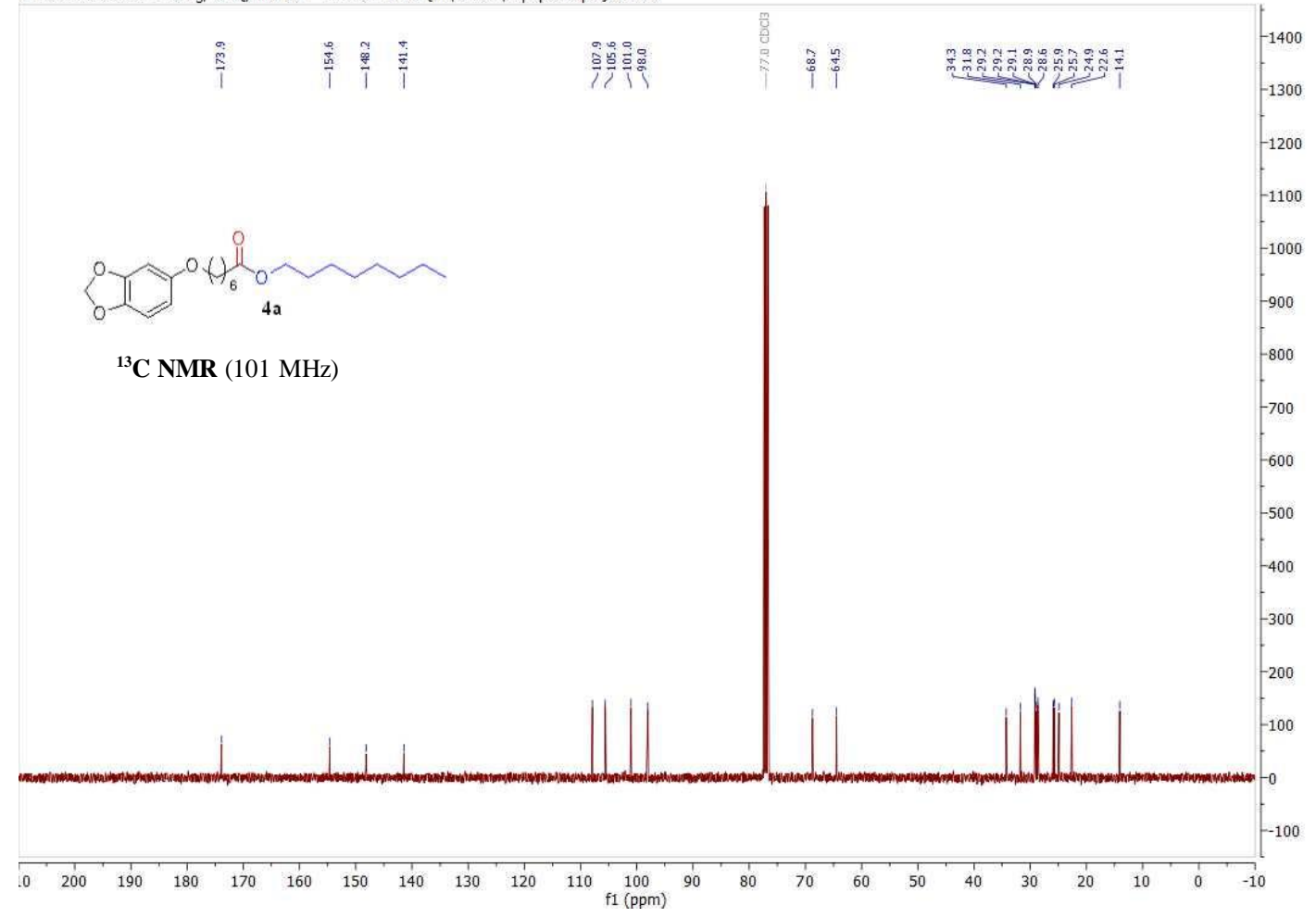




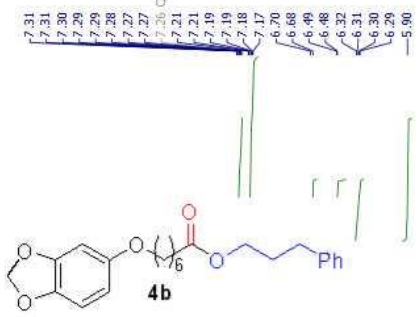

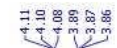

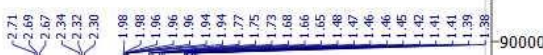

${ }^{1}$ H NMR (400 MHz)

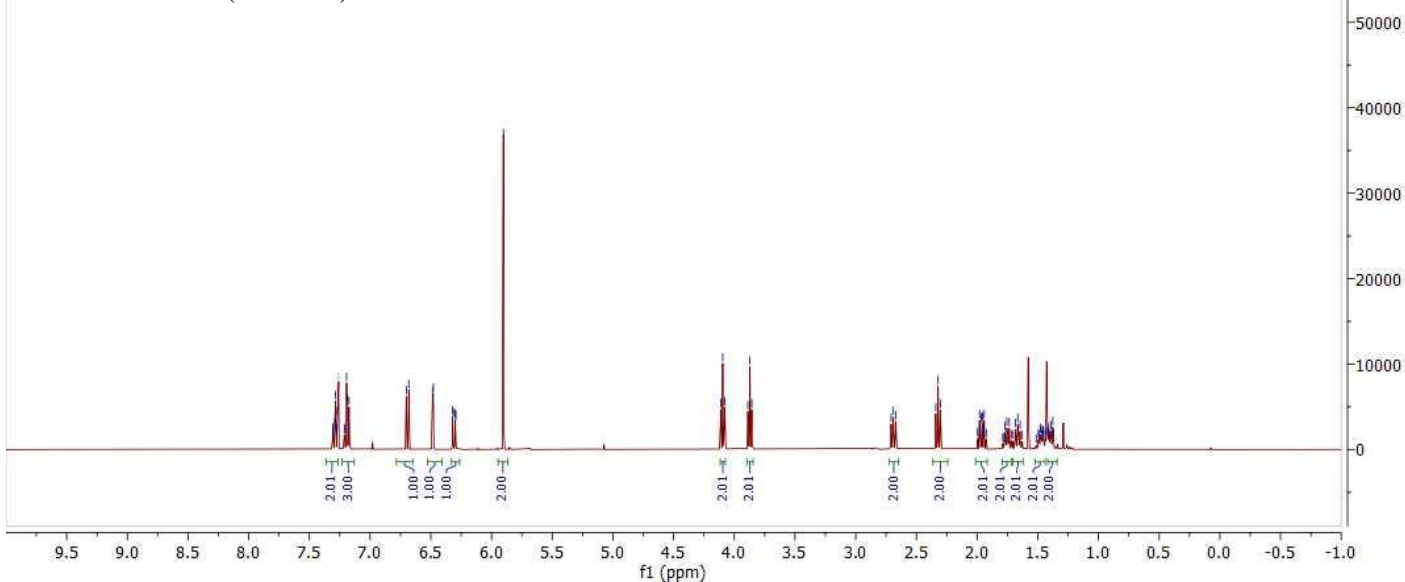

210525.404.11.fid - Geng/ GHQ/ DB-21 - Au13C CDCl3 \{C:|Bruker|TopSpin3.5pl6\} 2105 4

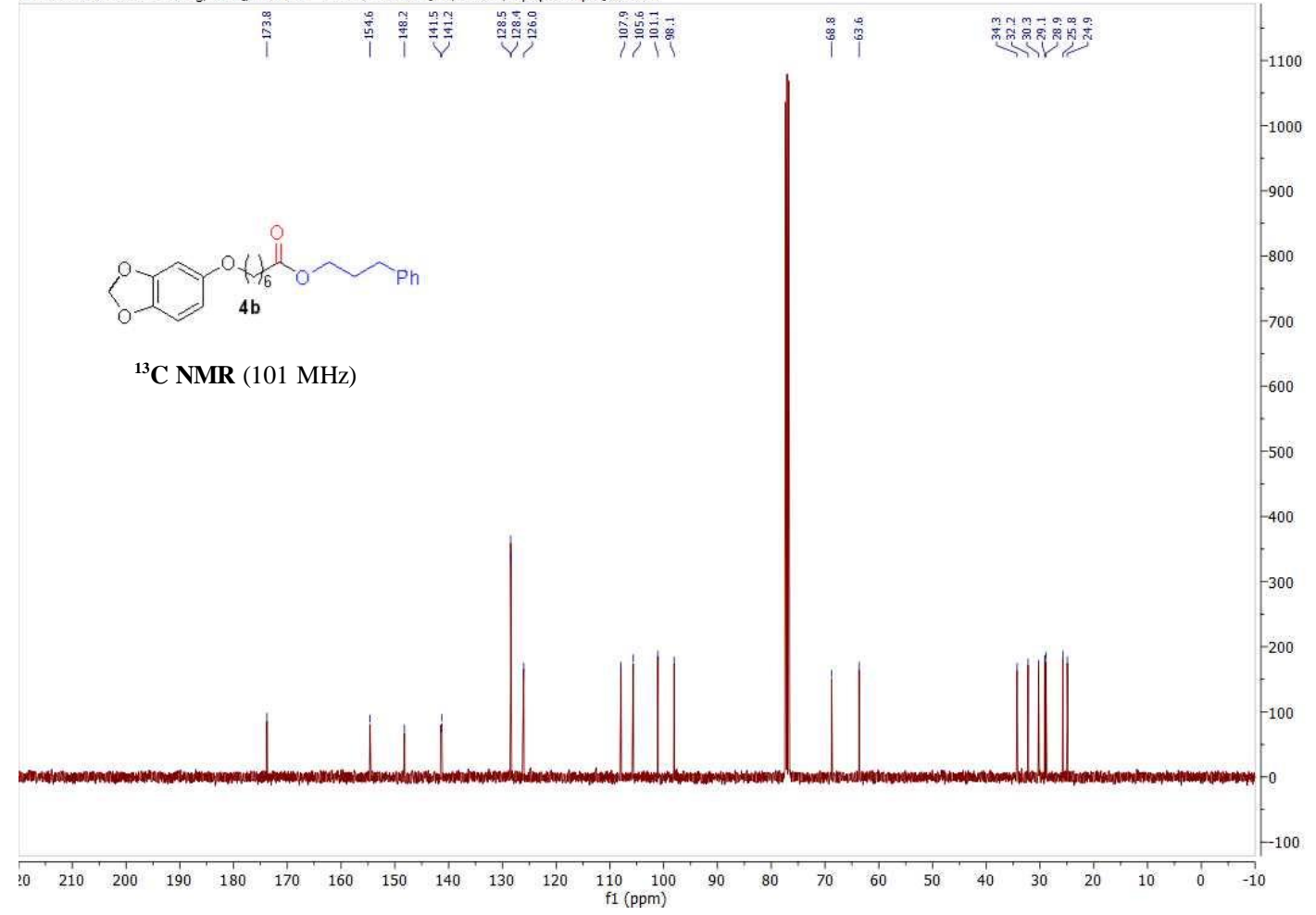




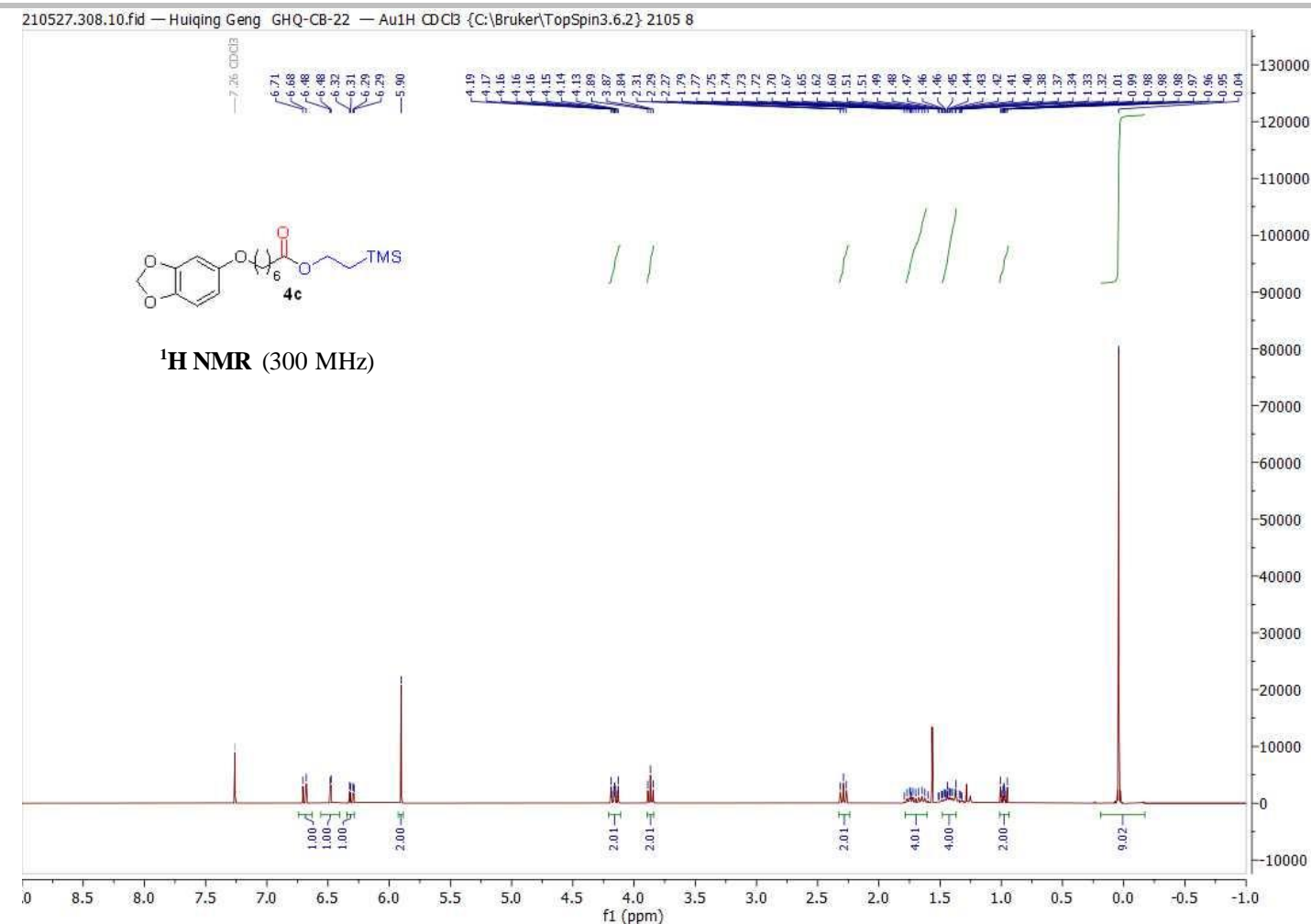

210527.308.11.fid - Huiqing Geng GHQ-CB-22 - Au13C CDCl3 \{C:|Bruker|TopSpin3.6.2\} 21058

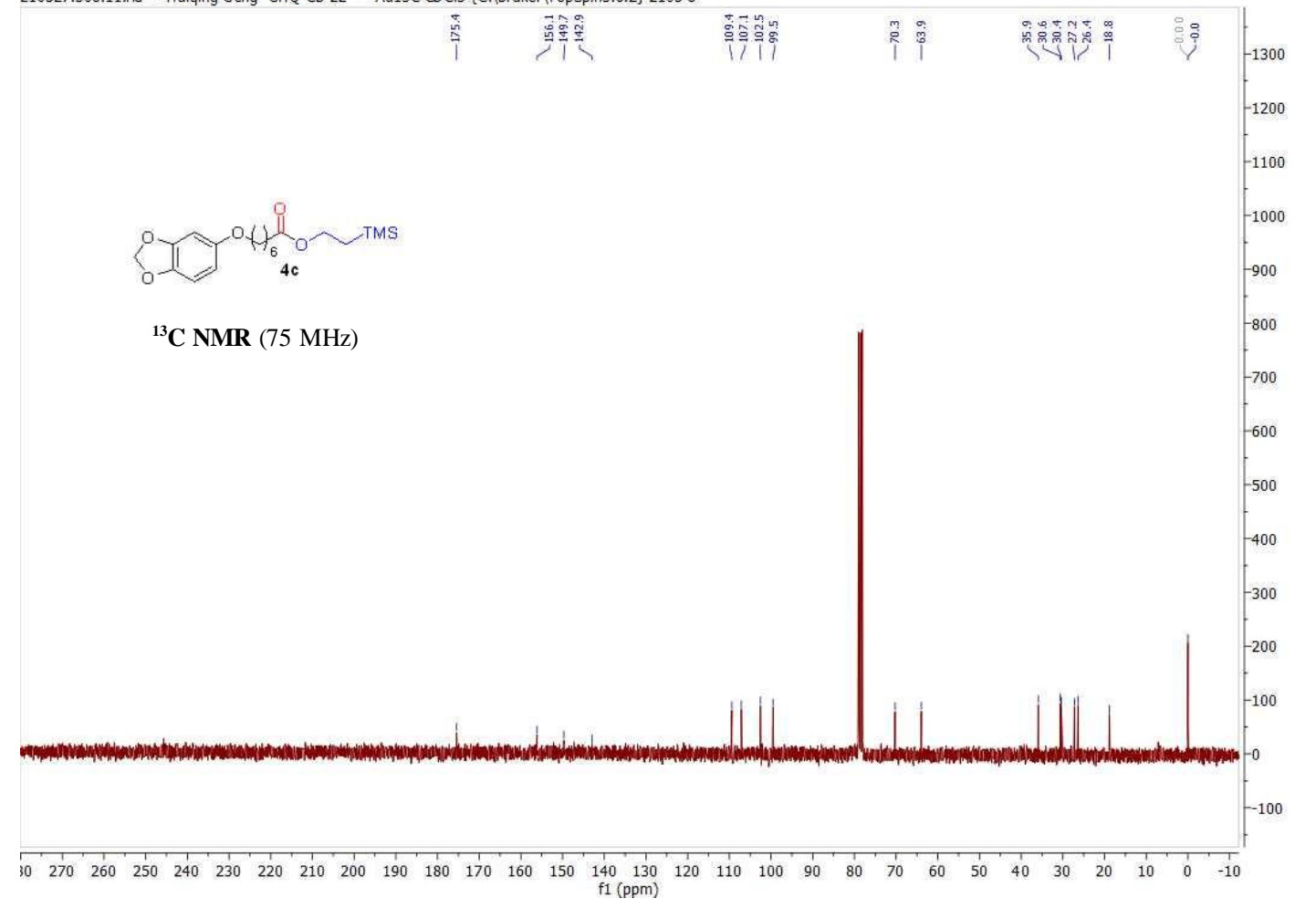



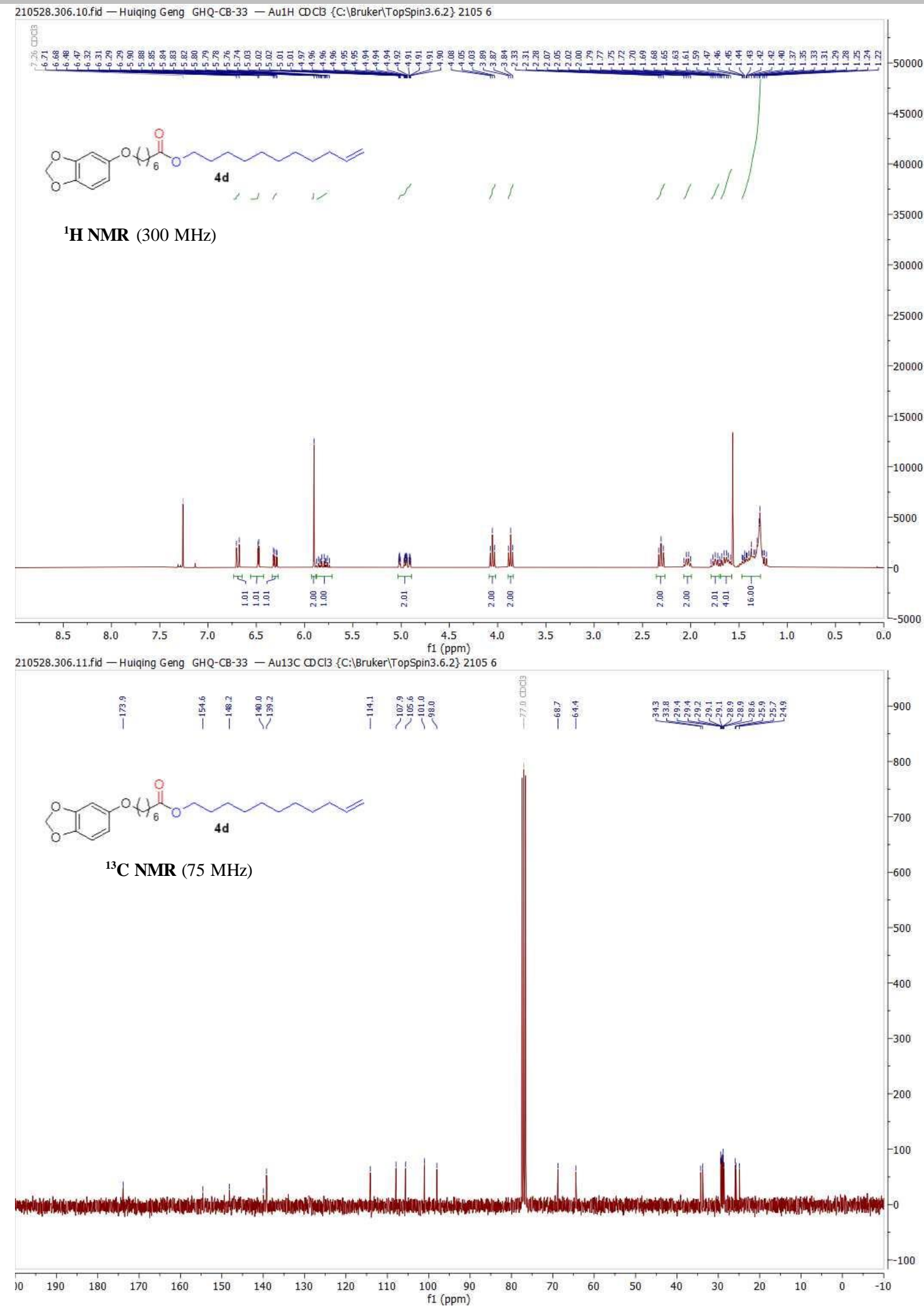
${ }^{1} \mathbf{H}$ NMR $(400 \mathrm{MHz})$

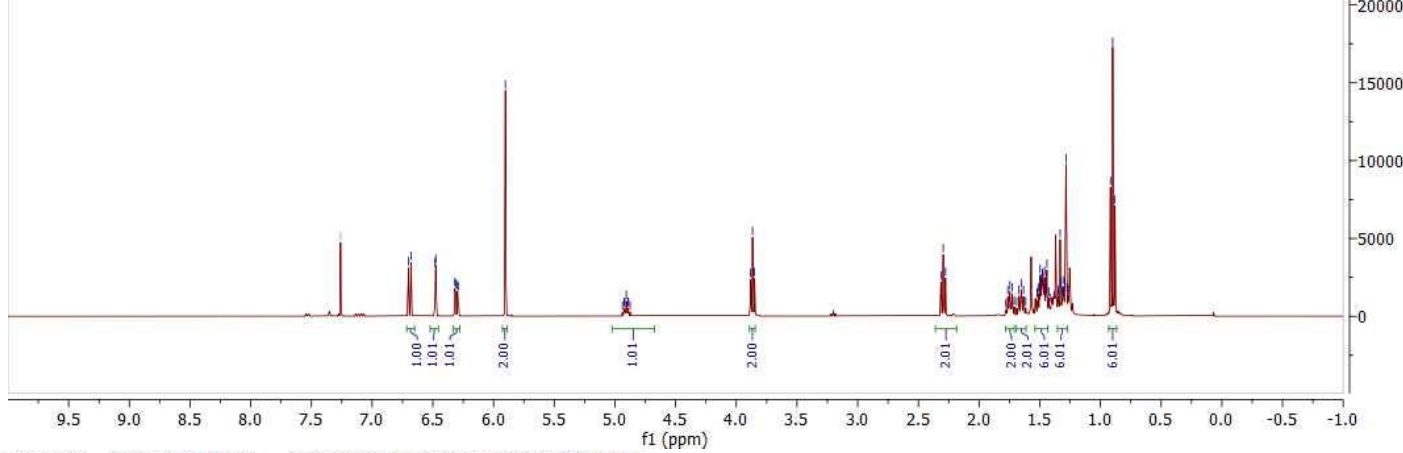

210525.405.11.fid - Geng/ GHQ/ DB-24 - Au13C CDCl3 \{C:|Bruker|TopSpin3.5pl6\} 21055

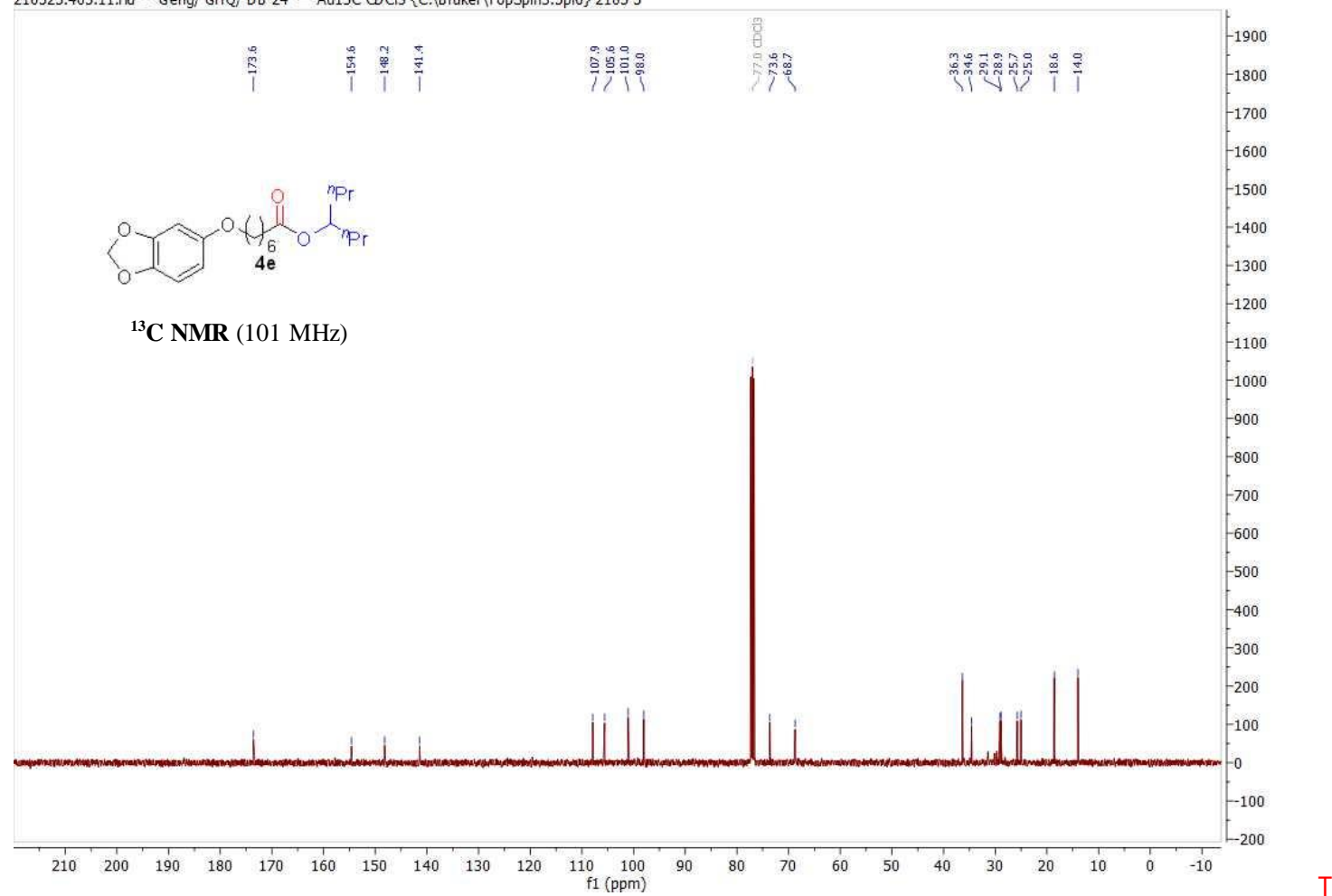

Thanks! 


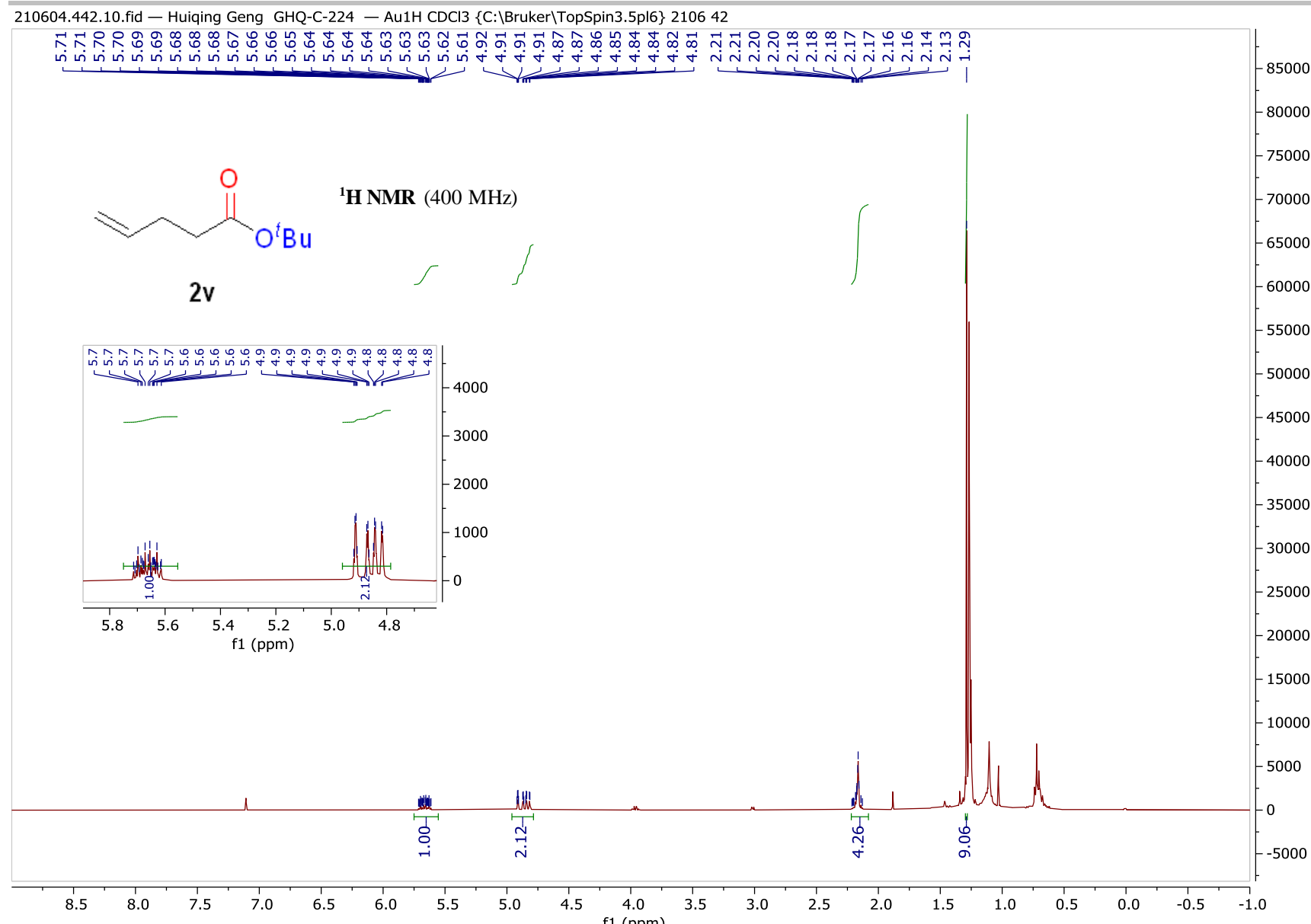

210604.442.11.fid - Huiqing Geng GHQ-C-224 - Au13C CDCl3 \{C:|Bruker|TopSpin3.5pl6\} 210642

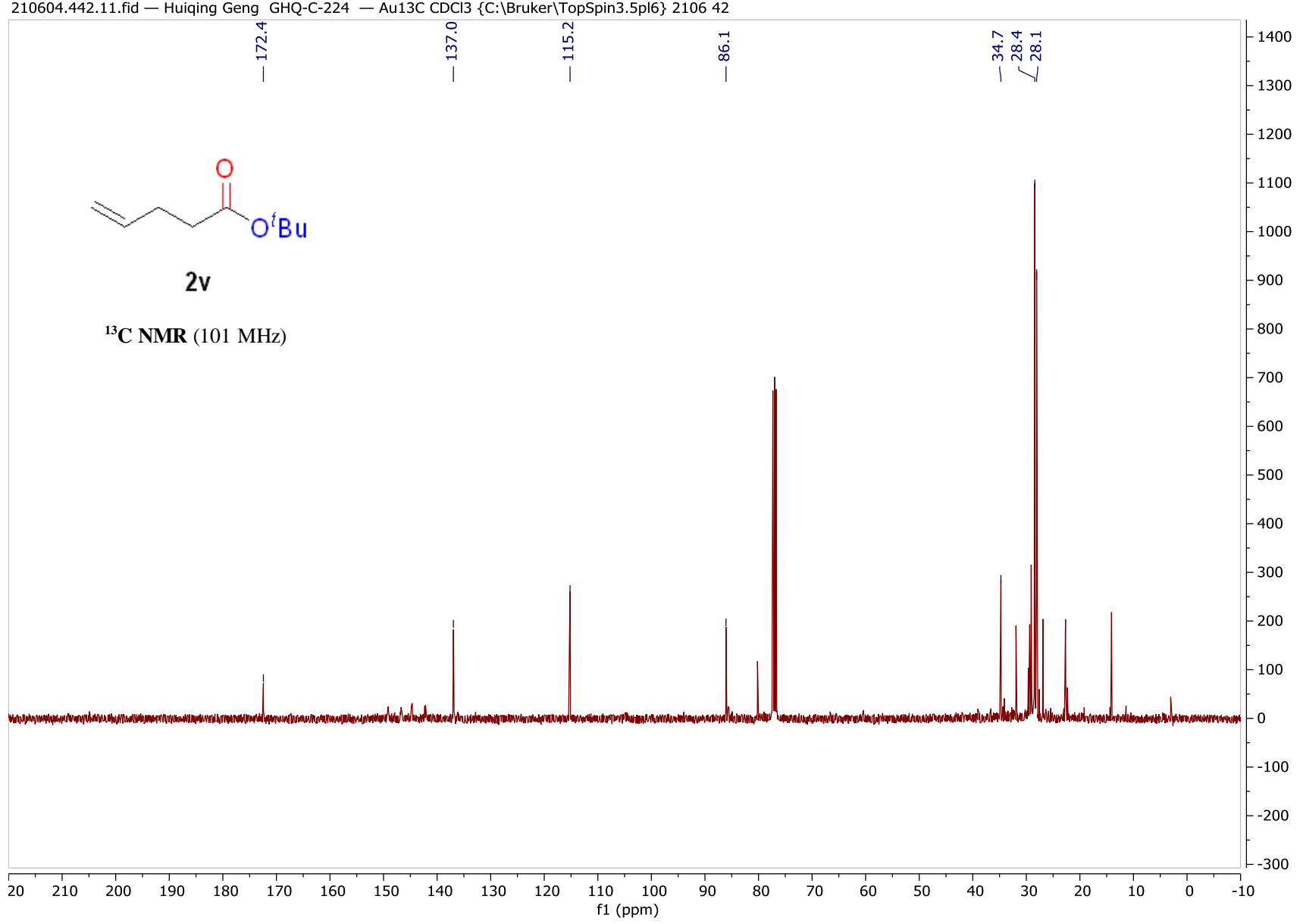

